\title{
Creative Assets and Program Content Guide: To Build Social and Emotional Learning and Promote Trauma Mitigation and Healing
}

Adolescent Girl and Creativity Network

Follow this and additional works at: https://knowledgecommons.popcouncil.org/departments_sbsr-pgy

Part of the Community-Based Learning Commons, Family, Life Course, and Society Commons, Gender and Sexuality Commons, and the International Public Health Commons How does access to this work benefit you? Let us know!

\section{Recommended Citation}

Adolescent Girl and Creativity Network. 2020. "Creative Assets and Program Content Guide: To Build Social and Emotional Learning and Promote Trauma Mitigation and Healing," Christina Mallie and Sophie Soares (eds.). New York: Population Council. 


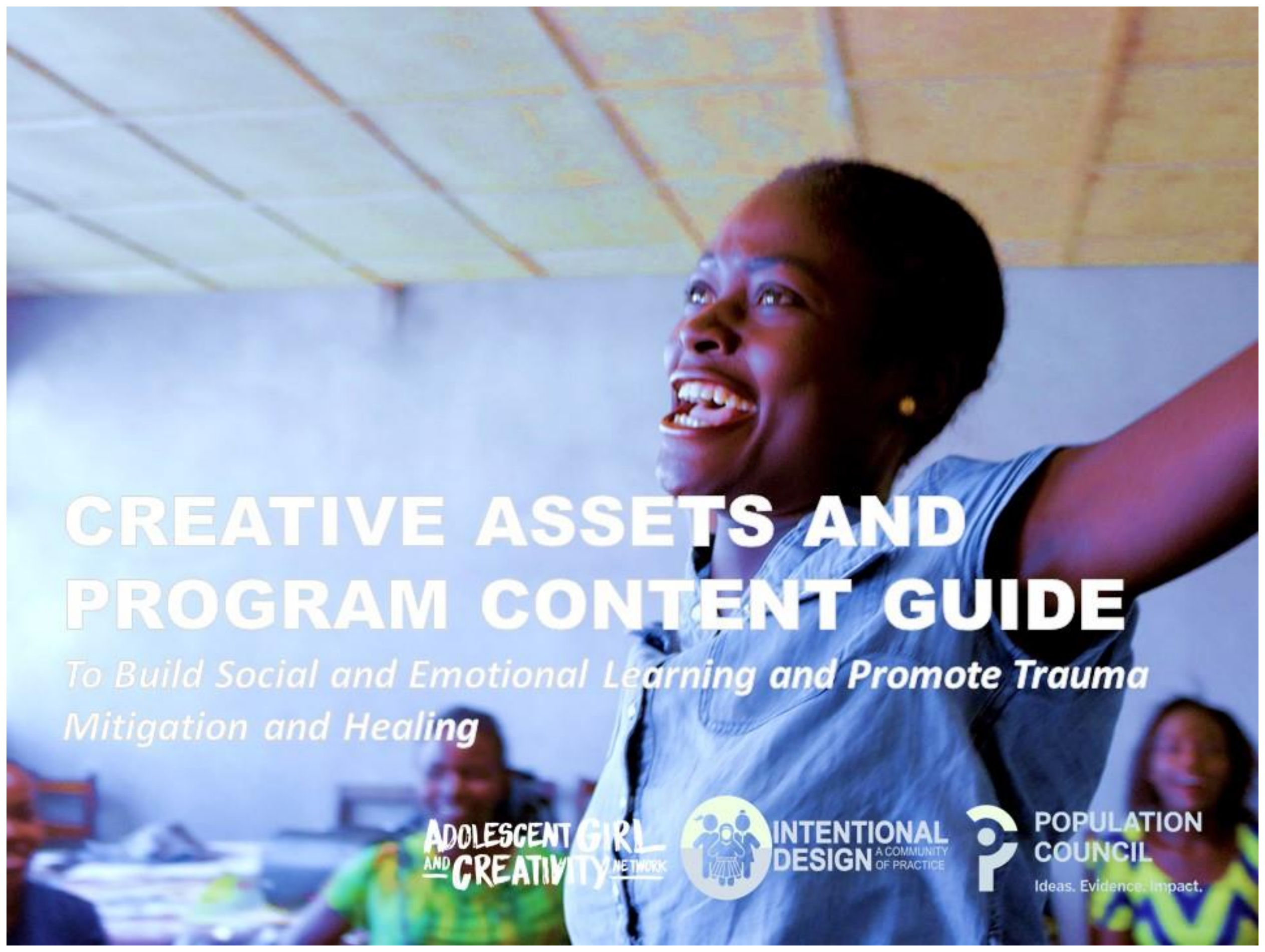


developed by

the Adolescent Girl and Creativity Network

convened and supported by

the Population Council's Community of Practice 


\section{Population Council}

1 Dag Hammarskjold Plaza, $3^{\text {rd }}$ Floor

New York, NY 10017

Tel: +1 2123390500

Email: communityofpractice@popcouncil.org

Suggested citation: Adolescent Girl and Creativity Network. 2020. Creative Assets and Program Content Guide: To Build Social and Emotional Learning and Promote Trauma Mitigation and Healing. Eds. Christina Mallie and Sophie Soares. New York: Population Council.

(c) 2020 The Population Council, Inc.
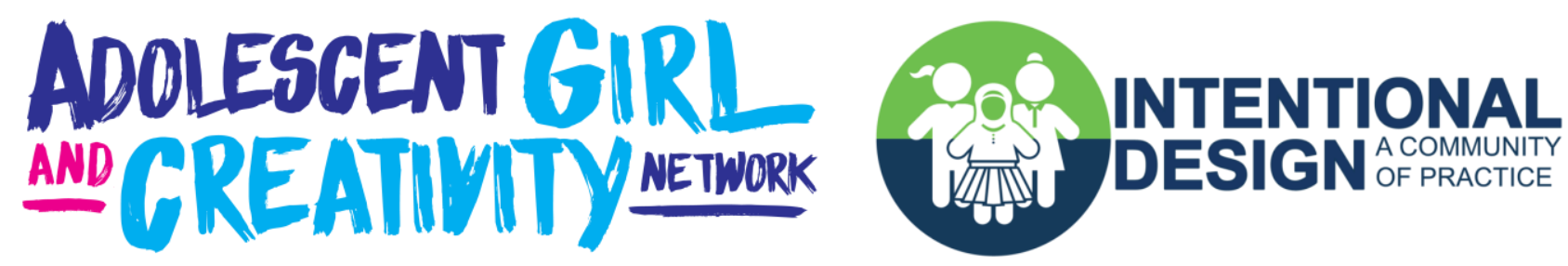

Cover Photo Description: Participant Aziza engaging in s large sounds and gestures for the activity "Raising Your Voice and Expanding Your Body with Musical Chairs" during Colors of Connection's Tunaweza Portraits Project, Goma, DRC 2019. Photo Credit: Bernadette Vivuya 


\section{About the Adolescent Girl and Creativity Network}

The Adolescent Girl and Creativity Network (AGCN) is a working group composed of 13 organizations and 21 individuals, convened by the Population Council's Community of Practice. Led by Christina Mallie of Colors of Connection, the art-driven group is comprised of researchers, educators, policy analysts,

humanitarian aid and development workers, and, in majority, artists. The artists work in a range of mediums - including dance, painting, sculpture, yoga, circus, photography, and more - and a high proportion apply those art forms in humanitarian settings to strengthen communities and mitigate risk. Convened since 2016 , the members have combined their experience in a broad range of countries, contexts and languages to collaboratively generate ideas and innovate program content and activities around how the arts and creativity could affect change among the poorest girls in the poorest communities.

Member Organizations:

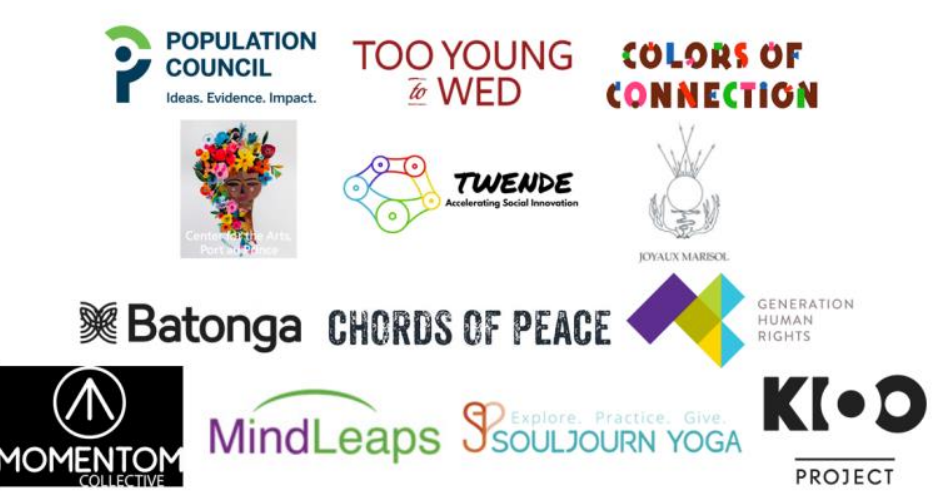

Individual Members:

Christina Mallie, Chair to the Adolescent Girl and Creativity Network (AGCN), and Founder and Director, Colors of Connection

Jordan Ashley, Founder and Executive Director, SoulJourn Yoga Foundation

Sarah Benavent, Associate Director, Orbis International

Nicole Fabienne Bohl, Independent Child Protection Consultant

Gabrielle Bonneville, Co-Founder, Momentom Collective

Judith Bruce, Director of the Community of Practice and Senior Associate, Population Council
Anna Clark, Pilates Instructor \& Founder, WaNgui Project Rebecca Davis, Executive Director, MindLeaps

Nadia Fazal, PhD Candidate in Social \& Behavioural Health Sciences at the University of Toronto

Elana Haviv, Executive Director and Founder, Generation Human Rights

Miranda Leigh, Founder and Creative Director, Joyaux Marisol

Stephanie Cate Lord, Term Member, Council on Foreign Relations, and Founding Executive Director, Batonga Foundation Eleanor Milburn, Assistant Director, MindLeaps

Anu Nagaraj, Post-Production Producer \& Ceramicist, Atelier Anu

Babita Patel, Founder \& Executive Director, KIOO Project

Christina Piaia, Emeritus Board Member, Too Young to Wed
Connor Patterson, Founder \& Director, Chords of Peace Stephanie Sinclair, Founding Executive Director, Too Young to Wed

Sophie Soares, Project Manager for Community of Practice and Staff Associate, Population Council

Nadia Todres, Founder and Executive Director, Center for the Arts, Port au Prince

Deborah Tien, Member, Steering Committee of the International Development Innovation Network (IDIN) and (former) Executive Director, Twende

Juliet Young, Consultant, Adolescent Education and Engagement 


\section{About the Population Council's Community of Practice}

The Population Council's Community of Practice was established in 2013, building on 15 years of prior research on the conditions of girls at the highest risk of the worst outcomes. The Community of Practice helps strengthen the capacity of different actors to design, implement, and evaluate effective, scalable programs that build the protective health, economic, cognitive, and social assets of adolescent girls, particularly the most off-track. We work with institutions ranging from large, multilateral and bilateral organizations and governments to small community-based organizations and national or regional networks. We are committed to providing and supporting the thoughtful use of tools that include:

- country-specific adolescent data guides;

- instructions on how to define the universe of girls in a community and assessing who is reached by programs and services;

- toolkits for designing, implementing, and evaluating girls' programs including identifying the best methods of recruitment, assessing community safety and resources to pinpoint safe space venues, and supporting the recruitment, training, and retention of mentors;

- recommendations for program approaches to build girls' assets;

- $\quad$ and examples of adaptable program content for girls' programs on topics from reproductive health to financial literacy.

As of 2020, the Council has worked with over $\mathbf{1 0 0}$ partners in $\mathbf{4 3}$ countries to reach girls in higher concentrations with measurable results, building the foundation for effective, scalable programs for girls. The Council's efforts have helped to shape policies and programs of nongovernmental organizations, international agencies, and governments around the world to improve girls' lives. For more information about the Community of Practice, please visit:

https://buildcommunity4girls.org. 


\section{Table of Contents}

Part I: Creative Assets

A. Background .8

B. Methodology 9

C. Comments and Resources for the Creative Assets...

Part II. Creative Program Content.

A. Creative Asset Cards

B. Creative Program Content Activities 


\section{A. Background}

\section{History}

In 2016, the Adolescent Girl and Creativity Network was commissioned by the Population Council's Community of Practice to utilize their wealth of knowledge and field experience to critically examine, adapt, and expand the Council's Building Assets Toolkit $\subseteq$ and complementary Asset Exercise. The question was: what are the essential assets pertinent to the most at-risk girls and how can they be built through creative techniques/activities? The following guide is the result of that activity - thirteen creative assets and fifty activities - program content - to build social and emotional learning, mitigate and manage trauma, and promote healing.

\section{Why Creativity?}

The field of creativity has enormous potential to positively contribute to programming for adolescent girls. Creativity is accessible - it is found in different forms in all cultures. And it can be made even more accessible, by innovatively using every day and readily available materials to create. In creating this guide and its contents, it was critical to think of creative activities that could be adapted to diverse and lowresource contexts.

Because seeking mental health services can be stigmatizing, integrating creativity can be helpful in reducing stigma. With the integration of creative activities, participants and community members may able to express themselves or articulate their trauma in a non-stigmatizing way, while simultaneously developing artistic and other skills (Malchiodi 2008). Creativity can be an avenue by which participants can also contemplate and reflect on experiences outside themselves - though they might relate and know those experiences personally - without implicating themselves and allowing for open and unstigmatizing dialogue (Lombardi and Duncan 2019).
An asset is a form of knowledge, skill, and/or resource that is either internal or external. As defined by the Population Council, and based on Amartya Sen's capabilities approach, an asset can be understood to be a "store of value that is related to what a person can do or be (their 'human stock')." Specific to adolescent girls, assets can mediate risks for girls and help them to function, survive and thrive in their economic and social environments. Assets can protect adolescent girls from potential threats and open up more opportunities in her life. A strength of the assetbuilding approach is that it is creates a basis for positive, solution-based, and contextually relevant programming for adolescent girls.

The Building Assets Toolkit $@$ has identified 100 assets that fall under the four broad categories of human/health, social, economic, and cognitive. However, many of the assets are cross-cutting and fall under numerous categories. The Building Assets Toolkit includes a building-assets exercise designed to help practitioners to determine, through a participatory process, which assets are most relevant to the adolescent girls and at what age an adolescent girl should have these assets (Bruce et al. 2015).

Creativity, as an activity, is also often group-based making it compatible in contexts in which healing processes, personal problems, and health concerns are already often a group or community experience (Kira et al.2012). We know that there are many learning and therapeutic benefits to group-based activities, particularly among those who are like-situated. Trauma itself can perpetuate a survivor's isolation, anger, depression, guilt, and more, but a group environment and interaction offers those who have endured trauma a safe space relate to others going through a similar experience, increases their access to resources, and, 
possibly, eases emotional stress. Since creative activities frequently facilitate group interaction and collaboration, they are a natural fit in contexts in which segment-specific programming is essential.

Research on the impact of mental health and psychosocial support interventions on people affected by humanitarian emergencies, found that group-based and segment-specific programs were a significant factor in facilitating and implementing mental health and psychosocial support (Feinstein International Center 2017). (Group-based approaches are also a more viable option in resource-poor environments where there are limitations on cost, access, competency, bias and scale issues for mental health.) To this same effect, group-based creative activities have been found to build social connections and relationships (Barndt 2008). Individual creative activities can also help the individual regulate their emotions, calm their mind and body and be able to attune and connect with others (Malchiodi 2007).

While creativity provides an avenue for adolescents to build a relationship outside of themselves, it can also deepen their connection within as well; creativity is linked to personal growth and emotional health in adolescents. Amidst profound and rapid physical/sexual, psychological and cognitive changes of adolescence make it an intense time of transformation and crisis where there is high vulnerability as well as potential. A growing body of research also connects creativity to overall health and healing from trauma (Huss et al. 2012). Engagement in creativity is shown to have number of positive effects on mental, physical, social, emotional, and spiritual health (MacNaughton et al. 2005). Indeed, art itself is argued to be a determinant of health and is recognized as being involved in direct healing via processes such as art, drama, movement, and music therapies (Lander and Graham-Pole 2008).

The creative assets and content meet central criteria then: accessible, non-stigmatizing, group-based, and health-oriented. Though they are not, within themselves, tools for therapy, diagnosis, or measurement of trauma, their use has therapeutic benefits.

\section{A Trauma-Informed Approach}

We are cognizant that in the contexts in which this toolkit is designed to be used, girls will likely have experienced trauma, making it crucial for activities to be based in a trauma-informed approach in order to promote healing and prevent further harm. Trauma-informed care focuses on three basic principles to help the individual in their growth and healing from trauma: establishing a sense of safety, fostering positive connection to others, and teaching the management of emotions. These three elements are common to most approaches to treating trauma in children and are considered crucial for healing to be able to take place. Relevant to work of the AGCN, these principles can be addressed and nurtured in non-clinical settings (Bath 2008). The tools developed by AGCN members prioritize these three principles: safety, fostering positive connection, and teaching the management of emotions.

\section{B. Methodology}

\section{Establishing Criteria}

The Population Council's Asset Framework provides the basic criteria an asset must meet (Bruce et al. 2015). See Table 1. 


\section{Table 1. Population Council's Asset Framework}

\section{A form of knowledge, skill or resource}

Developed internally or built externally

Transferable to other areas of a girl's life, helping to protect her from risks and/or create opportunities for her.

Adaptable to diverse cultural contexts had similar aims to help the nine participants recall to their personal memories of the positive impact creativity had during their adolescence, distilling those memories into several essential creative assets. The work of the nine members resulted in eight potential creative assets. engaged nine members of the AGCN in a workshop with the objective of identifying
With this as our basis, the AGCN drew on field experiences and practical knowledge to expand on the criteria to which a creative asset must adhere. Table 2 details those additions.

\section{Workshop: The Perfect Creative Moment}

After the criteria were defined, Christina Mallie essential creative assets. Called "The Perfect Creative Moment," this workshop was adapted from one called "A Perfect Summer Day" by the photographer and human rights educator Peter Lucas. Lucas' workshop was designed to help people recall their positive personal memories of childhood and to connect these, as relevant,

These creative assets were then reviewed and edited with input from the network members. Five more creative assets were added by AGCN members at this stage as well.

The final list of assets is found in Table 3.
Table 2. AGCN's Additional Requirements for

Adaptable to illiterate populations

Accessible to populations who may have no prior experience in the arts

Accessible in low-resource environments in

Focused on promoting self-expression vs. developing a technical skill

Focused on building confidence

Have a potential therapeutic impact terms of materials and technique 


\section{Table 3. Thirteen Creative Assets}

1. Know how to recognize/identify emotions in herself and others

2. Know how to express feelings with and without words

3. Know how to self-soothe

4. Know how to gain understanding of herself, her relationships, and her experiences.

5. Know that making mistakes is an opportunity to learn

6. Be able to imagine and express herself in a different role than what is socially assigned

7. Know how to tell a story

\section{Comments and Resources for the Creative Assets}

Useful comments and resources for each asset have been gathered below. We recommend that you review this information as you are using the creative asset cards to ensure you understand the evidence that reinforce each asset's potential value. A final list of resources can be found at the end of this guide.

\section{Creative Asset 1. Know how to identify emotions in herself and in others}

Adolescence, for both boys and girls, is an intense time of both transformation and crisis, a time that psychologists recognize as being one of rapid and profound physical/sexual, psychological and cognitive changes. This contributes to an experience of complex and intense emotions (Emunah 1990). In particular, for the adolescent girl, she may experience emotional challenges in navigating social interactions. Starting in early adolescence, she is likely to be socialized into the dominant gender norms of her community. For example, one study found that adolescent girls reported much denser (generally negative) transitions during this period including school-leaving, sexual initiation and coercion, and child marriage (Mensch et al. 2011; 2013). These challenging and adverse circumstances indicate that the adolescent girls' emotional well-being can be deeply at risk. This is also a period that is characterized by increased social isolation for adolescent girls and without proper emotional support systems and social interaction in their lives, their risks are heightened (McCarthy, et al. 2016).

But, research in the field of art therapy has found that creative expression can be a helpful tool to connect adolescents to their emotions (Malchiodi 2007). This can include the ability to perceive emotions and understand and regulate them (components of emotional intelligence). These assets, research has found, are 
correlated with overall emotional well-being, which itself can promote a more resilient and healthy response to some of the life challenges mentioned above (Schutte, et al. 2002).

\section{Creative Asset 2. Know how to express feelings with and without words}

Research in the field of art therapy suggests that creative expression can connect us to our emotions in ways that may not be accessible through language, helping people to access emotions and sensations that were previously unknown and/or reside in the unconscious (Malchiodi 2007). Creativity not only can connect us to our emotions, but it can support us and be a mechanism to channel emotions in a productive way. Drama therapist Renée Emanuh, Ph.D., asserts that, "creative arts activities can help adolescents achieve a sense of mastery over their emotions" (Johnson 1990). Research has also found that making art can have a transformative effect emotionally, that it can feel cathartic and relieving, meditative, or mood enhancing (Malchiodi 2007).

\section{Creative Asset 3. Know how to self-soothe}

Trauma and post-traumatic stress are frequently a part of living while girl. According to the World Health Organization (WHO), one in every three women globally has experienced physical or sexual abuse, and one in every five women has reported being sexually abused as a child (World Health Organization 2013; 2014). Neuroscience research has found that due to trauma and post-traumatic stress, different functions of the brain are frequently limited. Depending on the severity of the stress, functions of the brain can be compromised including motor coordination, attunement and attachment to others, cognition, and communication (Perry 2006).

To enable greater brain functioning, therapeutic activities that engage the five senses of sight, touch, smell, taste, or sound have been found to be effective. These activities can help to slow rapid breathing or heart-rate and bring one into the here and now, reinforcing a sense of safety (Malchiodi 2012). Stress and trauma are experienced and stored in the body and so activities that engage the body are able to address the experience of stress and have a soothing effect. For example, research in the field of art therapy shows that some creative activities lead to an increase in serotonin in the brain and that other creative activities lead to a slower heart rate and respiration that helps to induce relaxation (Malchiodi 2007).

\section{Creative Asset 4. Know how to gain understanding of herself, her relationships and her experiences}

As mentioned, adolescence for both boys and girls is an intense time of both transformation and crisis, a time that psychologists recognize as being one of rapid and profound physical/sexual, psychological and cognitive changes. This contributes to an experience of complex and intense emotions (Emunah 1990). In addition, for the adolescent girl, she is likely to experience challenges in navigating her social domain, an increasing number of negative life events, and insufficient social support (McCarthy, et al. 2016).

Creative expressions are unique to the person who has created them and they can lead to an expanded sense of a girl's self, and the people, places and things around her. Art therapist Cathy Malchiodi reflects: "Art inevitably tells our personal stories in all their dimensions: our feelings, thoughts, experiences, values and beliefs" (Malchiodi 2007). Research in the field of art therapy suggests that visual creations like drawings and paintings can have the particular effect of showing the creator something about herself or her situation and confronting her with what she has created. According to art therapist Bonnie Hirschhorn, for an adolescent girl, the creative process has the potential to be a path to empowerment. When she is able to gain awareness about herself and her environment, this can have the effect of encouraging her to make change for herself (Hirschhorn, personal communication 2016). 


\section{Creative Asset 5: Know that making mistakes is an opportunity to learn}

Cultivating the skills and values of risk-taking, experimentation, uniqueness and perseverance can be helpful for adolescent girls to advance in their development. Research in the field of social psychology has found that girls and women are negatively impacted by the stereotype that they are inferior to boys and men, such as less intelligent, strong, or capable. This stereotype threatens to lead to women and girls' underperformance academically, socially, etc. because they are more likely to perceive mistakes and failures as evidence of an innate deficiency instead of part of a learning process or a step to success (Steele 1997). Girls need to learn to take risks, accept making mistakes, and know that her performance doesn't reflect her self-worth or ability to succeed, allowing her to move beyond restrictive gender norms and discriminatory beliefs about her abilities.

Research suggests that engaging in creativity could be beneficial for women and girls to persevere in their endeavors despite the societal beliefs of their inferiority. Malchiodi has found that creativity encourages risk-taking and experimentation. Many art forms are based on the goals of personal expression thus drawing positive attention to the self beyond societal definitions. In the arts, uniqueness and perseverance are valued above conforming to a particular standard, which can encourage girls to make an effort and take risks despite societal beliefs (Malchiodi 2007).

\section{Creative Asset 6: Be able to imagine and express oneself in a different role than what is socially assigned}

Rigid gender roles are assigned to both girls and boys that restrict their roles and behaviors. For adolescent girls, gender norms proscribe girls' mobility and their social interactions with boys and men, particularly beginning in early adolescence (McCarthy, et al. 2016). Being able to imagine herself in an alternative role, or to enact a different role than what is socially expected of her is an important step toward her empowerment. In particular, adolescence is accompanied by significant cognitive growth in the individual in his/her ability to think beyond the present and to imagine the future (Emunah 1990). As art therapist Cathy Malchiodi points out, imaginative thinking naturally happens in the creative process, as it enables one to find innovative solutions and transformation. Creativity assists "individuals in moving beyond their preconceived beliefs through experimentation with new ways of experimentation and experiences" (Malchiodi 2005). Malchiodi adds that, "pushing limits, inventing, breaking down boundaries, and rejecting accepted assumptions" can be one of the most central highlights of being creative (Malchiodi 2007). The imagination, which is activated by creativity, has been found to help create new self-perspectives and beliefs (Greene 1995).

\section{Creative Asset 7: Know how to tell a story}

Telling stories is an ancient and traditional process that exists in many cultural contexts. Narrative gives meaning and structure to one's experiences. Particularly for trauma survivors, telling one's story can help in the healing process (Denborough 2014). According to social psychologists Pennebaker and Seagal, "forming a story about one's experiences in life is associated with improved physical and mental health across a variety of populations" (Pennebaker and Seagal 1999). Research has shown that in some settings girls' entry into adolescence is accompanied by a loss of voice, a struggle to take her contributions to conversations seriously, and difficulty to listen and comprehend to her own thoughts and emotions. This research suggests that contexts need to be created that nurture girls' voices (Denner, et al. 2005). Sociologist Tassadit Yacine, in her studies of creativity and women, points out that poetry and song can be a pathway for women to affirm their individual identity and to reverse the societal patterns of submission to men, silence, and complicity in their domination by the patriarchal society (Yacine 2001). In Colors of Connection's project Courage in Congo in Goma Democratic Republic of the Congo (DRC) participants were encouraged to tell their 
stories through drama, drawing and painting. In the words of one participant, "I want to draw to tell my story. I want people to ask me about my drawing and then I can them about it and I can express my story" (Colors of Connection 2016). Projects such as this one can support and foster girls' voices.

\section{Creative Asset 8: Know that she has things in common with others in her community}

Creative engagement can help to highlight similarities, rather than differences, one might share with others. When people create art together or share their art with others, they may find themselves expressing a shared experience and discover that they have things in common. This can have therapeutic and healing outcomes (Malchiodi 2007). Adolescents commonly experience alienation - a sense of powerlessness, meaninglessness, negative self-image and cultural estrangement (Johnson 1990). Adolescent girls in particular are more socially isolated than boys and lack opportunities to connect with their peers (Austrian and Ghati 2010). In the example of Colors of Connection's project Courage in Congo in Goma Democratic Republic of the Congo (DRC), creative activities fostered strong connections between adolescent girl participants. Participants connected with each other through collaborative arts activities and individual activities that were shared with the group. One community stakeholder observed that "this kind of project reinforces cohesion, and we definitely observed this among the girls. From what we saw, there were almost no barriers that remained between them by the end" (Colors of Connection 2016). Creating, sharing and receiving art, being exposed to a shared experience can lead to instilling hope, building social support, creating a sense of universality of common worries, problems and fears, creating catharsis and fostering altruism (Malchiodi 2007).

\section{Creative Asset 9: Know how to establish a safe space for her to be creative}

Personal and safe spaces are needed to be creative. Literary feminist Virginia Woolf's famously wrote that "a woman must have money and a room of her own if she is to write fiction" (Woolf 1929). Similarly, adolescent girls need to cultivate safe personal spaces to be creative in a societal environment that routinely denies them the time and space to do so. On the other hand, acting as a two-way street, some creative activities have been found to help adolescent girls understand and establish safe and personal space. One pilot project on GBV prevention and response in Ecuador with Colombian refugee boys and girls worked with dance, meditation, journal writing and drawing and focused on the core concept of establishing personal space. Female participants reported developing a more positive relationship with themselves, their bodies, and an understanding of the right to decide what happens in her space including to her body (Women's Refugee Commission 2017). In a broader perspective, sociologist Tassadit Yacine has called attention to the capacity of creativity to engender new thoughts, feelings, and ideas into the world, that are authored and owned by the female creator, reversing the established order (Yacine 2001).

\section{Creative Asset 10: Know how to establish a safe space for a group}

Fostering safe space for girls is important during adolescence. As girls enter puberty their movement in public spheres becomes more and more restricted and controlled as perceived and real threats to her body including sexual assault and rape increases. Girls have identified smaller and smaller areas that they consider only somewhat safe spaces in their communities each subsequent year of puberty. Because girls lack adequate safe spaces, and are restricted in their access to public spheres, they are more socially isolated, and have limited opportunities to meet peers, and to engage in activities to further their human development (Hallman et al. 2015). Research shows that girls who have stronger social networks have less likelihood of sexual harassment and violence, highlighting the need for girls to develop stronger social networks (Bruce and Hallman 2008). 
Creativity can be used as a tool for girls to develop a safe group space among themselves. Jean B. Peterson LCSW, ATR-BC, T.E.P., suggests group warm-up exercises to increase spontaneity and to help bring people into the here and now and be more authentic in expressing their feelings. According to the founder of sociometry ${ }^{1}$ and psychodrama, ${ }^{2}$ Jacob L. Moreno M.D., spontaneity is the opposite of anxiety. When spontaneity increases, emotions of anxiety decreases (Peterson 1982). Creativity can provide an alternative and positive way to language for individuals in a group to communicate and connect. For example, studies have found that adolescents who experience shyness, anxiety, depression and anger can develop positive relationships with their peers more easily though a structured program with artistic activities (Guetzkow 2002).

\section{Creative Asset 11: Know how to occupy, hold and defend space physically socially and psychologically}

Gender norms prescribe that women and girls act in submissive ways in their body language and conversational style. Having lower social status than men and boys, girls are taught to take up less physical, conversational and psychological space and to have permeable boundaries that can be subsumed by men. A sense of invisibility is even encouraged (Kaschak 1992). Studies found that when girls enter adolescence, they become quieter, less expressive, take up less space in public, and become more focused on social acceptance (Pecora and Mazzarrella 2001). Helping girls learn how to occupy physical, social, and psychological space and to develop boundaries is important. Studies have found that activities related to these goals can help adolescent girls to build protective factors in their lives including being more aware of one's environment, being able to better assert oneself, and responding to gut intuitive reactions in uncomfortable situations (Peace Over Violence 2013). Creative activities such as mindfulness, the dramatic arts, dance, and ones that engage the emotions can be used to cultivate these protective factors.

\section{Creative Asset 12: Know that social media and culture often represent women and girls in ways that can be harmful to her health}

Social and other media, specifically fashion and beauty advertising, often represent women and girls as sexualized objects (Stankiewicz and Rosselli 2008). Advertising also includes harmful messages that encourage women and girls to modify their bodies. One of the most common body modifications in the world today that impacts women and girls of color is skin bleaching. Beyond the message of racial inferiority that skin bleaching advertisements perpetuate, one study on women in Tanzania points out that skin bleaching has been found to be correlated in irreversible skin damage among other health risks including infertility (Lewis et al. 2011). There is a need for adolescent girls to be able to reflect on their experiences, thoughts and responses to this onslaught of messages about who they are and should be in society. A space is needed in girls' lives to develop critical awareness of media and social culture.

Creativity has been found to allow individuals to digest and communicate information differently, and to experience their world in new ways (Eisner 2008). Creativity fosters more critical thinking, possibly offering girls' the skills to protect themselves from harmful messages of social media (Mayer 2002). Creative activities have the potential to help girls to reflect on power dynamics, and the representation of women and girls in the media and social culture.

\section{Creative Asset 13: Be able to identify what beauty means to her}

\footnotetext{
${ }^{1}$ A qualitative method developed by Jacob L. Moreno for measuring social relationships.

${ }^{2}$ An experiential approach to therapy that utilizes role playing and guided drama.
} 
Adolescent girls can develop positive alternative relationships to their bodies outside of the media through creativity. Creativity is a pathway for adolescent girls to connect to their inner thoughts, feelings, experiences and perceptions. This can help adolescent girls to develop a different relationship with themselves, one that is based on their individuality and self-worth, encouraging them to give value to aspects of themselves beyond societal norms (Malchiodi 2007).

Imagination plays a key role in this process. As art therapist Cathy Malchiodi suggests, imaginative thinking naturally happens in the creative process, as it enables one to find innovative solutions and transformation. Creativity assists "individuals in moving beyond their preconceived beliefs through experimentation with new ways of experimentation and experiences" (Malchiodi 2005). Malchiodi add that, "pushing limits, inventing, breaking down boundaries, and rejecting accepted assumptions" can be one of the experiences of being creative (Malchiodi 2007). The imagination, which is activated by creativity has been found to help create new self-perspectives and beliefs, as Maxine Greene, points out, an educational philosopher and social activist (Greene 1995). In addition, the arts have been found to encourage a search for beauty externally and internally, that can encourage adolescent girls to find beauty within themselves (Eisner 2002). 


\section{Part II. Creative Program Content}

\section{A. Creative Asset Cards}

Know how to recognize/ identify emotions in herself and others

\section{ADOLESGENT GIRL} ANG GREATIYITYYNELOQR

Know how to self soothe

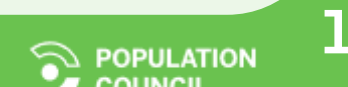

Know how to express feelings with and without words

\section{ADOLESGENT GIRL}

Know how to gain understanding of herself, her relationships and her experiences 
Know that making mistakes is an opportunity to learn

Know how to tell a story

Be able to imagine and express herself in a different role than what is socially assigned

\section{Know that she has things} in common with others in her community 
Know how to establish a safe space for her to be creative
Know how to establish a safe space for a group

Know how to occupy, hold and defend space physically, socially, and psychologically

Know that social media and culture often represent women and girls in ways that can be harmful to her health 
Be able to identify what beauty means to her 


\section{B. Creative Program Content Activities}

The creative program content activities were contributed by members of the AGCN, including Gabrielle Bonneville, Nicole Fabienne Bohl, Elana Haviv, Jordan Katz, Miranda Leigh, Christina Mallie, Eleanor Milburn, Babita Patel, and Connor Patterson. The following AGCN members provided peer review of these activities based on their personal experiences with creativity and on their work with adolescent populations in multiple regions: Gabrielle Bonneville, Nicole Fabienne Bohl, Nadia Fazal, Elana Haviv, Jordan Katz, Miranda Leigh, Christina Mallie, Eleanor Milburn, Babita Patel, Connor Patterson and Sophie Soares.

In this section you will find fifty creative program content activities to build the thirteen creative assets. There are multiple activities that can build each asset. Table 3 below shows you the pairing of asset to activity by activity number; if you know which asset you are learning in building, you can find the specific activities that relate.

\section{Table 3. Program Content Activities Per Creative Asset}

\begin{tabular}{|c|c|}
\hline Creative Asset & Program Content Activity to Build Creative Asset \\
\hline 1. Know how to identify emotions in herself and in others & $1,2,4,24,27$ \\
\hline 2. Know how to express feelings with and without words & $2,38,22,23,24,27,28,30,42,48$ \\
\hline 3. Know how to self-soothe & $8,9,10,11,14,15,22,23,24,44$ \\
\hline $\begin{array}{l}\text { 4. Know how to gain understanding of herself, her relationships and her } \\
\text { experiences }\end{array}$ & $2,3,17,19,22,23,24,50$ \\
\hline 5. Know that making mistakes is an opportunity to learn & $16,42,43$ \\
\hline $\begin{array}{l}\text { 6. Be able to imagine and express oneself in a different role than what is } \\
\text { socially assigned }\end{array}$ & $18,24,26$ \\
\hline 7. Know how to tell a story & $17,21,26,29,30,31,32,33,34,35,36$ \\
\hline 8. Know that she has things in common with others in her community & $1,14,15,17,23,27,28,29,30,31,32,33,34,35,36,40,42,45,48$ \\
\hline
\end{tabular}


11. Know how to occupy, hold and defend space physically socially and psychologically

12. Know that social media and culture often represent women and girls in ways that can be harmful to her health.

\section{General Tips for Engaging with the Activities}

- Do warm-up exercises that highlight the universality of the experiences of members of the group to build solidarity and establish as safe space.

- Allow for spontaneity - the activity might take different directions depending on the group and their interests, but spontaneity can allow for more authentic expression and is directly linked to decrease in anxiety. Group warm-up exercises can also increase spontaneity.

- Consider using different artistic mediums to work with different emotional states. Clay and paint are more spontaneous and are recommended for people struggling with depression, as they are likely to be containing their emotions too much. Working with colors is also recommended for depression as it can help to bring out emotions. Materials that are easy to control like pencils, building materials or sand play as well as repetitive techniques, and soft materials can be soothing for those struggling with anxiety.

- Listening to relaxing music, making sounds or humming can help to reduce stress and regulate the heart rate. Music can be played leading up to or during an artistic activity to help establish a calming and safe environment.

- If working with mixed gender groups, place girls into a guaranteed leadership role and boys into an opportunity to be a student to change typical gender norms.

- Working with materials that are traditionally reserved for men and boys such as masonry, sculpture, and carpentry can help girls to get in touch with different types of expression that are outside of their traditional gender role.

- When presenting and sharing artwork at the end of an activity, encourage girls to identify common themes or similarities within the images or ideas behind the images to show commonalities to build solidarity. Also ask girls to spot differences to acknowledge how each person is different to encourage appreciation of individuality.

- When doing visual activities, particularly creating paintings or murals, encourage girls to create works that are physically large, so that they feel they are expanding into and building out their safe space.

- When doing storytelling activities, allow girls to create fiction or fantasy, as this might be a more accessible way for them to explore their identities and relationships to society and expand beyond proscribed gender roles. 


\section{A Word on Activity Facilitators}

We suggest that facilitators implementing activities be young women, slightly older than the participants and of their community. Their lived experiences and understanding of the participants will help to create effective and contextually appropriate facilitation. Being slightly older than the participants, they can also serve as positive young role models providing perspective into plausible next life steps for participants. Young, relatable women like the girl participants can help to establish a safe space for participants to share experiences, ask questions, or seek help from the facilitators. ${ }^{3}$

\section{Activities List}

The creative program content activities are organized by activity type: language/discussion; breath/awareness; visual; music; drama and storytelling; and movement. Below is the name of each activity sorted by activity type.

With each activity, this guide details the amount of time and type of preparation and materials required (if any), provides detailed instructions, identifies the activity's objective and the source(s) of the activity.

\section{Language/Discussion}

Activity 1: Name the Feeling

Activity 2: I Feel Statements

Activity 3: Big Self - little self

Activity 4: Identify Emotions Through Photographs 30

Activity 5: How You See Yourself vs. How Others See you

Activity 6: Collaboratively Created Group Guidelines

Activity 7: Reading Photos - Multiple Activities

Breath/Awareness

Activity 8: Four-Part Breath

Activity 9: Three-Part Breath

Activity 10: Ocean Breath

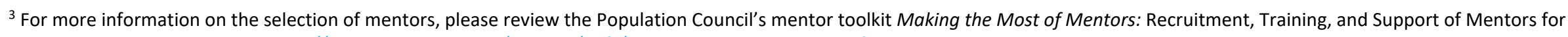
Adolescent Girl Programming: https://www.popcouncil.org/uploads/pdfs/2019PGY MentorToolkit.pdf. 
Activity 12: Canvass Bag Decoration/A Personal Safe Space .......................................... 39

Activity 13: A Collage of Calm and Safety ............................................................... 40

Activity 14: Chase the Scribble …............................................................................. 41

Activity 15: Collaborative Doodle ……………………………………………........... 42

Activity 16: Making Shapes ....................................................................................... 44

Activity 17: Magazine Photo Collage …………….................................................... 45

Activity 18: Traditional and Non-Traditional Gender Roles through Photography....... 47

Activity 19: Being Seen/Self-Portraiture ................................................................. 51

Activity 20: Transformed Photo Self-Portrait ................................................................ 54

Activity 21: Telling Your Story, Being Heard ……............................................................ 56

Activity 22: Mandala Drawing .................................................................................... 58

Activity 23: Group Danmala ................................................................................. 60

Activity 24: Goal Setting Using Body Maps .................................................................. 62

Activity 25: Stronger Together Collaborative Work ...................................................... 66

Activity 26: Exploring Non-Traditional Gender Roles Through Drawing ...................... 67

Music

Activity 27: Musical Emotions ..................................................................................... 68

Activity 28: Controlled Expression of Thoughts and Feelings through Improvisation... 69

Activity 29: Finding Stories in Songs........................................................................... 70

Drama and Storytelling

Activity 30: The Wrapper Game

Activity 31: Story Circle 


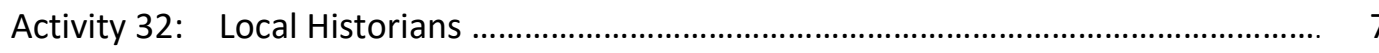

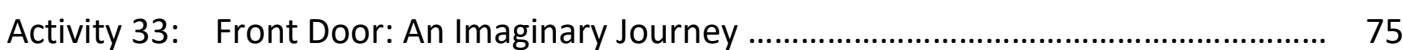

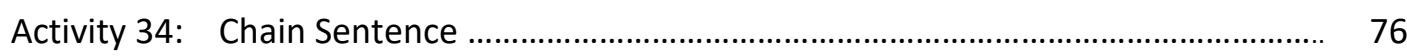

Activity 35: The Autobiography of Anything ........................................................... 77

Activity 36: Proverbs: Wisdom Tales without the Plot ................................................... 78

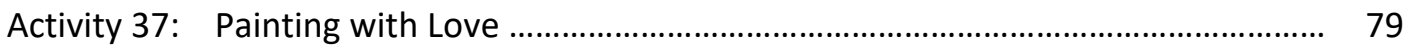

Movement

Activity 38: Tree Pose

Activity 39: High Power Poses ................................................................................. 82

Activity 40: $\quad$ Raising Your Voice and Expanding your Body with Musical Chairs ........... 83

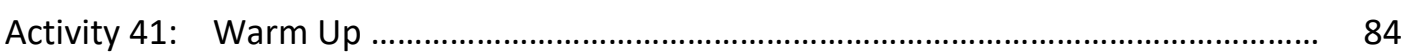

Activity 42: Opposites Improvised Movement .................................................... 85

Activity 43: Balancing Yoga Challenge ........................................................................ 86

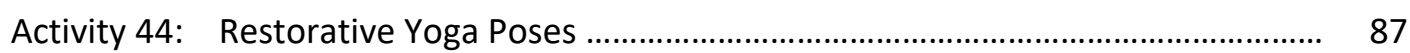

Activity 45: Spectrogram Warm-up .................................................................. 90

Activity 46: Exploring Movement in the Kinosphere ................................................. 92

Activity 47: The Circle Game .................................................................................... 93

Activity 48: The Shape Game ............................................................................. 94

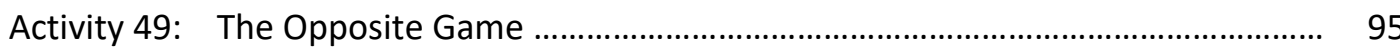

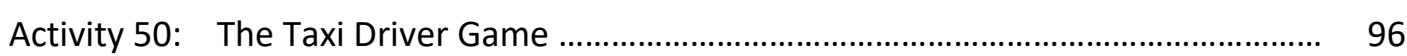




\section{ACTIVITY 1: Name the Feeling}

DURATION: 20 minutes

MATERIALS: Emotions chart

TO PREPARE: Facilitator should think of several examples of emotions to share with the participants in advance

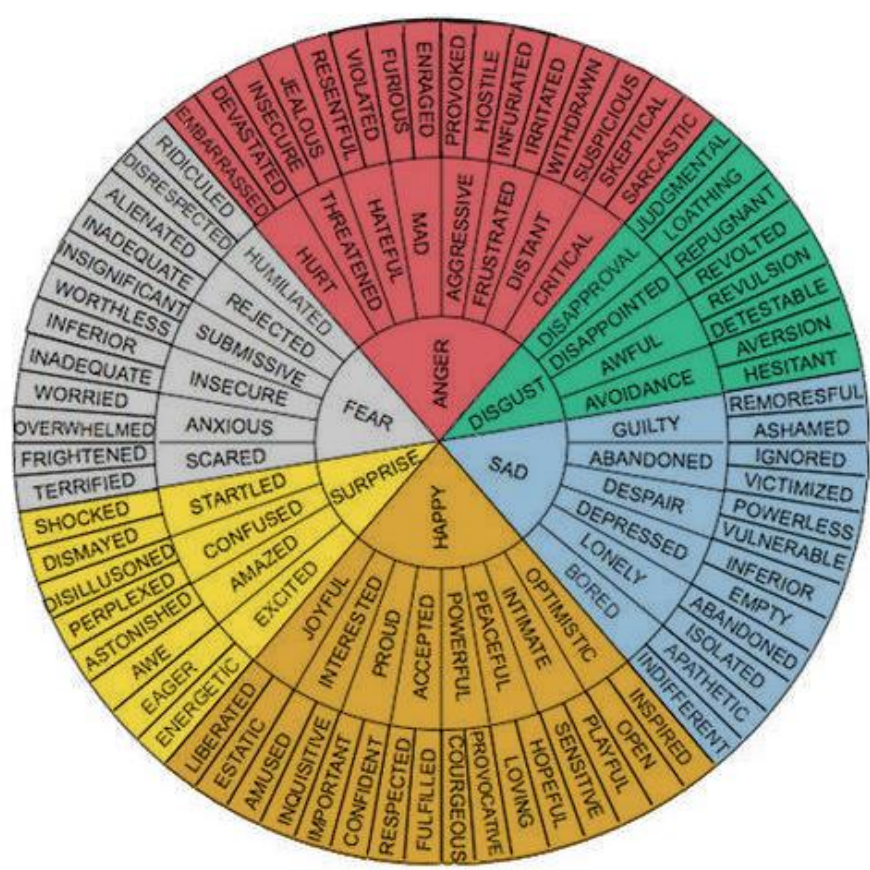

Photo Credit: Ginny Styles 2014 http://westendcounselling.co.uk/emotions/wheel-of-emotion/
Creative Asset 1: Know how to identify emotions in herself and others

Creative Asset 8: Know that she has things in common with others in her community

Source: Christina Mallie, Colors of Connection, www.colorsofconnection.org

Overview: This is a group activity in which participants practice identifying simple to complex emotions.

Objectives: Participants will build emotional intelligence and awareness of their own emotions.

Note: This can be adapted to illiterate populations by speaking the different emotions instead of showing the chart.

\section{Instructions:}

1. Open this activity by emphasizing the tenants of a safe space and the importance of respect for what is shared by participants through this activity.

2. Ask participants to think of a real past experience in which they felt either: happy, surprised, fearful, angry, disgusted, or sad (inner circle of emotions on the chart).

3. To help participants tap into their feelings, following from the heart instead of the head you can lead them through a visualization to get in contact and choose an experience.

4. Ask them to close their eyes or look gently on the ground. For this step, you might demonstrate this first using your own example. 
6. Use any of these variations of direction to guide the participants:

- "Gently drift through your memories. Happy moments, sad moments, confused moments."

- "There is no right or wrong. Focus on the one that comes up for you."

- "Where are you? Are you alone or with others? Are there colors, tastes, smells?"

- "Where is this memory in your body? Where do you carry it? How does it feel? Does it have a shape/color/feeling?"

- "Travel through your body from head to toe and locate the memory."

7. Once they have had some time to reflect quietly, ask each participant to gently open their eyes.

8. As the participants to form pairs and share their experience with their partner. If they do not want to share specifics about their experience, they can choose just to share the name of the emotion.

9. Ask the participants to share their experience and/or the name of the emotion with the larger group. Note: By sharing, they can learn from each other and benefit from each other's experience.

10. Ask each participant to choose one of the more detailed complex emotions on the second circle (see chart) that is more precise in describing the initial emotion they chose.

11. Repeat this to the next outer more complex and detailed circle.

12. Once they have chosen something from the outer circle, encourage them to think if there is a better name for the emotion they shared. It is possible they would label it differently. They may even want to make up a name from scratch! You may want to remind them that this chart, while helpful, is limited and that they know themselves better than anyone.

13. To emphasize the commonalities that participants share with each other, ask the participants to comment on whether any of the experiences resonated with them, and if they have similar experiences that they want to share with the group.

14. Close the activity by thanking everyone for sharing and asking if anyone learned any new names for their emotions. 


\section{ACTIVITY 2: I Feel Statements}

DURATION: Approximately 20 minutes

TO PREPARE: Facilitator should think of several examples to share with the participants.

Overview: This is a group activity in which participants practice identifying simple to complex emotions.

Objectives: Participants will develop awareness of their relationships to others around them.
Creative Asset 1: Know how to identify emotions in herself and in others Creative Asset 2: Know how to express feelings with and without words

Creative Asset 4: Know how to gain understanding of herself, her relationships, and her experiences.

Source: Jordan Ashley, SoulJourn Yoga Foundation, http://www.souljournyoga.com/

Note: Can be done as an individual or group activity.

\section{Instructions:}

1. Begin by sharing examples with the participants. For example: I feel (sad); When you (don't let me share my feelings/tell my side of the story); Because (it makes me feel undervalued); And I would like it if you (listened to me). Emphasize to the participants that there is a form of compassionate communication (also known as non-violent communication), which means that they can practice feeling empathetic towards the person they are confronting.

2. Ask participants to practice voicing these "I feel" statements to each other by partnering up (not in terms of their actual feelings toward each other, just in relation to the original experience/person they were thinking of when they generated the statement).

3. Instruct participants to think of a specific experience with a specific person and to practice filling in the following banks: I feel When you ---- Because - And I'd like it if you

4. After each participant has had the chance to try 2-3 of these with their partner ask the group to come back together. Ask each partner to share with the group their favorite example that their partner shared with them and why it is their favorite.

5. After participants have shared with the group, you can lead a discussion that encourages reflection on the activity by asking questions like: Were you surprised by any of the examples? Is there one you think is useful to you in your life? Which emotion was the least discussed and which was the most and why do you think that might be? 


\section{ACTIVITY 3: Big Self - little self}

DURATION: Minimum 15 minutes

MATERIALS: Colored pencils/crayons and paper (optional)

TO PREPARE: Facilitator should think of several examples to share with the participants.
Creative Asset 4: Know how to gain understanding of herself, her relationships, and her experiences.

Source: Jordan Ashley, SoulJourn Yoga Foundation

http://www.souljournyoga.com

Note: Can be done individually or in a group.

Objectives: Participants will develop perspective of their temporary and big-picture life experiences including emotions and thoughts.

\section{Instructions:}

1. Introduce participants to the Bhakti concepts of little self and Big Self. Provide examples of your little self and Big Self based on the concepts. The concepts are:

- little self: all of what makes us us - our backgrounds, personal history, the things that compose our personalities; but they are also the details that are the most changeable and malleable.

- Big Self: your essence, life force, spirit, soul; the thing that represents the best version of yourself. This is a constant and does not change.

2. Remind the participants that by distinguishing the two, they can see that while their problems, worries, or concerns may seem to be dominating their quality of life, it is not forever.

3. Ask participants to pair up and share with each other the following: How old they are, where they live, something they like, something they feel, and something they are worried about. Then ask them to imagine in 5 years from now, what things might be the same or different about these things?

Note: An adaptation is to have the participants draw what they have described, creating one drawing that focuses on the little self and one drawing that focuses on something that they believe won't change about themselves, their Big Self. 


\section{ACTIVITY 4: Identify Emotions Through Photographs}

\section{DURATION: 20 minutes}

MATERIALS: The emotion chart

TO PREPARE: Facilitator should gather a wide range of images depicting different scenes/people/colors/places/things that express a particular mood.

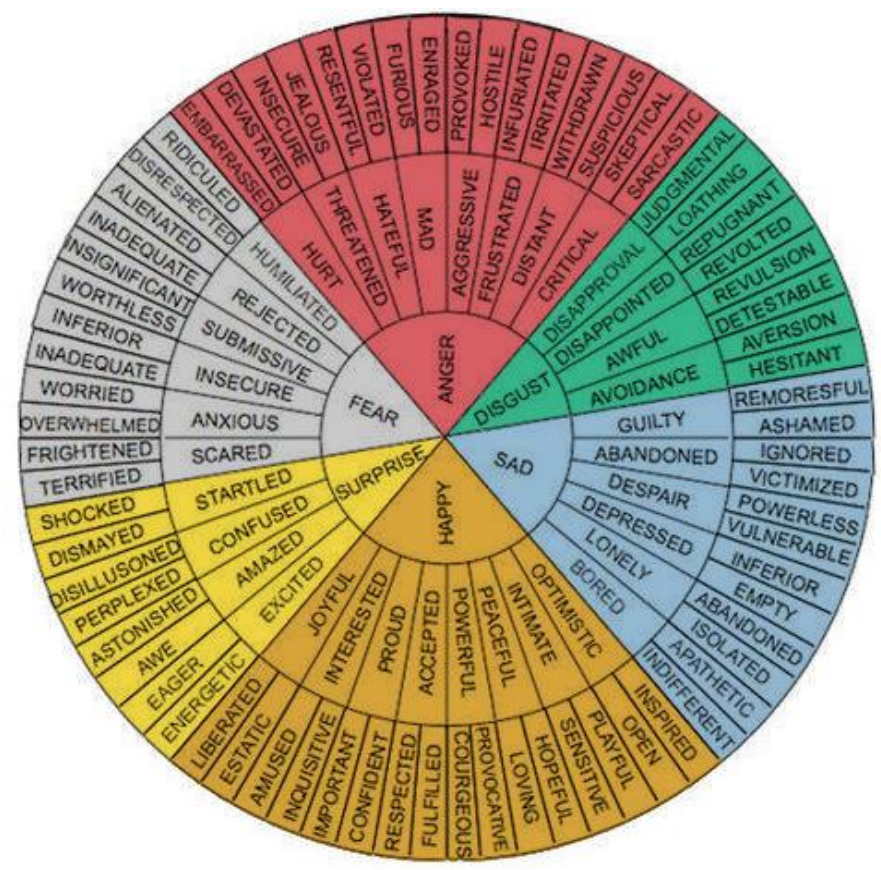

Photo Credit: Ginny Styles 2014 http://westendcounselling.co.uk/emotions/wheel-of-emotion/
Creative Asset 1: Know how to identify emotions in herself and others

Source: Babita Patel, KIOO Project, https://kiooproject.org/

Overview: Identifying emotions through photographs.

Objectives: Participants will understand how to read emotions. Participants will expand their vocabulary for different emotional states.

\section{Instructions:}

1. Call out an emotion, (e.g. joyful, proud, stressed, lonely, etc.) choosing more or less complex ones depending on the capacity of the participants to understand the words. (See the attached emotion chart for options of words.)

2. Have the participants pick out an image that relates to that emotion for them.

3. Have them present the image and the reasoning why they picked that image for that particular emotion.

4. When you or other participants critique others' art work, use this exercise to practice dialog like "I (dis)agree with your answer because..." rather than "I (dis)agree with you because..." to distinguish how one feels about another is not a personal attack. 


\section{ACTIVITY 5: How You See Yourself vs. How Others See You}

DURATION: 60 minutes

TO PREPARE: If possible watch the following link to gain some familiarity with the concept that this activity is adapted from:

https://www.youtube.com/watch?v=XpaOjMXyJGk

Overview: Comparing descriptions of herself with descriptions of how others see her opens up a discussion on self-confidence and different definitions of beauty.
Creative Asset 13: Be able to identify what beauty means to her

Source: Christina Mallie, Colors of Connection, www.colorsofconnection.org

Adapted from the "Dove Real Beauty Sketches with Gil Zamora."

https://www.youtube.com/watch?v=XpaOjMXyJGk

Objectives: Participants will develop awareness that they are often the most critical of themselves. Participants will cultivate awareness of their own beauty.

Note: As this activity can bring up sensitive issues of what a girl might like or not like about herself or others, it should only be done when a safe space has been established in the group (see Activity 6 - Collaboratively Created Group Guidelines for guidelines on creating a safe space).

\section{Instructions:}

1. Introduce the activity by sharing with participants that often we are our own worst critics of not only what we do but how we look. Invite discussion on this, asking if anyone agree or disagrees in the group with this idea.

2. Ask each participant to describe her face as if to someone who is blind. This should be done privately and noted by the facilitator or if the participant is literate can be done by herself by privately writing down her own description of herself.

3. Ask the group to form pairs and to observe each other's faces.

4. Each pair will describe the other person's face to the group as if the group is blind and the facilitator will compare these descriptions to how the participant described herself.

5. Discuss what was different between how people describe themselves and how others describe them. You can pose questions such as: "Did you notice a difference between how you described yourself versus how your partner described you? What were the differences? Was there something someone observed that you didn't notice or know?"

6. You can show the following YouTube link provided above if you have access to internet at the end of the session and if it is contextually appropriate, for example if the participants speak English. 


\section{ACTIVITY 6: Collaboratively Created Group Guidelines}

\section{DURATION: 30 minutes}

MATERIALS: Poster board or large sheets of paper, colored markers

TO PREPARE: Think of potential guidelines and rules that you can suggest if they aren't raised by the participants themselves.

Objectives: Participants will create a sense of safety and community within the group.
Creative Asset 13: Know how to establish a safe space for a group

Source: Christina Mallie, Colors of Connection, www.colorsofconnection.org and Babita Patel, KIOO Project, https://kiooproject.org/

Overview: Collaboratively create group guidelines and make a poster to display them.

Note: This is a good activity to do before other group activities to help establish a safe space, for example at the beginning of a workshop. Building a community within the group can also encourage them to reach out to each other beyond the official workshop or session.

\section{Instructions:}

1. Explain to the participants that they will be creating a set of guidelines or rules that they will agree to follow within this group context with each other and the facilitator. A safe space doesn't have to be a physical space but rather comfort in being with someone who shares the same values.

2. Ask for ideas and write them down as people suggest them, in a way that the whole group can see what you're writing. For example, on a chalkboard or big piece of paper. You can help participants by asking them to think about how they would (and would not) like to be treated by others in the group and about what things might be important for them (and others in the group) in order to feel safe and to feel comfortable learning in this environment.

3. Some potential guidelines that you can suggest as a facilitator, if they are not suggested by others are: one person talking at a time, respecting the opinions of others, not gossiping or blabbing what someone shares, not stealing, etc.

4. Once all of the guidelines have been proposed and have been agreed upon, you and/or the participants can write them on a poster board that is put up or visible during the sessions. If there are low literacy levels in the group, the poster idea may or may not be useful or popular, but even its physical presence can serve as a reminder to your group. You can also adapt the poster to work with low literacy level group by using symbols or pictures to represent the different guidelines. 


\section{ACTIVITY 7: Reading Photos - Multiple Activities}

\section{DURATION: Varies}

MATERIALS: Photos (varies)

TO PREPARE: Varies

Overview: A collection of activities that help to develop photo literacy and critical thinking.
Creative Asset 12: Know that social media and culture often represent women and girls in ways that can be harmful to her health

Source: Peter Lucas https://www.peterlucas.net/

Objective: Participants will develop their visual literacy and critical thinking. There might be other objectives depending on how you set the activity up.

\section{Instructions:}

The following activities are suggested workshops when working with photographs. We hope that both the educator and the participants will approach these activities with an open attitude of receptivity and wonder. To be sure, we want to encourage critical media literacy here and initiate a discussion on the imagery, but we also want participants to appreciate that a photograph is not only something taken, but also something received. The process of receiving an image invites curiosity, contemplation, revelation, and a genuine openness towards other ways of knowing, including valuing ambiguity and uncertainty.

As with all of our educational activities, participants should be encouraged to respond to images in a brainstorming mode where there are no wrong answers, only ideas and questions to be explored.

- Reflecting and Photo Analysis:

Objective: To develop interpretation skills

Instructions: Ask participants to list people, objects, and activities in the photo. Then ask them to describe specifically what is the mood/feeling, what is the story, and what are the relationships in the photo.

- Storyboarding:

Objective: To develop an understand the situation in the photograph and the larger story.

Instructions: Participants agree on a story of the photograph and imagine and draw what came before this particular moment and what came after this particular moment.

- Beyond the Edge:

Objective: To develop an understanding of the context beyond the limits of the photograph. To understand that each photo is only a partial representation of a larger situation.

Instructions: Print photographs with a generous white border on all four sides. Using the four borders of the photo, each participant shares her thoughts 
about what she thinks is happening just beyond the frame of the picture. participants can share their ideas about the larger physical context of the scene in the picture, the presence of the photographer, and any ideas about the before and the after life of the photo.

- Quartering an Image:

Objective: To help participants hone their skills at looking at photographs from one edge to the other and seeing more critically into the photo.

Instructions: Divide an image into four quarters. Break participants into four groups, giving each group a quarter of the image. Each group critically analyzes their quarter and imagines what the entire photo represents. After the groups share their findings with the larger group, they are shown the photograph in its entirety. The class should then discuss any differences or insights they had on the partial and whole image. The class should also talk about how this activity might have helped them to see a little deeper and more critically into a photograph.

- Photo as a Deep Drawer:

Objective: To identify larger issues and perspectives that are expressed in photos.

Instructions: Remind the participants that a photograph is like a deep drawer; there are things on the top that we can easily see and other things that are hidden below. Those things on the surface can inform us, but the deeper issues can emotionally move us - sometimes in ways we cannot explain. Encourage participants to look at a photograph in layers, first ask them to identify the superficial surface elements and then ask them to guess what the deeper perspective and underlying issues might be expressed in the photo. 


\section{ACTIVITY 8: Four-Part Breath}

DURATION: 1 minute or longer as needed

Objectives: Participants will learn to calm the parasympathetic nervous system, soothing the body and the mind and helping to bring them into the present moment.
Creative Asset 3. Know how to self-sooth

Creative Asset 9: Know how to establish a safe space for her to be creative

Source: Jordan Ashley, SoulJourn Yoga Foundation

http://www.souljournyoga.com/Based on Hatha Yoga and Kundalini Yoga

\section{Instructions:}

Lead participants through the following exercise. You can share a version of the following instructions with them and making modifications as needed: Introduce the activity:

"This is called the four-part breath because there are parts: inhalation, internal retention or holding, exhalation, and external retention or holding. There are four equal spaces for inhale, exhale and the space in between to act as transitions. You can think of this as a metaphor for receiving/giving and holding space for the transitions in the interim."

1. Sit with a straight spine in a chair or in a comfortable seated position on the floor. Ideally you want the hips to be a little higher than the knees so that you can find some length throughout the entire spine especially through your lower back. You don't want to be hunched over and want to be open through the chest.

2. To start rest your palms on your knees facing up and draw your shoulders back and close your eyes if that feels comfortable to you. If you feel more comfortable with your eyes open that is fine too.

3. Feel the sit bones connecting down to the earth and feel the top of the head reaching up to the sky.

4. Begin by breathing in four parts in the following pattern:

Inhale 2,3,4

Hold 2,3,4

Exhale 2,3,4 and

Pause $2,3,4$

Repeat the cycle for as long as you wish.

Note: If the pauses are uncomfortable you can adapt this activity to focus on equal parts of inhaling and exhaling for counts of four. 


\section{ACTIVITY 9: Three-Part Breath}

DURATION: 5 minutes modifying to shorter or longer periods as desired.

Objective: Participants will learn to calm the parasympathetic nervous system, decreasing their stress and anxiety. It also helps to bring them into the present moment and into a state of awareness/alertness.

Note: Can be done individually or in a group.

\section{Creative Asset 3. Know how to self-sooth}

Creative Asset 9: Know how to establish a safe space for her to be creative

Source: Sophia Mallie, http://www.yogawithsophia.com/ based on Hatha Yoga

\section{Instructions:}

Lead participants through the following exercise. Start by introducing the activity. Then share a version of the following step by step instructions with them, making modifications and adaptation as needed.

1. The following is a way to move into a full breath, called the 3-part breath. The three parts are the abdomen, diaphragm, and chest. You will be focusing on feeling your breath to fill these three areas from abdomen, diaphragm to chest and reversing in the exhale from chest to diaphragm to abdomen.

2. Sit with a straight spine in a chair or in a comfortable seated position on the floor. Ideally you want the hips to be a little higher than the knees so that you can find some length throughout the entire spine especially through your lower back. You don't want to be hunched over and want to be open through the chest.

3. To start rest your palms on your knees facing up and draw your shoulders back and close your eyes if that feels comfortable to you. If you feel more comfortable with your eyes open that is fine too.

4. Feel the sits bones connecting down to the earth and feel the top of the head reaching up to the sky.

5. Then place your hands on your lower-belly and breath in through the nose and let the belly expand into your hands. Breathe out through the nose feeling the belly pull in towards the spine, pushing out the breath. Repeat this, inhale and exhale.

6. Then place your hands on your ribs and we will repeat the same sequence of inhaling and exhaling. Inhale, feeling the ribs and the belly expand. Exhale feeling the belly pull in and drop down with a slow stream of breath. Remember to maintain a tall spine.

7. Then place the hands up to the chest and we will repeat the same sequence of inhaling and exhaling. Breathe into the belly again feeling the breath fill the belly, the ribs, and all the way up to the chest. You can even feel the chest rise. And exhale, pushing out the breath. Repeat. As you inhale and exhale be aware of the three parts of the breath, as it moves in the inhale from abdomen, diaphragm to chest and reversing in the exhale from chest to diaphragm to abdomen. 


\section{ACTIVITY 10: Ocean Breath}

DURATION: 5 minutes modifying to shorter or longer periods as desired.

Objective: Participants will learn to calm the parasympathetic nervous system, decreasing their stress and anxiety. It also helps to bring them into the present moment and into a state of awareness/alertness. It can also build up their energy and internal body heat.
Creative Asset 3. Know how to self-sooth

Creative Asset 9: Know how to establish a safe space for her to be creative

Source: Sophia Mallie, http://www.yogawithsophia.com/ based on Hatha Yoga

Note: Can be done individually or in a group.

\section{Instructions:}

Lead participants through the following exercise. Start by introducing the activity. Then share a version of the following step by step instructions with them, making modifications and adaptation as needed.

About: The ocean breath, or Ujjayi breath (pronounced oo-jai) is translated as "victorious breath." This exercise is sometimes called the ocean breath because it can sound like the waves. It helps when doing this activity to imagine that your throat is like a hose and that you are cramping the hose a little bit so that the water, your breath, flows out a little slower but still with force. The ujjayai breath can be incorporated into exercise and movement including yoga poses or running.

1. Sit with a straight spine in a chair or in a comfortable seated position on the floor. Ideally you want the hips to be a little higher than the knees so that you can find some length throughout the entire spine especially through your lower back. You don't want to be hunched over and want to be open through the chest.

2. Hold one of your arms out in front of you with the palm facing towards you, as if you are looking in a mirror. The other arm can rest comfortably on your knee.

3. Deeply inhale through your nose and exhale opening the mouth as if you are trying to fog up a mirror or your sunglasses. You will actually make a "haaaah" sound. You can think of the Star Wars character Darth Vadar to imitate the sound he makes when he breathes.

4. Inhale deeply again through the nose, and exhale fogging up the mirror. Try to make the exhale last for 5 counts or seconds.

5. Deeply inhale again, and exhale again fogging up the mirror for five counts or seconds.

6. Next, we will repeat this same style of breath but with the mouth closed. At this time, you can lower the hand you have been holding up. You still want to strive to make the ocean sound with the lips closed. With the lips closed you will exhale through the nose and you will feel the throat constrict/close a little bit too.

7. Repeat the ujjaiyi breath as many times as you wish. You can imagine as you breath in a wave building, and as you breath out a wave crashing. 


\section{ACTIVITY 11: 5-4-3-2-1 Relaxation Technique}

DURATION: 10 minutes

Overview: The following technique is very powerful and can be used to relax in stressful situations; to help re-center or reconnect to the present moment; to help fall asleep; to help relieve pain; and to deal with anxiety attacks. This technique can be safely done anywhere and at any time, except while driving or biking, as it often narrows peripheral vision. It is most helpful when done aloud but can be silently as well. It can become more interactive if done in a group and each person participating shares aloud.
Creative Asset 3: Know how to self-sooth

Source: Michele Butot, MSW Self-care sanctuary blog: http://selfcaresanctuary.blogspot.com

Note: Can be done in a group or individually.

Objectives: Participants will learn to self-sooth and reconnect to the present moment.

\section{Instructions:}

Guide participants through the following steps:

1. Begin by finding a relatively comfortable position for your body, and something pleasant to focus your eyes on. Keep your head relatively still. Your eyes can remain open throughout the exercise, unless you have a pleasant urge to close them, or are using the technique to fall asleep.

2. Begin naming aloud 5 sights, 5 sounds, and 5 physical sensations you are aware of. Repeating an item seen, heard or felt is perfectly okay. Count on your fingers if this is helpful.

3. Proceed to 4 sights, 4 sounds, and 4 physical sensations. If you lose count, simply begin again where you think you were... then 3 things, then 2 things... continue counting down and naming until you reach 1 of each category.

4. When you reach 1 sight, 1 sound, and 1 physical sensation, you can choose whether to repeat the whole exercise again to deepen the effect.

5. When you feel finished, take a minute to reorient yourself to the present by counting yourself up through five breaths, becoming increasingly alert at each breath.

6. Repeat the ujjaiyi breath as many times as you wish. You can imagine as you breath in a wave building, and as you breath out a wave crashing. 


\section{ACTIVITY 12: Canvass Bag Decoration/A Personal Safe Space}

DURATION: 3 hours

MATERIALS: Canvass bags, and decorating materials which can include fabric paint, permanent markers, sequins, cloth scraps

TO PREPARE: Research the context to learn about specific patterns, motifs, and/or fabrics that can be introduced as ideas for the participants to use.

Objectives: Participants will enhance their sense of security and safety and will create an object that can carry items that are of importance to them.
Creative Asset 9: Know how to establish a safe space for her to be creative Source: The Van Art Project with contributions from Christina Mallie, Colors of Connection, www.colorsofconnection.org

Overview: Participants decorate canvass bags to transport and/or keep art supplies, art journals or other personal items. In this activity participants construct a literal and a symbolic personal safe space where they can safely express their emotions.

\section{Instructions:}

1. Pass out blank canvass bags to each participant and materials to decorate them with.

2. Explain - "Today, we are going to each decorate our own canvass bag. Each of you gets to choose from the available materials and decide how you want to decorate."

3. Give participants plenty of time to finish the assignment as it can be time-consuming. Sometimes, they may want to take the bag and materials home to finish it there.

4. Participants may need help writing their names on their bags if they decide they want to or are unable to write.

Once everyone is done, they may want to do a fashion parade to show off their new bags.

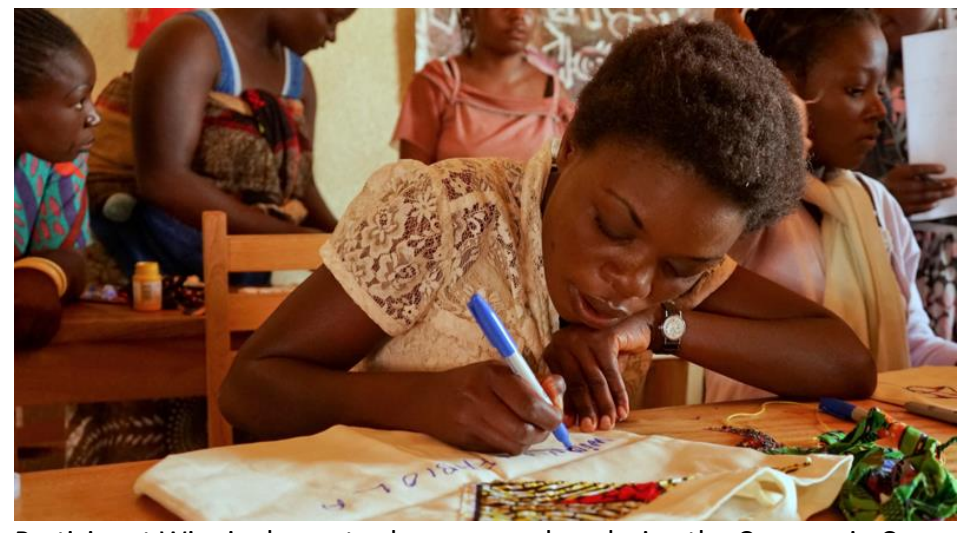

Participant Winnie decorates her canvass bag during the Courage in Congo Project in Goma, DRC, 2015. Photo Credit: Pamela Tulizo.

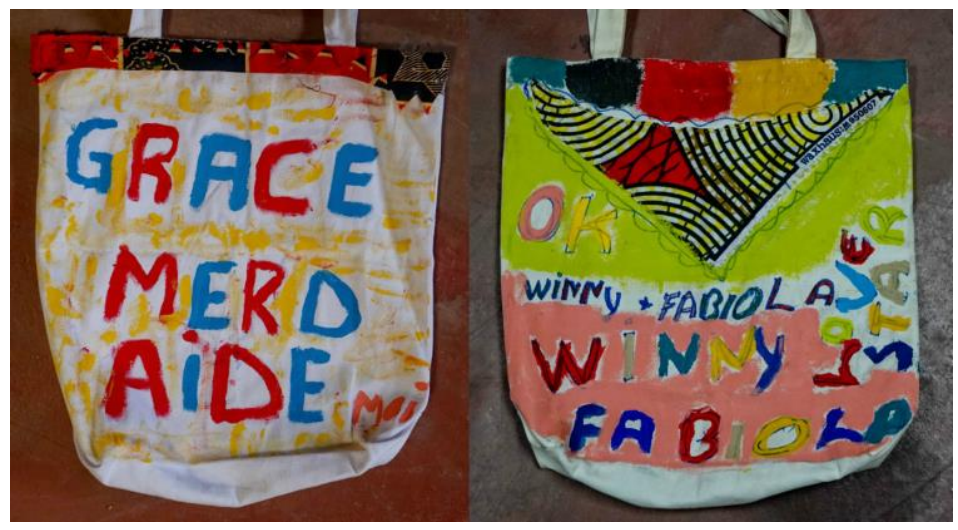

Examples of two bags after activity was completed by participants during the Colors of Connection project Courage in Congo in Goma, DRC, 2015. Photo Credit: Pamela Tulizo. 
DURATION: 30- 45 minutes

MATERIALS: Blank paper, magazines, old photos if available, markers, scissors, and glue sticks.

TO PREPARE: It is helpful if the facilitator provides several examples, that she has personally created, or others have created. Even examples of generally collages can be helpful for the participants to see.

Overview: Participants create collages based on the directive of creating imaginary or memorable safe and calm spaces.
Creative Asset 9: Know how to create a safe space for her to be creative Source: Margarita Tartakovsky, M.S.

\section{http://psychcentral.com/blog/archives/2015/10/19/3-art-therapy-}

\section{techniques-to-deal-with-anxiety/}

Objectives: Participants will reduce their anxiety and promote their sense of safety.

Notes: This activity can relieve distress particularly in situations of distress and displacement.

Adaptation: This activity can be done with drawing instead of using collage elements. In this case the participants would focus on the color choice and types of lines.

\section{Instructions:}

1. Begin by providing several examples, that the facilitator has personally created, or others have created. Even examples of generally collages can be helpfu for the participants to see. As in the case of giving any examples of artwork, it is important to emphasize that there are infinite ways to make a collage and that no collage will look exactly the same, and there is no right or wrong way to make the collage.

2. Ask participants to gather blank paper, magazines, old photos, markers and a glue stick. Instruct them to "Take several deep breaths. Let yourself take a trip down memory lane, remembering any times that you felt ease, safe or pleasant. This might be a location or with a person. If you can't recall a memory, imagine a location or person that would be relaxing and pleasant."

3. Instruct: "Begin looking through your magazines. Cut out images that capture your attention and remind you of the memory or feeling of ease or pleasure. Try to let the images choose you rather than seeking out the 'right' image. That is, pick images that you're drawn to even if they don't make sense or fit in with what you're thinking. Maybe you have "an inner feeling of like or attraction." Maybe you linger longer on this image, whereas you move on quickly with others.

4. Instruct: "Once you have a collection of images, arrange them to create an overall composition of images, which speaks to what it's like to feel safe or at ease."

5. Instruct "After you're done, you can use the image as a reminder of safety and serenity. See if you can imagine yourself in that safe or pleasant place and what it feels like in your body; evoke all your senses to really embody the feeling." 


\section{ACTIVITY 14: Chase the Scribble}

\section{DURATION: 15-30 minutes}

MATERIALS: White paper - the bigger the paper size the better, different colored pencils, crayons, markers or paint

Overview: Participants pair up and take turns leading and following each other in a scribble exercise. By encouraging attunement with others this activity helps participants connect with others in the group and build social support around this common experience.

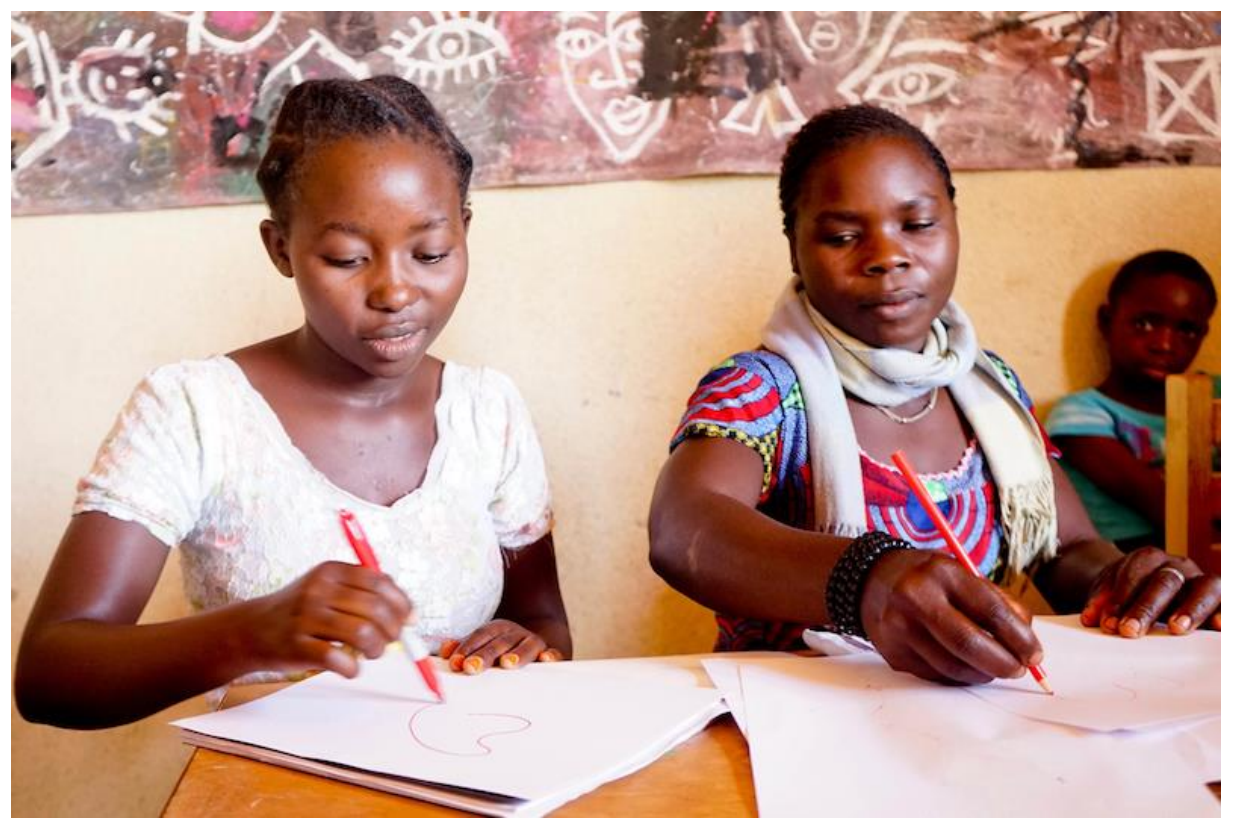

Participant Adele (left) leads in the chase the scribble activity while Esperance (right) follows, Colors of Connection's Courage in Congo Project, Goma, DRC 2015. Photo Credit: Pamela Tulizo.
Creative Asset 3: Know how to self-soothe

Creative Asset 8: Know that she has things in common with others in her community

Source: Cathy Malchiodi, PhD, LPCC, LPAT, ATR-BC, REAT

https://www.cathymalchiodi.com/

Objectives: Participants will calm the mid brain and brain stem through kinesthetic movement, in turn reducing their anxiety, stress and fear and develop connection with others. Participants will regain cognitive function, form attachment and attunement with others, and focus and experience pleasure.

\section{Instructions:}

1. Ask participants to form pairs.

2. Distribute a stack of white paper and two crayons, pencils, markers or paint to each pair. Make sure that the two drawing tools are noticeably different colors for each pair. Explain: Today we are going to engage in an activity called "Chase the Scribble." In this exercise one person will be the leader and one person will be the follower in your pair. You will switch these roles when one person has had a chance to lead several times. The leader will start to draw on the sheet of paper and the follower will draw as close as possible behind. The one rule is that the leader and the follower cannot take the drawing tool off of the page. The scribble you create as the leader can move fast or slow, make many turns and twists as you want.

3. Encourage participants to share what it was like to be a leader and a follower. This can reveal some of the challenges of being a leader and a follower and how to be a more effective leader or follower. Note: If participants are uncomfortable sharing space with each other or the size of the paper is small the pairs can work on separate sheets still mirroring each other and still working with one as the leader and one as the follower. 


\section{ACTIVITY 15: Collaborative Doodle}

\section{DURATION: 45 minutes}

MATERIALS: White paper - the bigger the paper size the better, scissors or razor-blade, different colored pencils, crayons, markers or paint, tape or gluesticks,

TO PREPARE: make photocopies of various doodles and patterns. Include examples of hieroglyphs and runic symbols from different cultures, if possible, to expose participants to different cultures.

Overview: Participants create a collaborative doodle drawing working separating and in groups. By encouraging attunement with others this activity helps participants connect with others in the group and build social support around this common experience.

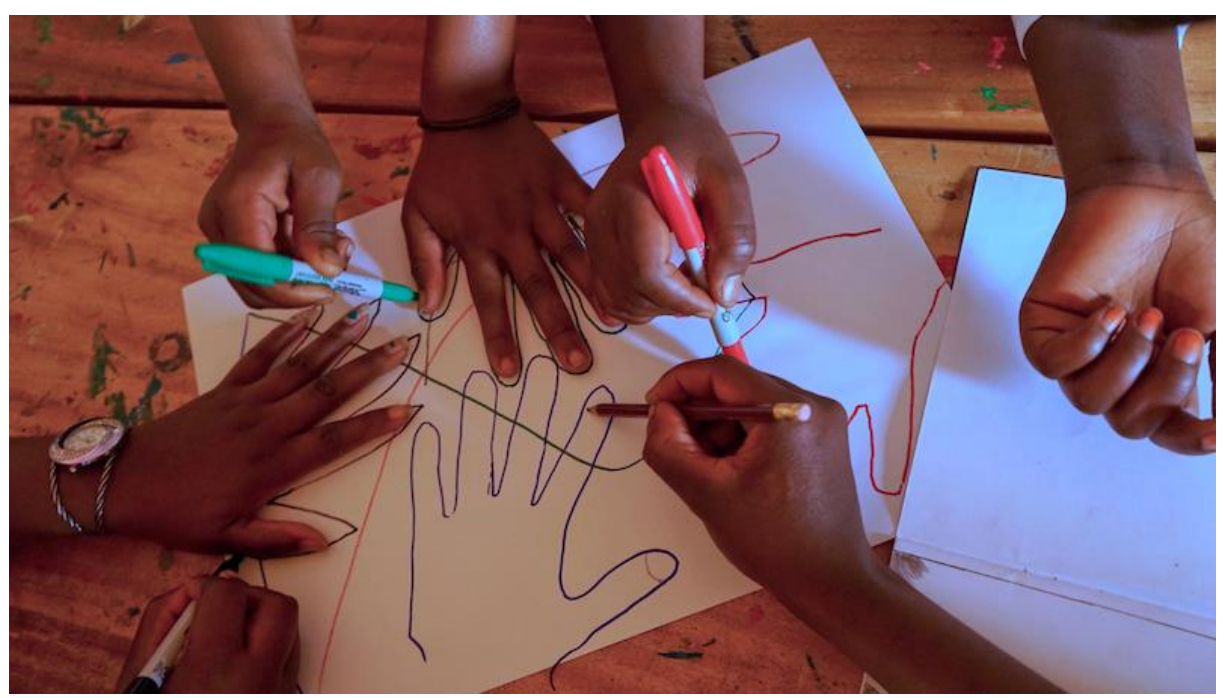

Participants outlining their hands in this activity during Colors of Connection's Courage in Congo Project, Goma, DRC 2015. Photo Credit: Pamela Tulizo.

\section{Instructions:}

1. Ask participants to form groups of 3-5.
Creative Asset 3: Know how to self-soothe

Creative Asset 8: Know that she has things in common with others in her community

Source: Cathy Malchiodi, PhD, LPCC, LPAT, ATR-BC, REAT

https://www.cathymalchiodi.com/

Objectives: Participants will calm the mid brain and brain stem through kinesthetic movement, in turn reducing their anxiety, stress and fear and develop connection with others. Participants will regain cognitive function, form attachment and attunement with others, and focus and experience pleasure.

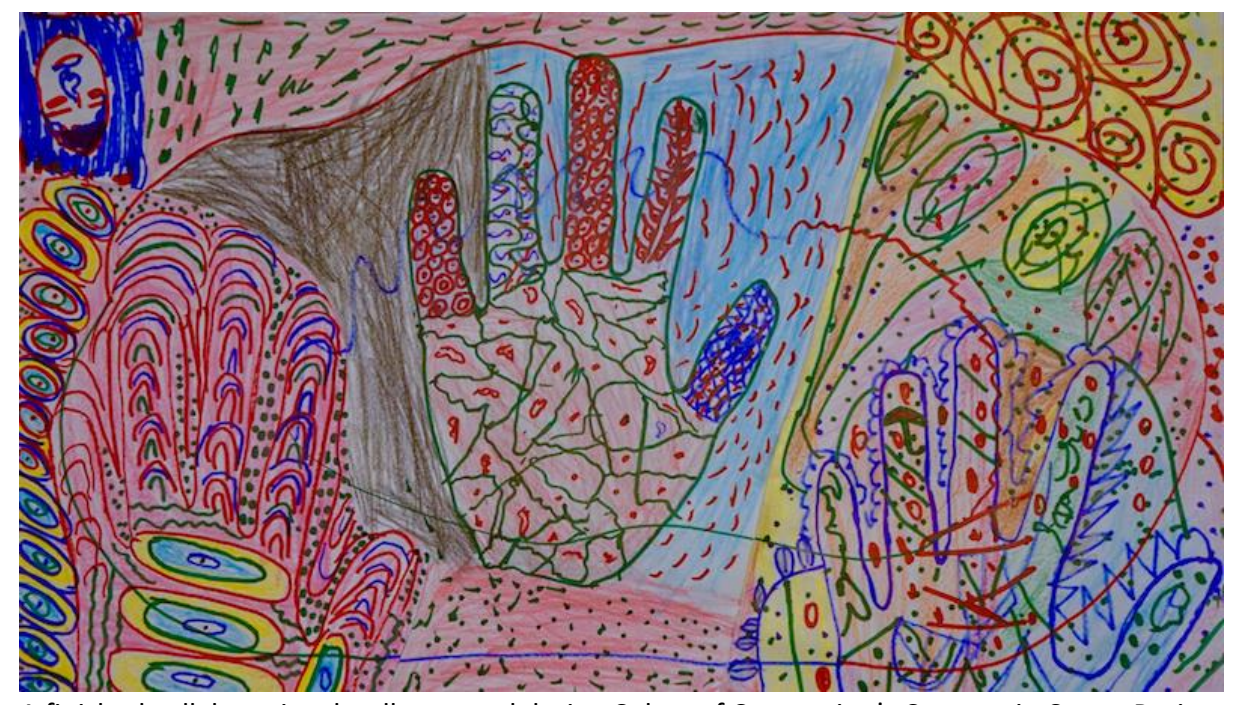

A finished collaborative doodle created during Colors of Connection's Courage in Congo Project, Goma, DRC 2015. Photo Credit: Pamela Tulizo. 
2. Distribute one large sheet of paper and markers, colored pencils, crayons, and/or paint and examples of various doodles and designs that the participants can use as inspiration.

3. Explain: Today we are going to create a collaborative art piece.

4. Ask participants to follow these steps:

a. Each participant contributes to a continuous line/squiggle on the paper that they share with their group. One participant starts, and another picks up where the first left off until each participant has contributed.

b. Each participant places a hand on the paper and outlines it.

c. One participant cuts the paper into as many pieces as there are members in the group. It is not necessary for each piece to be the same size and it is more interesting if the way it is divided does not leave each hand outline intact. The cuts can be zig-zagged, curved or however the participant wishes.

d. Each participant takes a piece of the paper and doodles and colors on it however they want. The existing lines from step 1 and 3 create an existing structure to doodle in.

e. Once all participants have finished decorating their individual pieces each group puts the pieces back together again. Tape or glue stick can be used to reattach the pieces to each other. 


\section{DURATION: 30-45 minutes}

MATERIALS: White paper, different colored pencils, markers or crayons

Overview: Working in pairs, participants create simple abstract drawings and then challenge their partner to use their imagination and creativity to make recognizable things out of these abstract shapes.
Creative Asset 5: Know that making mistakes is an opportunity to learn Source: Adapted by Christina Mallie, Colors of Connection, www.colorsofconnection.org

From the interactive squiggle exercise by Donald Woods Winnicott http://squiggle-foundation.org/

Objectives: Participants will foster playfulness, imagination, creative solutions and relationship building.

Notes: To promote relationship-building between members of the group leave it up to the individuals to choose their partner by moving around the room until they find someone with whom they would like to make contact. To facilitate greater "conversations" between the partners you can ask the participants to exchange drawings one at a time. For example, one person makes 5 lines or shapes on their paper and gives it to the other person who responds by making something out of the 5 lines or shapes. Then their roles switch.

\section{Instructions:}

1. Divide the group into pairs and give each person in the pair a different colored pencil, marker or other drawing tool and a stack of drawing paper.

2. Guide participants through the following steps:

a. Each participant takes their colored pencil or marker and draws five lines on a piece of paper. The lines should not be touching, other than that; they can be any size or shape.

b. Each participant repeats this on five pieces of paper. Encourage the participants to be carefree and impulsive as well as varied in their combinations of the five lines. Giving a time constraint can push participants to be less hesitant to make marks on the paper and become more spontaneous.

c. When each has finished drawing their five lines on five pieces of paper, they exchange their stack with the other participants.

d. Each participant then must work with the five sheets they have received from their partner and use all five lines in the drawing to create a picture or separate things. The only rule is that the original five lines need to still remain visible in their original color when the drawing is completed, and all five lines must be incorporated in the drawing no matter how silly the drawing may turn out to be.

e. Encourage the participants to try to come up with new things each time they are working with a new sheet of paper and set of shapes.

f. Once all the participants have completed the exercise ask them to choose their favorite one out of the five, they worked with and present what they created to the group.

3. Share with participants that sometimes "mistakes" or unexpected shapes can be made into something interesting and valuable. 


\section{DURATION: 60-90 minutes}

MATERIALS: White paper, cut out collage elements (from magazines, newspapers, and/or photo-books, art-books) and glue or clear tape.

TO PREPARE: Pre-cut collage elements pulling from as wide a variety of imagery as possible. Be conscientious of specific cultural references and skin tone of people in the photos and avoid any imagery that could trigger a stressful or traumatic incident (e.g. images of violence). You can laminate the different collage elements to make them more durable and reusable.

Overview: Working with precut collage elements participants select collage elements that defines them. When collages are complete participants present their story to the others in the class. This activity creates visual stimuli that tells a story without a need for prior skill or experience in the arts. When shared, participants recognize their commonalities among their stories and understand how stories can be told visually.

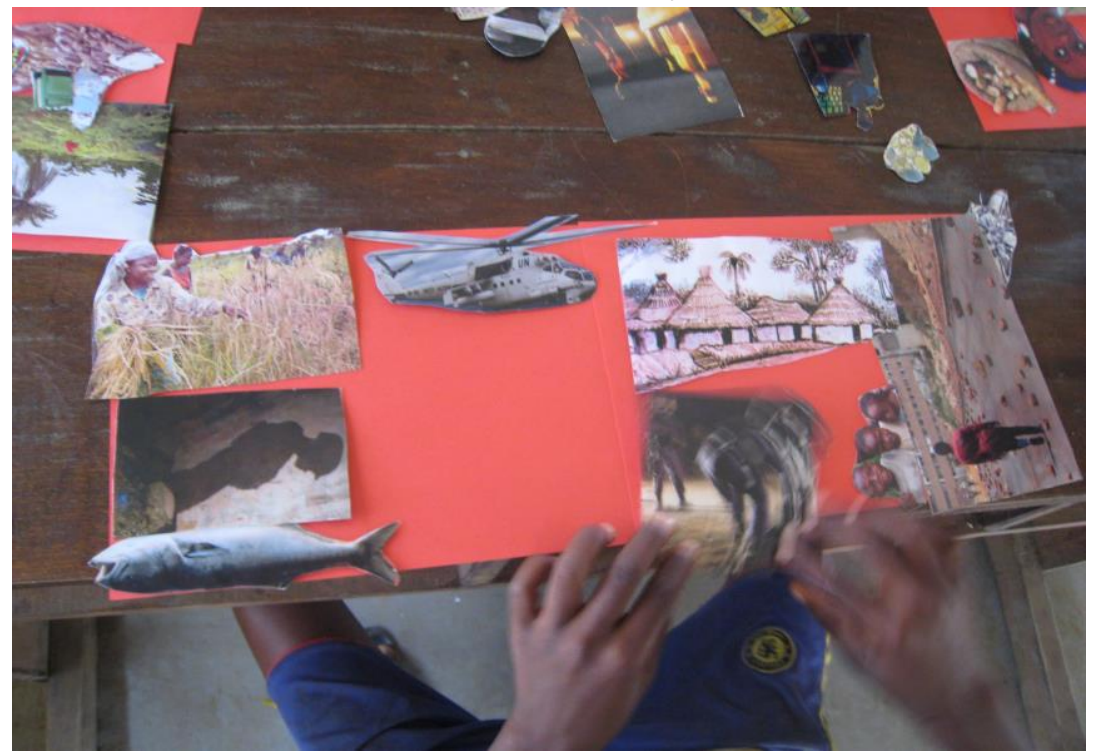

Participant creating a collage during Colors of Connection's project in Little Wlebo Refugee Camp, Maryland County, Liberia 2012. Photo Credit: Christina Mallie.
Creative Asset 4: Know how to gain understanding of herself, her relationships, and her experiences.

Creative Asset 7: Know how to tell a story

Creative Asset 8: Know that she has things in common with others in her community

Creative Asset 9: Know how to establish a safe space for her to be creative

Creative Asset 12: Know that social media and culture often represent women and girls in ways that can be harmful to her health

Creative Asset 13: Be able to identify what beauty means to her

Source: Cathy Malchiodi, PhD, LPCC, LPAT, ATR-BC, REAT

https://www.cathymalchiodi.com/

Bonnie Hirschhorn, LCSW, LCAT, ATR-BC, NCPsyA

http://www.bonniehirschhorn.com/

Objectives: Participants will learn the value of visual storytelling, recognize commonalities with others, and build ability to tell their own stories. Participants will enhance their self-identity and practice public speaking.

Adaptations: This activity is easily adaptable to engage participants in certain subjects. For example, it can be used to explore images of strength and capacity of women, or to compare gender roles in society/portrayal of men and women in advertising.

\section{Instructions:}

1. Spread pre-cut collage elements on a large table or surface.

2. Distribute glue or tape and large white pieces of paper (if a large size is not available improvise by taping two or four smaller sized pieces of paper together.) 
3. Tell participants to select as many collage elements as they wish that define them and can be explained with: I love this, I am this or I feel this.

4. Invite participants to collage the elements to their sheets of paper.

5. Encourage participants to overlap collage elements and experiment with different combinations and compositions.

6. Once the participants have completed their collages have each one share their collage story with the group. Each participant can have a turn to present and to use the language of "I am this, I love this, and/or I feel this" to describe the images on their collage.

7. You can have a general group discussion after everyone has presented to invite reflection on the experience. For example, were you surprised by some of your choices? Did you learn something about yourself or others in this exercise?

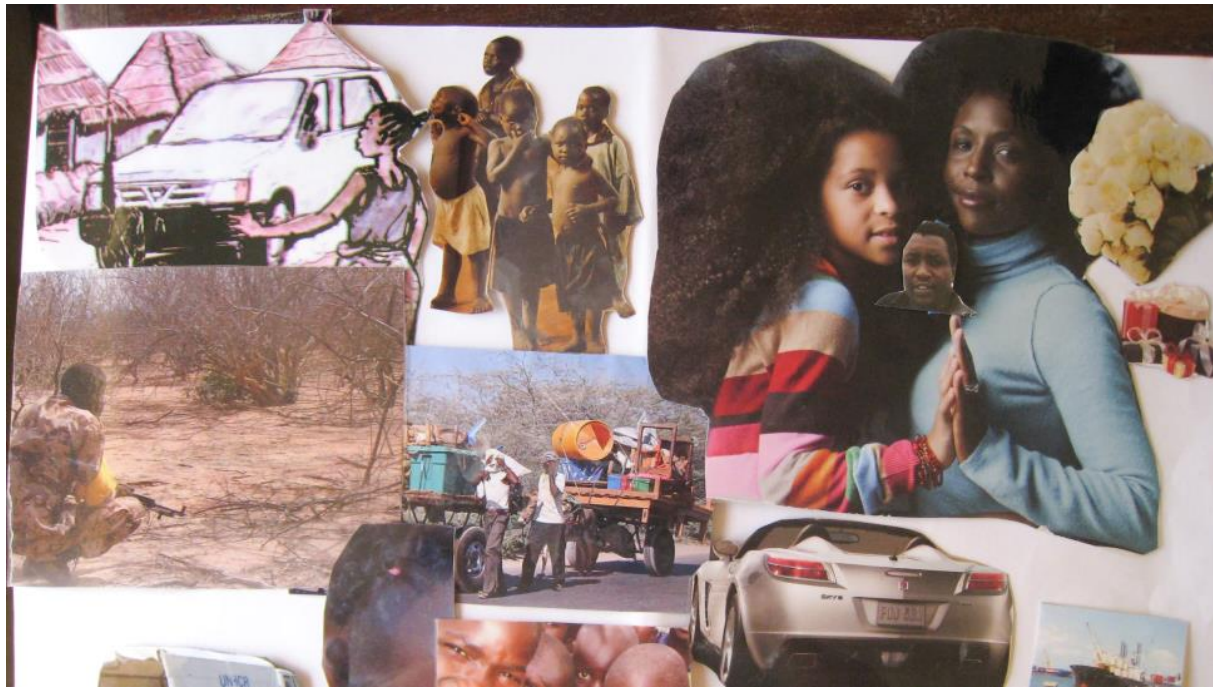

A collage created during Colors of Connection's project in Little Wlebo Refugee Camp, Maryland County, Liberia 2012. Photo Credit: Christina Mallie.

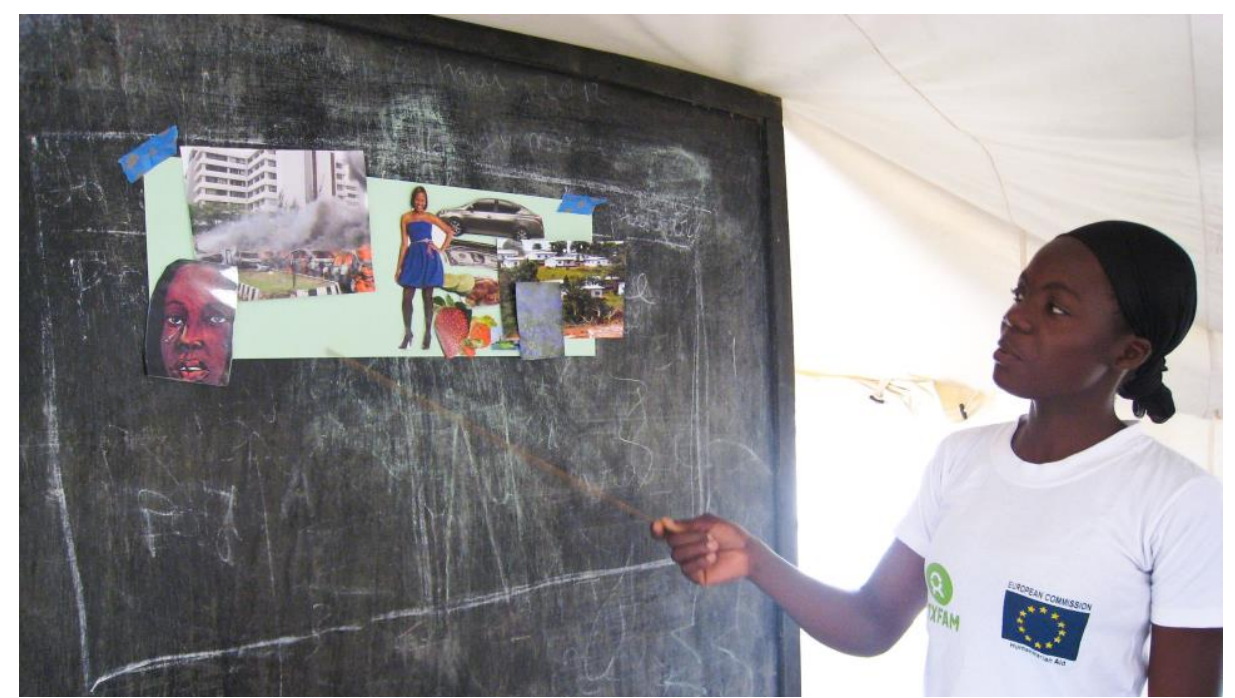

Participant tells the story in her collage during Colors of Connection's project in Little Wlebo Refugee Camp, Maryland County, Liberia 2012. Photo Credit: Christina Mallie 


\section{ACTIVITY 18: Traditional and Non-Traditional Gender Roles Through Photography}

DURATION: 95 minutes

MATERIALS: cameras, batteries, photos that show different composition choices, pro tips sheet (see below)

TO PREPARE: Charge all camera batteries

Overview: Using composition techniques, participants take one picture of a person in a traditional female/male role and one picture of a person in a nontraditional role.
Creative Asset 6: Be able to imagine and express oneself in a different role than what is socially assigned

Source: Babita Patel, KIOO Project, https://kiooproject.org/

Objectives: Participants will understand that gender roles are socially constructed and that both male and females have a capacity and the skills to interchange these roles. Participants will also build appreciation for the distinction between traditional and nontraditional roles.

\section{Instructions:}

1. Thematic Discussion: Gender Roles Traditional and Non-Traditional Gender Roles (15 mins)

Break participants into 2 groups to discuss the following:

- What gender norms do you see for girls/women? (What does society say is feminine?) How does this show up in the roles girls/women play, whether in society, family, or the professional world?

- What gender norms do you see for boys/men? (What does society say is masculine?) How does this show up in the roles boys/men play, whether in society, family, or the professional world?

- Where do you see both genders playing roles that reinforce these norms?

- Where do you see examples of non-traditional roles - roles that men and women can both do without pushing against tradition?

\section{Technique Explanation Composition (15 mins)}

Composition is how you frame a picture. It is the intention of what to INCLUDE and what to EXCLUDE.

- What or who is it you want to take a picture of?

- What is it you want to show/portray about this person to tell who they are? What do you intentionally INCLUDE or EXCLUDE to tell this story?

- Check out the 4 corners. Is everything inside that should be inside / is everything outside that should be outside of the box?

- Activity: Have participants look through set of portraits to find examples of composition techniques. Each participant briefly shares favorite example to the group. Explanation should include what elements in the photograph tells the story of who the person is.

3. Assignment Explanation (5 mins) 
- Using our composition techniques, take one picture of a person in a traditionally gendered role and one picture of a person in a non-traditional role.

- Be prepared to talk about the composition of your picture and how each person represents a traditional or non-traditional role. What was intentionally included and excluded from the photograph to tell who this person is?

- Review norms relating to portraits: Conversing with the subject first to learn who they are and their story. Asking for permission before taking someone's photograph.

- Photo Planning: Give participants 1 minute to think through their photos.

What do they want to take pictures of?

What do they want to show/portray?

How will they frame it?

They may get outside and find other photos they want to take as well, but discuss the importance of thinking through their photographic aims before shooting.

- Pro-Tips See the attached Pro Tips List.

Review previous pro-tips. Add new ones as needed. Reference Pro-tips sheet.

Ask for permission

Optional: Turning on/off flash

Optional: Zoom with your feet

4. Shooting (30 mins)

Keep participants together in pre-approved areas.

5. Transition (5 mins)

Corral participants back to discussion room.

6. Check for Understanding (5 mins)

Pair participants up to discuss their favorite picture and its key composition elements. Pairs discuss the themes in their selected picture, addressing the following:

- Why did you take this picture?

- How does it fit into traditional or non-traditional gender roles?

- Who is it you wanted to take a picture of?

- How did you want to show/portray the person?

- How did you frame it? What did you intentionally include / exclude?

7. Group Discussion (15 mins) 
Allow each participant the chance to answer the question before moving on to the next participant.

- Show us your favorite picture and tell us why this is a traditional or non-traditional gender role.

- What traditional / non-traditional roles do you see for men and women?

- What are your thoughts on these roles?

- How do they make you feel?

- Do you think you saw different kinds of roles based on where we walked around to shoot?

- What if we had chosen a different neighborhood?

- What is one thing you learned today, or one thing you're taking away from our time together thus far?

8. In-Workshop Assignment (5 mins)

- How did you compose your favorite photo?

- What did you intentionally include / exclude and why?

- Did your favorite photo depict a traditional role, or a non-traditional one?

- What are your thoughts on traditional gender roles? How do they frame how you see women \& men? Beyond what you shot today, how might you use photography to convey or show roles or opportunities for young women and men?

\section{Pro Tips}

An evolving list of tips for photographing developed by KIOO Project

Each day, review the previous day's images and offer tips to improve image quality based on what you see. Ex: "Hey, it looks like we got more blur in some of our images than intended last time. If you want to prevent, try..."

Day 1

- Tuck your elbows in

- Hold your camera steady when you hit the button

- Hold your camera button down for a while, do not shake

Day 2

- Ask for permission

- Turning on/off flash

- Zoom with your feet

Day 3 
- Take multiple pictures of object to get the perfect shot

Day 4

- Self-timer: Explain self-timers and how that allows the photographer to set up a bigger scene / story to take a self-portrait. Mirrors and other reflections can be used too.

- Do not share your camera - this is a self-portrait so you must take it on your own!! Others

- Use your body: bend down low, get up high

- Keep the sun behind you 
DURATION: 90 minutes

MATERIALS: cameras, batteries, photos that show a variety of perspectives, and pro-tips sheet see attached.

TO PREPARE: Charge all camera batteries.

Overview: Using the technique of perspective, participants take a self-portrait that conveys their favorite feature of themselves.

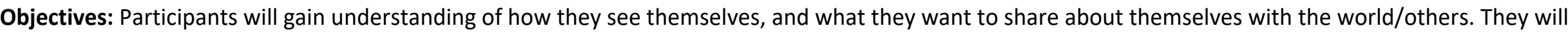
identify aspects of themselves that they appreciate, and think are beautiful.

\section{Instructions:}

1. Thematic Discussion: Being Seen (10 mins)

Guide participants through the following:

Take a few moments to close your eyes and think about your favorite feature - something that makes you YOU. This can be a physical feature or a feature of your personality that you wouldn't be you without it. Maybe this feature allows you to enjoy your favorite things, maybe you wouldn't recognize yourself without it. Today we'll be taking self-portraits that show how you see yourself, and how you want to be seen in the world. Is there a difference between how you see yourself, and how you want to be seen in the world? What about between how you think the world sees you, and who you really are?

2. Technique Explanation (10 mins)

Perspective - Different angles convey different messages:

- From below is called the hero look (In Captain America, the star is shot from below to show he is capable of saving the day.)

- From above is diminutive (In Despicable Me, the kids and the minions are shot from above to convey they are smaller and young.)

Activity: Participants look through class set of photos to identify portraits that show a variety of perspectives.

3. Assignment Explanation (10 mins)

Using the technique of perspective, take a self-portrait that conveys your favorite feature. Again, this can be a physical or personality feature, but your picture must be able to tell the story of you. We ask that you be very intentional when you take the pictures and think about how you compose the picture, 
what you include or DON'T include in the background.

\section{Photo Planning:}

Give participants 1 minute to think through their photos. What do they want to take pictures of? What do they want to show/portray? How will they frame it? They may get outside and find other photos they want to take as well but discuss the importance of thinking through their photographic aims before shooting.

\section{Pro-Tips: See the Pro-Tips list in Activity 19.}

- Review previous pro-tips. Add new ones as needed. Reference Pro-tips sheet.

- Self-timer: Explain self-timers and how that allows the photographer to set up a bigger scene / story to take a self-portrait. Mirrors and other reflections can be used too.

- Do not share your camera - this is a self-portrait so you must take it on your own!! You must hit the button yourself.

4. Shooting (30 mins)

Keep participants together in pre-approved areas.

5. Transition (5 mins)

Corral participants back to discussion room.

6. Pair Share (10 mins)

Pair participants up to discuss their favorite picture. Very briefly reiterate group norms pertaining to sensitive subjects and safe space. The instructors would like to circulate during this activity; if you'd like to maintain privacy with your partner as you share, please raise your hand like this (demonstrate) as we walk past \& we will not listen into your conversation.

- How does your favorite image demonstrate the way that you see yourself?

- What is your favorite feature?

- How did you convey that? Were there other ways you considered conveying it?

- Why did you choose this one?

7. Group Discussion (10 mins)

- Before today, have you ever put much thought into how you want to appear, how you are perceived, and who you really are? If so, did today's assignment change anything about that, or further solidify your perspective? If not, why not?

- Photography is special because you get to choose the stories you tell. How can you use photography, particularly the kind of work we did today, to convey roles or opportunities for men and women?

- What is one thing you learned today, or one thing you're taking away from our time together thus far?

8. In-Workshop Assignment (5 mins)

- How did you use perspective to create your favorite self-portrait from today? 
- Was there anything about yourself that is unique to you that didn't show through in your portraits? If so, what?

Please fill out the Venn diagram with 3-5 attributes each for Who I Am, How I'm Perceived, and How I Want 


\section{ACTIVITY 20: Transformed Photo Self-Portrait}

\section{DURATION: 60 minutes}

MATERIALS: Black and white print outs of each participant's face and as wide a variety as possible of crayons, pencils, paint, sequins, or other craft materials

TO PREPARE: Photograph each participant in a portrait style that focuses on the face. Print out photographs onto regular black and white paper producing a photocopy quality. Black and white is used so that the participants can later add their own color. If possible, lighten the value of the portraits so that there is less black, and the portraits are washed out.

Objectives: Participants will learn to express self-appreciation and acknowledge their own beauty.
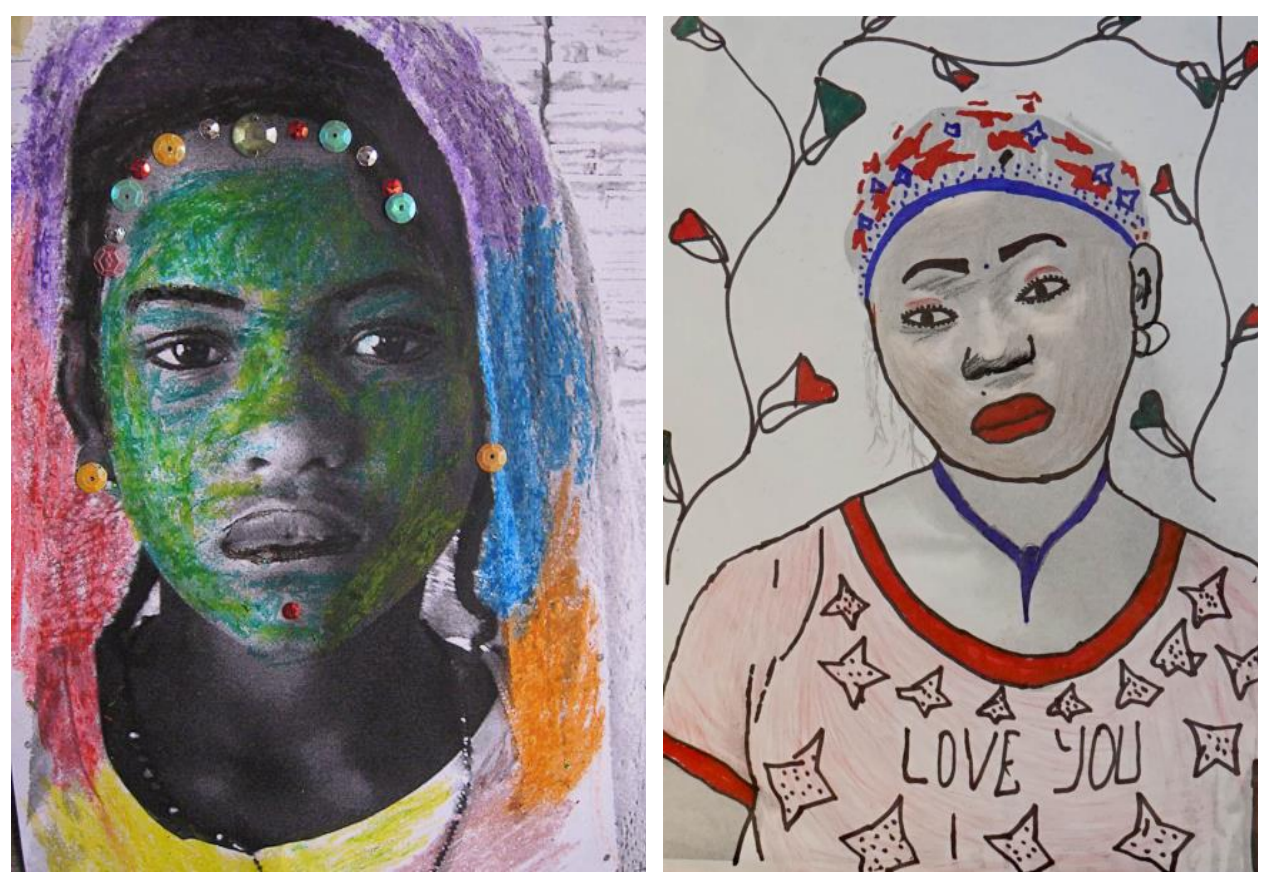

Creative Asset 9: Know how to establish a safe space for her to be creative

Creative Asset 13: Be able to identify what beauty means to her

Source: Megan Brady, ATR-BC, LCPC,

https://urbanwellnesscounseling.com/megan-brady/

Carol McCullough-Dieter, MA-ATC, BHT

Overview: This provides an easy way for participants to create self-portraits without having to worry about being skilled in drawing techniques.

Participants create a self-portrait by transforming a black and white photo of themselves with art and other craft materials.
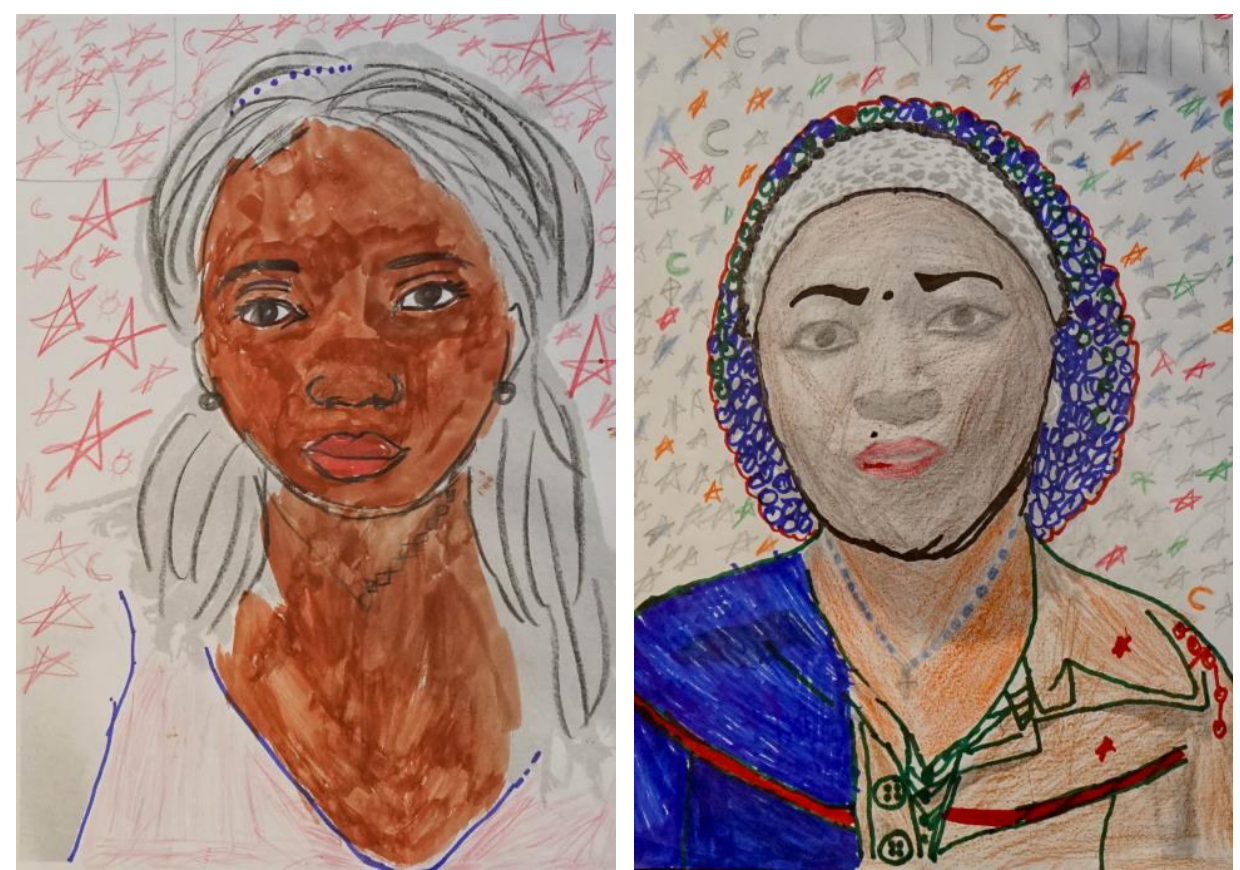

Examples of completed portraits from by participants during Colors of Connection's Courage in Congo Project, Goma, DRC 2015. Photo Credit: Pamela Tulizo. 


\section{Instructions:}

1. Give each participant a black and white print out of her face.

2. Instruct the participant to color over, adorn or decorate the printouts with available materials that can include any arts or crafts materials such as markers, colored pencils, paint or sequins.

3. Give a demonstration beforehand to show different options and styles. Showing multiple approaches is a good idea as otherwise participants may just want to copy the one example.

4. You can introduce elements that express love and beauty such as flowers, hearts, stars, the color gold or silver, or sparkles, and encourage participants to use these to express their appreciation of themselves. You can also encourage participants to invent jewelry, of clothing or add colors that didn't exist in the photo but that they would like to imagine as part of their idea portrait.

5. Some participants may want to write their names on their portraits or other words/phrases and may need help with this if they are illiterate. 


\section{ACTIVITY 21: Telling Your Story, Being Heard}

DURATION: 50 minutes

MATERIALS: Photos taken by participants (either as contact sheets or on camera) and general examples of captioned photos, index cards or similarsized paper, pens or pencils

Note: Part of this activity uses photographs that were taken by the participants themselves on a previous day. If unavailable, one can adapt this this activity by skipping steps $3 \& 4$ as stated. Instead, various images can be provided to the participants to write captions for.
Creative Asset 7: Know how to tell a story

Source: Babita Patel, KIOO Project, https://kiooproject.org/

Overview: Participants caption photographs as a way to tell a story with words.

Objectives: Participants will learn to effectively communicate their feelings, thoughts, and opinions through storytelling.

\section{Instructions:}

1. Thematic Discussion: Being Heard (5 mins)

Guide participants through the following:

- Even when you're seen the way you want to be seen, does that always mean that you're fully understood?

- How many of you have stories of articulating yourself but still not being heard? (show of hands)

- If a complete stranger looked at your favorite photo and described it back to you, what might they miss about who you are?

2. Technique Explanation: Captioning (10 mins)

As photographers, we are storytellers who tell stories through image and captioning is the opportunity to tell even more of the story with words. Captioning allows photographers to:

- Explain more of what is going on in the image

- Provide background information

- Share your feelings or why you shot the image

- Explore what the image means to you

- Describe what inspired you to take the image

- Convey what you were thinking as you shot it

- Reveal what the viewer might not know just by looking at the image

Activity: Split participants into two even-sized groups. Have each member of Group 1 select an image from the class set and stand with it at the front of the room. Members of Group 2 go stand with their favorite image from Group 1, creating pairs. Each member of the pair takes 1 minute to develop different 
stories about their shared image. Ask for a few participants to share their favorite story pairs, highlighting the different ways the same image can be perceived by different eyes.

3. Assignment Explanation ( 5 mins)

Demonstrate the process for using the notecards to caption images.

- Write first name, age and grade on top left-hand side

- Write date on top right-hand side

- Think of a name or title for your image

- Write image name and image number below your name

- Write the caption for the image

4. Captioning (20 mins)

Write a caption for 5 of your favorite images for a total of 5 captions.

5. Group Discussion (10 mins)

- Can anyone share how their caption changed or broadened the story told by one of their photos?

- In what other ways can you use photography to get untold stories out there? What about stories of girls' broadening opportunities - what does that look like in your community? Would it make for some great photos? Who might be affected by seeing those photos?

- What did you learn during the workshop? It can be about photography, something about yourself, your community society or anything else. 


\section{DURATION: $1-3$ hours}

MATERIALS: Colored pencils, black or white paper, and a round plate or compass to make a circle. If possible, use The Mandala Book, a visual symphony, filled with 500 stunning mandala images from nature and civilization.

TO PREPARE: find examples of mandalas including from science/nature, history and art and prepare a presentation of these images for the participants.

Overview: Individuals create mandalas using art materials and/or found objects. The mandala ("sacred circle") is both universal and deeply personal. Creating a sense of order and calm within a sacred, inviolate space, the mandala is considered one of the most effective modalities in treating survivors of sexualized violence.

\section{Instructions:}

Give a general presentation of the mandala to participants including examples from science/nature, history and art. The presentation should utilize several mediums, which give visual examples of the mandala pattern and the Golden Ratio (fractals).

Share any or all of the following information on mandalas depending on how much time you have, the age of the participants taking into consideration their attention span and critical and cognitive skills.

\section{Give the following instructions to create individual mandalas:}

1. Ask participants to take a few moments to meditate and relax.

2. Using colored pencils or oil pastels, graphite pencil and eraser, ruler, paper, and a round plate or compass to make a circle. Try a circle about 10 inches in diameter, but you can use any size paper to make your drawing. White
Creative Asset 2: Know how to express feelings with and without words

Creative Asset 3: Know how to self-sooth

Creative Asset 4: Know how to gain understanding of herself, her relationships, and her experiences.

Creative Asset 9: Know how to establish a safe space for her to be creative

Sources: Cathy Malchiodi, PhD, LPCC, LPAT, ATR-BC, REAT

https://www.cathymalchiodi.com/

Lori Bailey Cunningham http://www.mandalaproject.org/About/bailey.html

Dr. Judith Cornell, PhD, and Suchinta Abhayaratna, PhD

http://www.chisuchinta.com/

Objectives: Girls will have increased sense of calm and safety.

\section{Information about Mandalas:}

We humans have always had a fascination with the circle. We experience it throughout nature - in the spiral of the Milky Way, the orbiting planets, and the cycles of life itself. As children, we also discover that we can use a crayon to make circular forms on paper; it's a universal stage of artistic development that every normal child throughout the world experiences. In fact, it is the first major milestone in image-making and for that reason, a child's circle drawing may be one the earliest representations of the self. Circular forms in art are often referred to as mandalas, the Sanskrit word for "sacred circle." For thousands of years the creation of circular, often geometric designs has been part of spiritual practices around the world and almost every culture has revered the power of the circle. Eastern cultures have used specific mandalas for visual meditation for many centuries; the Tibetan Buddhist Kalachakra, also known as the Wheel of Time, is probably one of the most famous mandalas and symbolically illustrates the entire structure of the universe. 
paper is fine, but also try a sheet of black paper, too. It will make the colors "pop" because of the darker background. Explore whatever shapes, colors, patterns or recognizable things they feel connected to and these could be geometric or natural. The one guideline is that the mandala has one identifiable center point from which all else emanates. ișpi Notice the images that appear in your mind. Let the image take on a life of its own. Fill in the entire circle.

3. Because mandala drawing can be a very relaxing and meditative experience, you might want to play some soft instrumental music to set the mood.

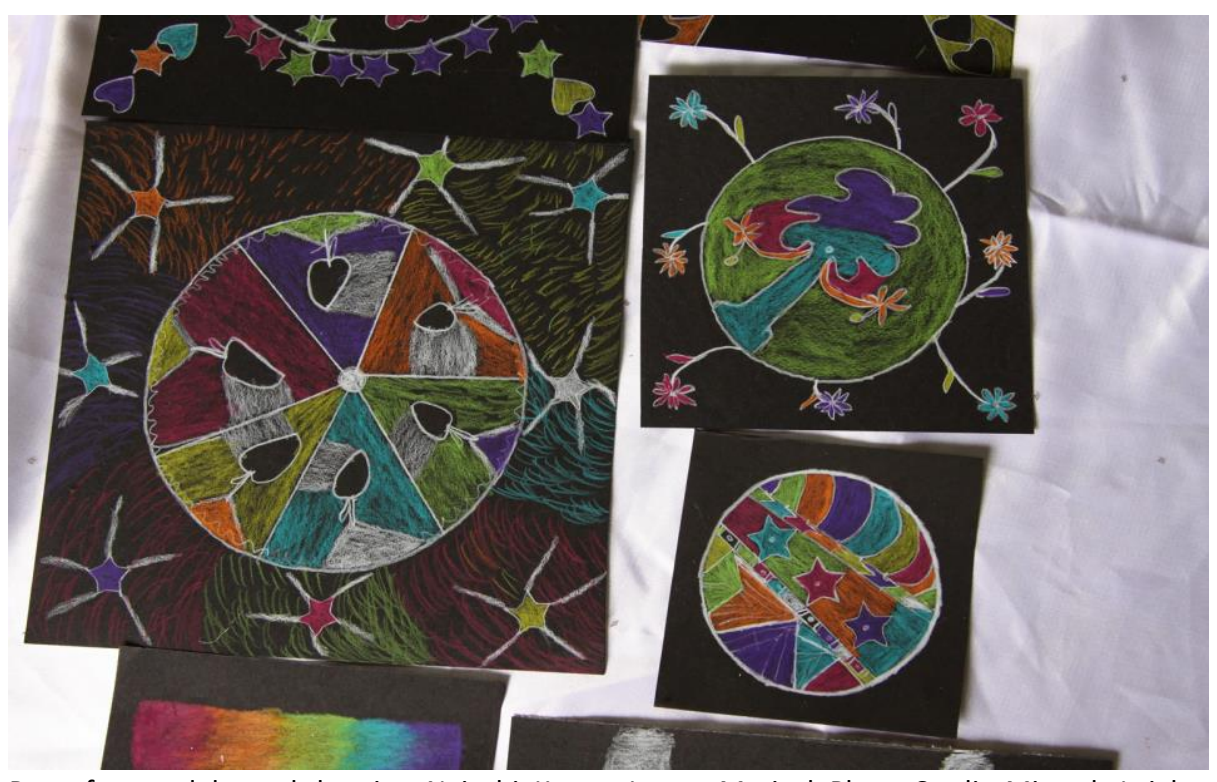

Part of a mandela workshop in a Nairobi, Kenya, Joyaux Marisol. Photo Credit: Miranda Leigh.
Circular forms are found at the prehistoric Stonehenge monument in England and the 13th century labyrinth at the base of Chartres Cathedral in France. Spiritual seekers have consistently created mandalas to bring forth the sacred through images and have evoked the circle in ritual and art making for the purpose of transcendence, mindfulness and wellness.

Carl Gustav Jung is credited with introducing the Eastern concept of the mandala to Western thought and believed this symbol represented the total personality - aka the Self. Jung noted that when a mandala image suddenly turned up in dreams or art, it was usually an indication of movement toward a new self-knowledge. He observed that his patients often spontaneously created circle drawings and had his own profound personal experience with mandala images. From 1916 through 1920, Jung created mandala paintings and sketches that he felt corresponded to his inner situation at the time. He believed that mandalas denoted a unification of opposites, served as expressions of the self, and represented the sum of who we are. 
DURATION: Varies depending on the size of the danmala, types of materials used and size of the group

MATERIALS: Variety of local, readily available materials either from nature or man-made. If possible, use The Mandala Book, a visual symphony, filled with 500 stunning mandala images from nature and civilization

TO PREPARE: find examples of danmalas and prepare a presentation of these images for the participants.

Overview: A group collectively creates a danmala made from a variety of materials

Objectives: Participants will cultivate an understanding of unity, strength in numbers, an awareness of the power of many, and the ability for a diverse group of individuals to create something beautiful together.

\section{Instructions:}

1. Give a general presentation of the danmala and mandala which includes examples from science/nature, history and art. You can emphasize that this form exists across many cultures.

2. Either provide an array of materials or have the participants gather different materials to use. Emphasize that materials can be almost anything as long as you are able to have multiples. Examples of readily available materials could be bottle caps, leaves, sticks, flowers, or rice. If participants select objects from their home or own environment this can bring more significance to the danmala as it will reflect something of their personal lives.

3. Choose a location either outside or inside, public or private that the group collectively decides upon as an ideal space to create the danmala. When choosing the location take into consideration whether it is a space that the danmala can be installed in for a period of time, whether it will be exposed
Creative Asset 2: Know how to express feelings with and without words

Creative Asset 3: Know how to self-soothe

Creative Asset 4: Know how to gain understanding of herself, her relationships, and her experiences

Creative Asset 8: Know that she has things in common with others in her community

Creative Asset 10: Know how to establish a safe space for a group

Source: Miranda Leigh, Joyaux Marisol, https://www.joyauxmarisol.com/

Notes: There may be opportunities to apply the danmala designs to handcrafted goods that the participants can make and sell locally.

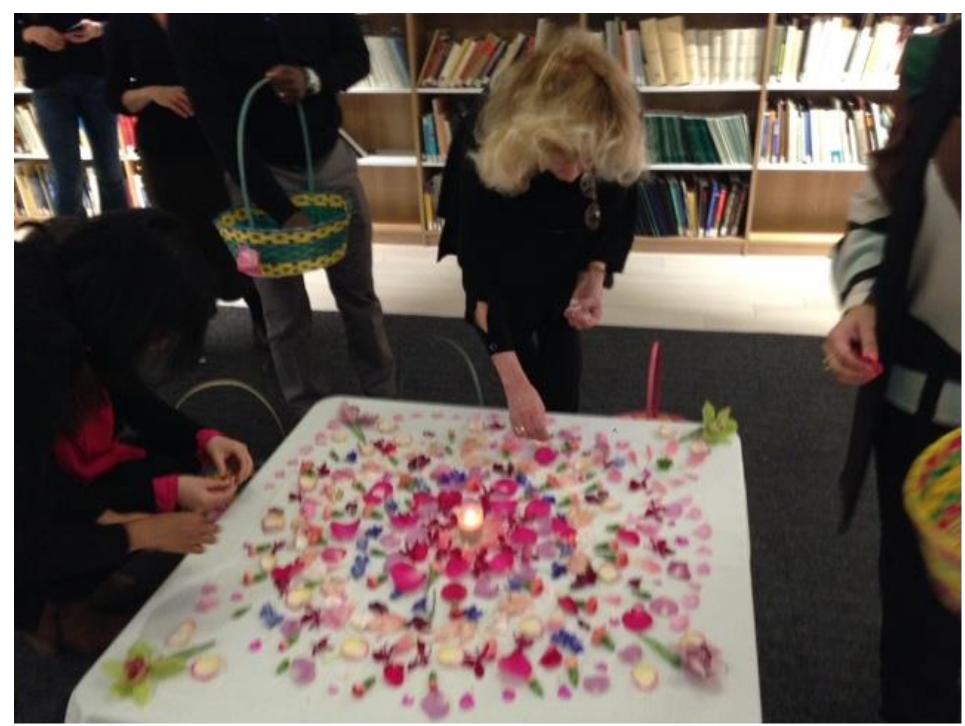

Population Council staff and AGCN colleagues use flower petals, candles, and other objects to assemble a danmala. Photo Credit: Sophie Soares. 
to the elements or people that might disturb it, if it is important for the danmala to be seen by the community or if it is important that it is created in a more private space.

4. Have the group take turns creating the danmala, starting from the center, each participant can add an element. Elements can be created in repeat patterns to establish symmetry or can be asymmetrical.

Once the process is complete ask the participants how it felt to create something together.

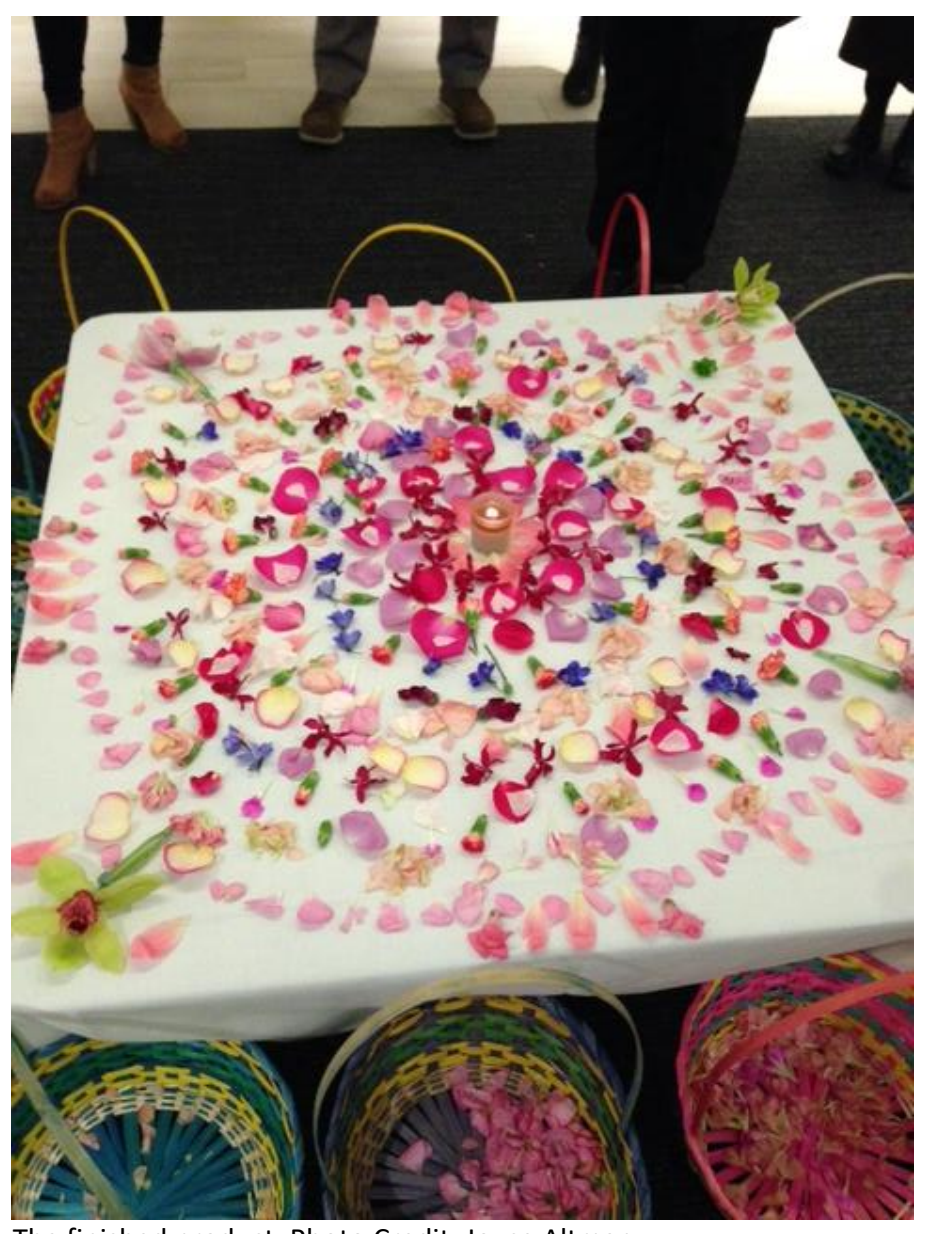

The finished product. Photo Credit: Joyce Altman 


\section{ACTIVITY 24: Goal Setting Using Body Maps}

DURATION: $60-90$ minutes

\section{MATERIALS:}

For Instructor:

- Visualization Text

- Sample of a pre-cut body map silhouette

- Samples of two personal body maps

For Participants:

- Markers

- Scissors

- Butcher Paper

- Journal

TO PREPARE: Practice of the basic partner postures

Objectives: Girls envision their future goals and identify and articulate which ones they would like to pursue and why.

Notes: In this lesson the girls will take part in a creative exercise that will enable them to visualize their goals. Depending on the age of your participants the visualization can be adapted. For girls above the age of twelve, as you guide them through past experiences you can focus on past achievements. Even small achievements can be highlighted and supported.

If you have participants who are illiterate ask them to draw pictures into their body maps instead of using words. For example, if it is someone's goal to go to school possibly she will draw a picture of a book.

A suitable following lesson would be one that supports the girls to identify the steps needed to reach their individual goals.
Creative Asset 1: Know how to identify emotions in herself and in others

Creative Asset 2: Know how to express feelings with and without words

Creative Asset 3: Know how to self-soothe

Creative Asset 4: Know how to gain understanding of herself, her relationships and her experiences

Creative Asset 6: Be able to imagine and express oneself in a different role than what is socially assigned

Creative Asset 13: Be able to identify what beauty means to her

Source: Elana Haviv, Human Rights http://www.generationhumanrights.org/

Overview: The body map activity supports the girls to explore and visualize their goals.

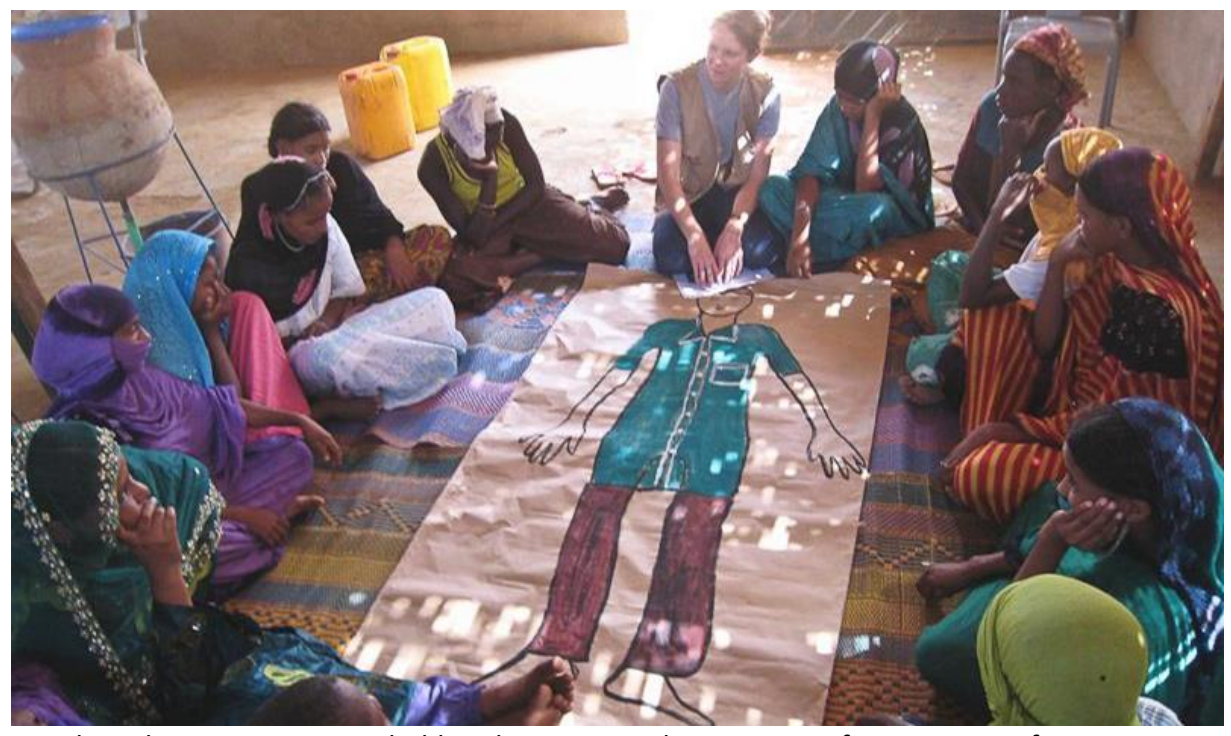

Social Worker Laurie Reyman holds a discussion with one group of participants after

they completed a body mapping exercise. Goudoubo Refugee Camp, Burkina Faso, 2014, Colors of Connection. Photo Credit: Christina Mallie. 
Space Set-Up: Set up the learning space before the girls arrive. Move any furniture to the one side of the room and lay out strips of butcher paper on the floor. When the girls enter have them remain standing up.

\section{Instructions:}

Introduction to Body Maps (5 Min.)

- Share with the girls that in today's lesson they will explore where they have been in their lives and where they want to go. Specifically, what goals they may have for the future.

- Explain that they will create a map of themselves and their personal life journey (past events and future wishes). Show an example of a completed personal body map.

- Describe that they will use their imagination, movement, and visualization to explore their thoughts and vision of their future.

- Let them know that visualization helps us to focus and to relax and is a good stress management technique.

- Encourage them to try everything even if certain parts of the activity seem 'silly' or 'foolish', and to be respectful to their peers.

Body Map Activity Set-Up (20 Min.)

- Show the girls a blank body map silhouette.

- Have them choose a partner and take turns lying down on the butcher paper while the other partner traces her body. Then switch so there will be two separate silhouettes and cut them out.

- Make space for each girl and their body map and give them markers.

Activity: Visualization and Writing/Drawing Body Maps (20 Min.)

Lead the girls through the following visualization and writing exercise. Start by asking them to walk around the room as slowly as they can.

o Feet:

Move around the room very slowly and feel your feet each time they touch the ground. As you walk slowly make sure you do not bump into anyone. Where do your feet take you? Where have they traveled? Have they taken you to new towns? What lands have they walked on? What rooms have they walked in? Who have your feet taken you to? Which people? What places? What events? Was there a place you dreamed of going that your feet took you to?

Where do you want your feet to take you in your life? Where will they lead you in the future? Imagine where you would like your feet to take you in the future. A certain town or city? University? A job you would like to have? A home you would like to own?

When you see at least one place clearly in your mind go back to your body and sit by your feet (on the map). Write or draw into your feet where you would like them to take you in the future. 
When you have finished writing into your feet put your marker down and take a moment look at your hands. Put them in front of you and rub them vigorously together and create some energy. Now look at them again. What things have your hands touched today? People? Food? Water faucets? Did they touch anything hot- maybe too hot? Did they touch another person? Have they helped you to finish an assignment or a project? Have they helped you to cook or take care of a younger sibling?

Think about different things your hands touch throughout your day. Door knobs? What doors have they opened for you? Doors from the past and doors in the present. What doors would you like your hands to open for you in the future? Doors to a classroom? Doors to a job? Or maybe your hands are holding a book you have written? Or maybe your hands will hold a baby?

When you clearly see a door your hand is opening, or something your hands are holding, take out your marker and write or draw what you see into your hands on your body map.

o Brain:

Sit down next to your head on your body map. Place your hands on your head. Imagine traveling into your brain. This is the command center of your body. Your brain is always very busy and filled with countless thoughts. Watch your brain working and try to observe all your thoughts that are passing through it.

If your brain could share with you one plan it has about your future, what would it tell you? Take a moment and keep your hands on your head and wait for an answer. It may be plans for tomorrow or it may be plans for several years from now. Listen to the response.

When you hear the response write or draw your brain's plan for you in your head space on your body map.

o Heart:

Now make your right hand into a fist and place it on the left side of your chest over your heart. Your fist is actually a very close size to your heart. Close your eyes for a moment or gaze at the floor. Imagine you can see your heart pumping blood throughout your body. Imagine you can hear it beating. As you go through your day your heart is always working for you. Now imagine going deeper into your heart. Are there colors? Are there feelings? It is said that we carry stories in our hearts. Does your heart have a story of your future? Find your heart's future story of you.

When you see your heart's story of your future write or draw it into your heart space on your body map.

Check-in with the girls (5-10 Min.)

Entry point questions include:

- What was it like to create your personal geography in the form of a body map?

o Did you remember a vision you had for the for the future that you had forgotten? 
o Did you envision a part of your future that you had not seen or thought of before today?

Journal Writing Drawing (10-15 Min.)

Share with the girls that it is now time to review our body maps and identify one to two goals or future visions that they have written or drawn about themselves.

- Pass out the journals.

- Ask the girls to look at their body maps and identify any visions of themselves they wrote down for their future.

- Entry point questions include:

- Did your feet carry you to a place you would like to work?

- Did your hands hold a cell phone or computer to repair? Or did your hands hold a surgeon's tool? Or possibly carry a baby?)

- Explain to them that these future visions can be goals that they set for themselves.

- Ask the girls to write or draw one to two goals in their journal.

- They can write the goal in a one or two words (for example: teacher, doctor, student, cell phone repair technician, mechanic, etc.), or Draw a picture of their goal(s).

Sharing (Timing to be determined by Instructor)

Take a few minutes to ask if any of the girls would like to share their body maps.

Closing (Until end of class)

- Remind the girls what they learned in this lesson:

o They were courageous and explored the vision of their futures through creative means.

o They identified goals they want to successfully reach.

o They shared goals in words and pictures. 


\section{ACTIVITY 25: Stronger Together Collaborative Work}

DURATION: Varies

\section{MATERIALS: Varies}

Overview: Using individual tiles, individual crochet squares or quilt blocks, each participant creates an individual piece and then all the pieces are put together
Creative Asset 7: Know how to tell a story

Creative Asset 10: Know how to establish a safe space for a group

Source: Bonnie Hirschhorn, LCSW, LCAT, ATR-BC, NCPsyA

http://www.bonniehirschhorn.com/

Objectives: Participants will build connection between group members, establishing a safe space and reinforcing the sense that each individual is stronger together as a group.

\section{Instructions:}

1. Facilitator introduces the intention of the activity which is to recognize how we become stronger through solidarity and togetherness.

2. Depending on the material you choose, have each individual participant create theirs in a session or as a take home activity. For example, if you are working with tiles each participant can decorate their tile with ceramic paint. If you are working with yarn, or fabric, each participant can choose a color or pattern and knit, crochet or sew a square.

3. When each participant has created their individual piece, the activity is completed by connecting all of the piece together.

4. Facilitate a discussion with the participants asking them to share their experience of participating in this activity.

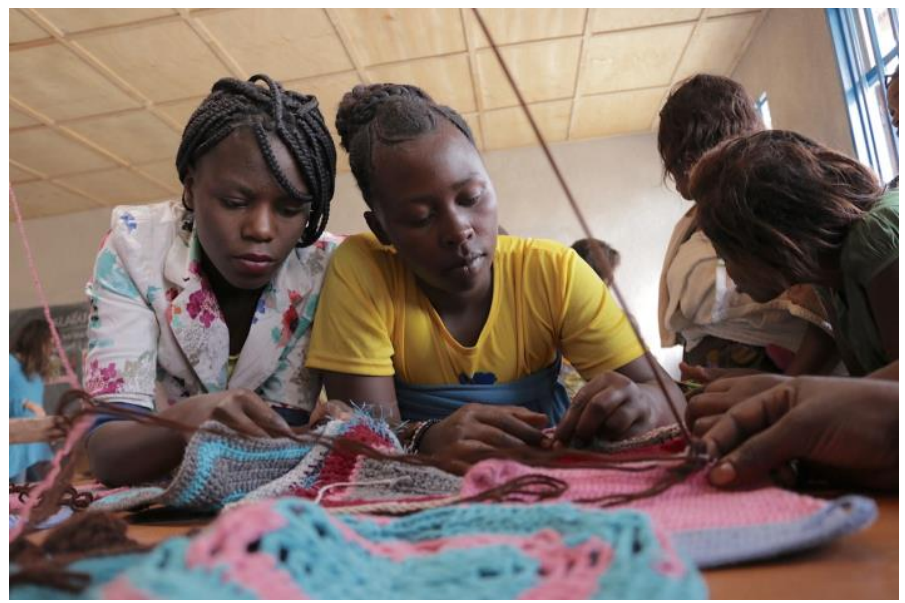

Participants sewing together individual crochet squares for the Strong Together activity during Colors of Connection's Tunaweza Portraits Project, Goma, DRC 2019. Photo Credit: Bernadette Vivuya. 


\section{ACTIVITY 26: Exploring Non-Traditional Gender Roles Through Drawing}

\section{DURATION: 30-45 minutes}

MATERIALS: Paper, and colored pencils or crayons

Objectives: Participants will build their capacity to themselves in nontraditional gender roles.

\section{Instructions:}

1. Ask participants to think about different jobs and then ask in their opinion if usually men or women occupy these positions. Encourage participants to think of all sorts of jobs, including high level ones like the president of a country, or more traditionally male dominated professions like construction workers. When you have a good list of 10-15 different roles, ask participants to identify which roles men usually occupy which that they think women are also capable of doing. Hopefully you will have about 5 examples. During this discussion you can challenge and push participants to imagine themselves, or other women or girls in certain roles. For example, if participants are reluctant to say that a woman can be president give them examples of contemporary women presidents to show them that it is possible.

2. Ask participants to pick one of the 5 examples to draw. They can choose to draw themselves in one of the professions that they think they would personally like to pursue, or they can create a fictional woman or girl in this role. Encourage them to include details, like what the woman would be wearing and something specific that she would be doing in this role.

3. When participants have completed their drawings display them together and ask for participants to give positive comments on each other's drawings.
Creative Asset 6: Be able to imagine and express oneself in a different role than what is socially assigned

Overview: Participants create portraits of women and girls in professions that women don't usually occupy

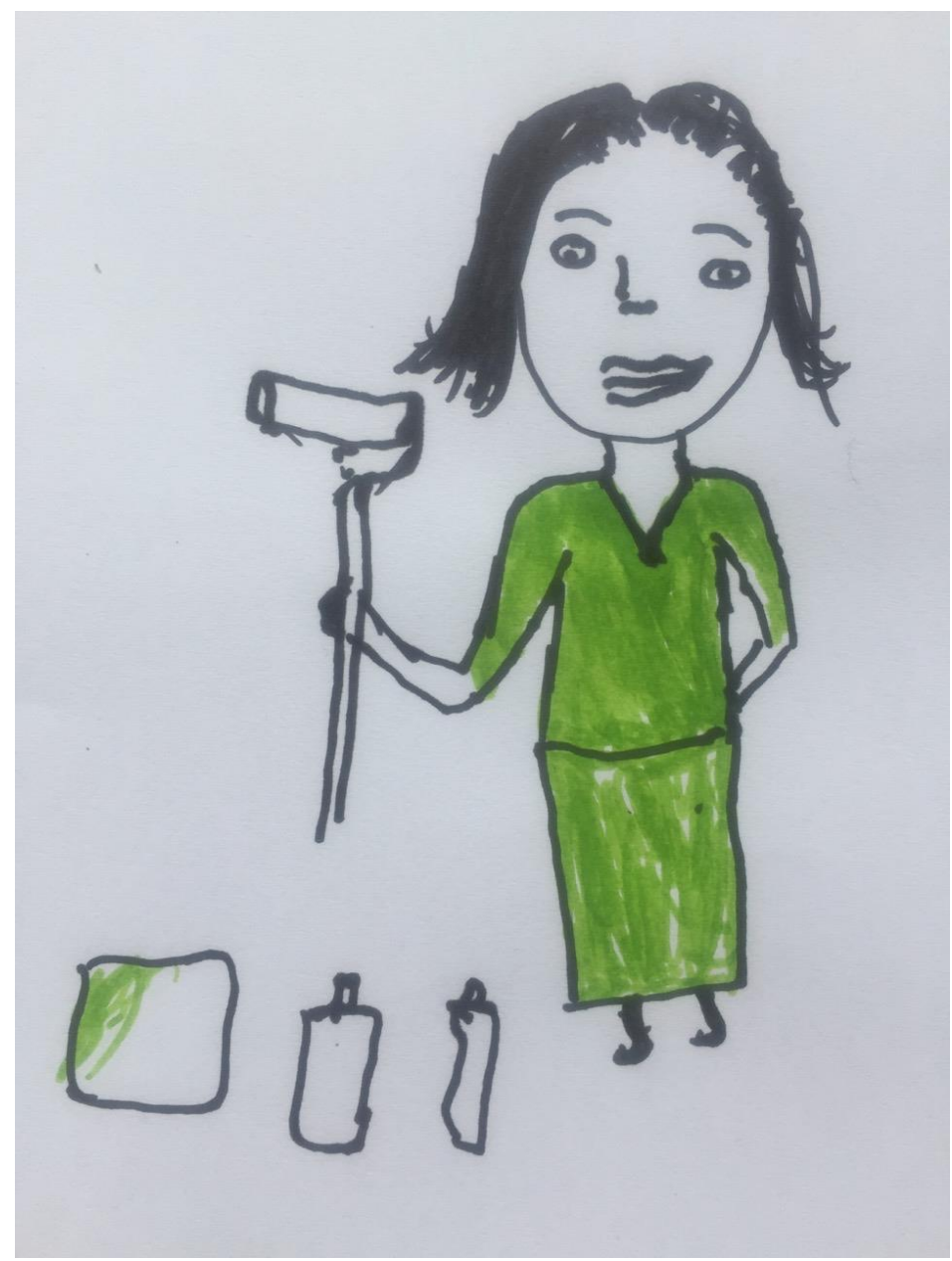

One participant's drawing of a woman painter created during Colors of Connection's Tunaweza Portraits Project, Goma, DRC 2019. Photo Credit: Bernadette Vivuya 


\section{ACTIVITY 27: Musical Emotions}

DURATION: 30 minutes (or shorter depending on the group size)

MATERIALS: Plastic cup, paper, music

TO PREPARE: Create a list of emotions on a piece of paper. Cut the paper so that each emotion is on its own piece. See note for adapting this activity to illiterate populations.

Objectives: Girls will learn to create a safe space that gives them an opportunity to discuss their emotional feelings in a creative musical atmosphere. Girls will build relationships within the group.
Creative Asset 1: Know how to identify emotions in herself and others Creative Asset 2: Know how to express feelings with and without words Creative Asset 8: Know that she has things in common with others in her community

\section{Source: Connor Patterson, Chords of Peace,}

https://www.facebook.com/chordsofpeace/

Note: This activity can be adapted to illiterate populations by having the facilitator write everything down and read to the participant the emotion they select.

\section{Instructions}

1. Have participants brainstorm and create a list of emotions that they have felt throughout their life.

2. Write these emotions on a piece of paper and cut them up into small pieces.

3. Fill a plastic cup with the pieces of paper with the emotions listed on them.

4. Have the participants form a circle and sit down.

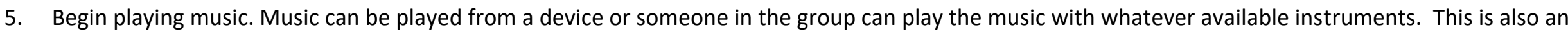
option for participants who choose not to partake in the activity to be responsible for playing the music.

6. Begin passing the cup around while the music is playing.

7. Once the music stops, the participant with the cup in their hand must pull out a piece of paper.

8. The participant acts out the emotion that is written on the paper without speaking or getting up.

9. Participants guess the emotion that is being acted out.

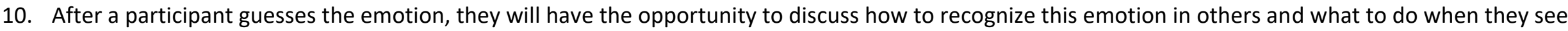
someone showing signs of that emotion. 


\section{ACTIVITY 28: Controlled Expression of Thoughts and Feelings through Improvisation}

DURATION: 30 minutes (or shorter depending on the group size)

MATERIALS: buckets, drum sticks, egg shakers, clave

Objectives: Girls will learn to create musical improvisation in which they freely express themselves, creating a safe and comfortable atmosphere. Girls will engage in and encourage their and others' spontaneity, and foster connection between members of the group.
Creative Asset: 2: Know how to express feelings with and without words Creative Asset 8: Know that she has things in common with others in her community

Source: Adapted by Chords of Peace, from Jaap Orth (2005). "Music Therapy with Traumatized Refugees in a Clinical Setting."

https://doi.org/10.15845/voices.v5i2.227

\section{Instructions:}

1. There are three roles that need to be filled.

a. The facilitator: Uses clave to create a uniform beat.

b.A participant who volunteers to improvise on the bucket drum.

c. The rest of the group is given egg shakers to maintain the tempo.

2. The facilitator asks the participant who has the bucket drum to begin playing whatever comes to mind (There is a strong correlation between a participant's mood and the music that is created).

3. The facilitator then joins the participant by creating a shadow beat on the clave to encourage the participant.

4. The reminder of the group then begins playing their egg shakers to maintain the tempo.

5. Repetition of beats and sounds created by the participant creates a safe structure that is familiar to the participant.

6. At this point in time, the facilitator will ask if the participant would like to sing or speak about the emotions that are being conjured during their playing.

a. The facilitator will ask the participant to continue as long as he/she wishes.

7. When the participant has finished, the facilitator will ask the participant what he/she sang/talked about, and what was felt.

a. This typically empowers others to volunteer as the group becomes more comfortable and understanding that a safe space has been created.

8. A new participant will take over the bucket drumming and the process is repeated. 


\section{ACTIVITY 29: Finding Stories in Songs}

DURATION: 30 minutes (variable depending on how many participants are sharing)

TO PREPARE: Find examples of songs participants are familiar with that tell a story

Objective: Participants will learn to consider new ideas, gain insights on common life experiences, practice active participation, and enhance their listening skills.

\section{Creative Asset 7: Know how to tell a story}

Creative Asset 8: Know that she has things in common with others in her community

Source: Developed by storyteller/author Heather Forest for her storytelling workshops with participants, teachers, and librarians.

http://www.heatherforest.com/

\section{Instructions:}

1. Find and learn songs which tell a story. Notes that folk ballads and contemporary songs often suggest a larger tale.

2. With the group of participants listen to the chosen songs and then ask participants to retell the story in the song in their own words. Or have a "storysong" concert which participants perform their songs. 
DURATION: 30 minutes

MATERIALS: Wrapper cloth (e.g. lappa, kanga, kikwembe, or payne)

Overview: In this activity each participant is invited into the center of the circle to present something they can do with the wrapper. The only rule is that everyone has to present something different.

Objectives: Participants will foster connection and enhance their storytelling and expressive abilities.

Note: This activity was developed in the sub-Saharan African context, so may need to be adapted for other contexts.

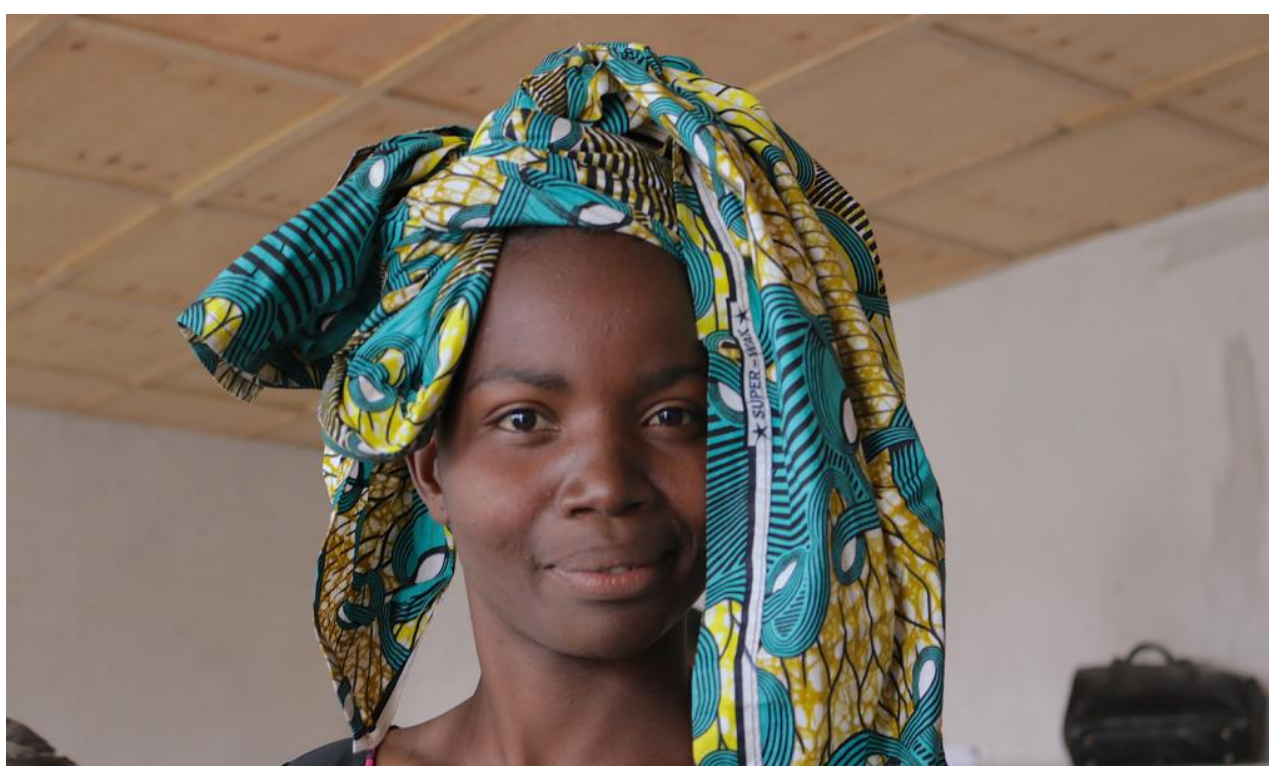

Participant Natalie demonstrating what she can do during the wrapper game activity in Colors of Connection's Tunaweza Portraits Project, Goma, DRC 2019. Photo Credit: Bernadette Vivuya.
Creative Asset 2: Know how to express feelings with and without words Creative Asset 7: Know how to tell a story

Creative Asset 8: Know that she has things in common with others in her community

Source: Christina Mallie, Colors of Connection, www.colorsofconnection.org

adapted from Kenyan performance scholar Mshai Mwangola

https://www.youtube.com/watch?v=3d98MkOHHml

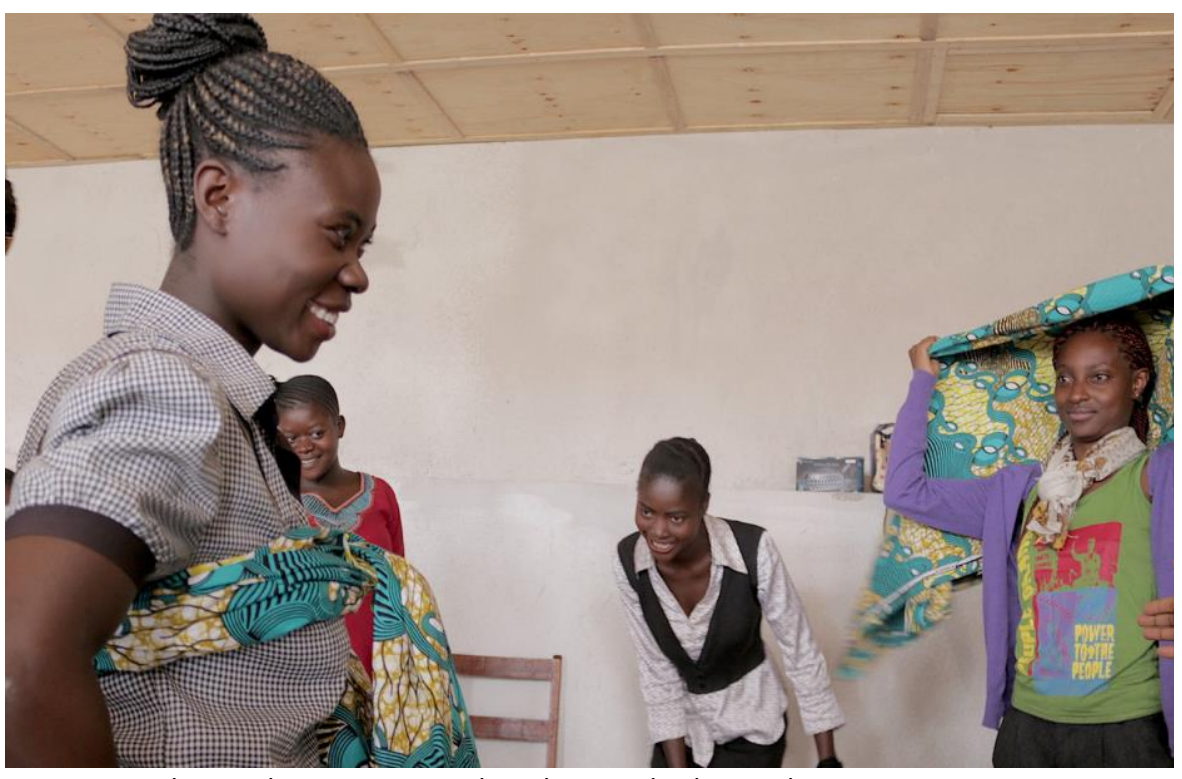

Participant Chance demonstrating what she can do during the wrapper game activity in Colors of Connection's Tunaweza Portraits Project, Goma, DRC 2019. Photo Credit: Bernadette Vivuya. 


\section{Instructions:}

Introduce the activity to the participants by explaining that there are some objects in our lives we use all the time for many different things and one of these is the wrapper. Other names for this may be: lappa, kanga, kikwembe, or payne. Ask participants what their name is for it. Then ask participants to take the following steps:

1. All the participants make a big circle.

2. One at a time, participants go into the middle of the circle without saying any words and demonstrate something that they know how to do with the wrapper. It is possible to sing a song or dance, or perform a theatrical skit, the only rule is that you don't explain what you are doing with words and that you don't do that same thing as another else.

3. Repeat the activity until everyone has participated.

4. Close with reflection of whether participants learned new things to do with the wrapper. Encourage any reflection on the creativity and knowledge that was expressed by participants, highlighting their capabilities. 


\section{ACTIVITY 31: Story Circle}

DURATION: 30 minutes (variable depending on how many participants are sharing)

Objective: Girls will develop improvisational skills, spontaneity, collaboration and cooperation with others, use their imagination, consider new ideas, enhance their listening skills, and actively participate.
Creative Asset 7: Know how to tell a story

Creative Asset 8: Know that she has things in common with others in her community

Source: Developed by storyteller/author Heather Forest for her storytelling workshops with participants, teachers, and librarians.

http://www. heatherforest.com/

\section{Instructions:}

This story-telling activity can begin with a pre-selected title or subject to guide the improvisation.

1. One participant begins a tale and stops after a few sentences. The next participant picks up the story thread and continues it, then stops.

2. The next participant adds to it and so on until the tale comes to a resolution.

3. Try recording the story circle on a tape recorder for later listening so that the group can hear themselves.

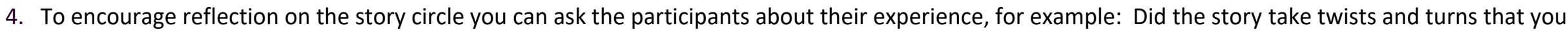

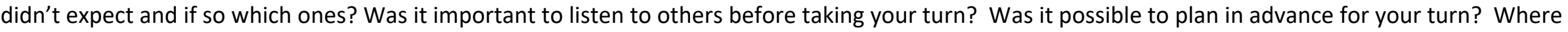

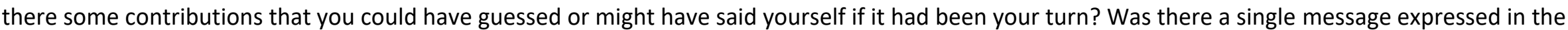
story, like a lesson? 
DURATION: 45 minutes (varies depending on number of participants)

Overview: Participants collect historical stories from their community.

Objective: Girls will learn about their context/culture, society, consider new ideas, develop collaboration and cooperation with others, practice leadership, enhance listening skills, actively participation, and recognize commonalities with others.

\section{Creative Asset 7: Know how to tell a story}

Creative Asset 8: Know that she has things in common with others in her community

Source: Developed by storyteller/author Heather Forest for her storytelling workshops with participants, teachers, and librarians.

http://www.heatherforest.com/

\section{Instructions:}

1. Ask participants to select older people in their community to collect stories from and/or visit a local historical society to see old photographs or artifacts. If participants are interviewing people in their community have them travel in small groups.

2. Examples of questions they can ask are: How were the streets named? Are there any interesting people or legends to which the street names refer? Are there any local places in town about which people tell stories? Any haunted houses? When the town was founded and by whom?

3. These stories are then shared with the whole group of participants and the facilitator.

4. To share these stories more widely, the stories can also be written down and collected into a book of local stories by the participants or facilitator, and/or shared with the community in an "open mic" night in the village where elders come to hear the girls retell these stories. 
DURATION: 45 minutes (varies depending on number of participants)

Overview: This improvisational speech exercise encourages confidence in a participant and their ability to describe a sequence of events. The journey from one's own front door to one's bedroom is well known by the speaker. The speaker may discover in discussing this exercise afterwards, that he or she imagined the house clearly and "saw" more detail than was mentioned. Telling a folktale has a similar process. The teller imagines the landscape of the tale and guides the listeners on a mental journey.
Creative Asset 7: Know how to tell a story

Creative Asset 8: Know that she has things in common with others in her community

Source: Developed by storyteller/author Heather Forest for her storytelling workshops with participants, teachers, and librarians.

http://www.heatherforest.com/

Objective: Participants will develop collaboration and cooperation with others, practice leadership skills, enhance their listening skills, develop improvisational skills and spontaneity and foster connection with others.

\section{Instructions:}

1. Working in pairs consisting of a listener and a speaker, have each set of participants imagine that they are standing outside of the speaker's front door.

2. Have the speaker verbally give the attentive listener an imaginary errand to do. The speaker must carefully explain to his or her partner how to go into the house, travel to the bedroom, and, once there, describe where to find a special treasure somewhere in the room.

3. Have the speaker tell the partner a story about why the thing to be retrieved is special and then have speaker verbally explain how to travel back to the front door to bring the special thing out to where the speaker will be waiting. 


\section{ACTIVITY 34: Chain Sentence}

DURATION: 30 minutes (varies depending on number of participants)

Overview: Teams of two participants orally construct the first sentence of an invented story together.

Objective: Participants will develop improvisational skills, spontaneity, collaboration and cooperation with others. This activity also encourages participants to imagine and enhances their listening skills.

\section{Creative Asset 7: Know how to tell a story}

Creative Asset 8: Know that she has things in common with others in her community

Source: Developed by storyteller/author Heather Forest for her storytelling workshops with participants, teachers, and librarians.

http://www.heatherforest.com/

\section{Instructions:}

1. Break the group up into teams of two. Make the first sentence of: a ghost story, pirate story, love story, mystery, any story, etc. Make the last sentence of a ghost story, pirate story, love story, mystery, any story, etc.

2. To start: To orally make the sentence, each person on each team says one word, and the other team member builds the sentence with another word. The team goes back and forth until they decide to end the sentence. Emphasize that it is important for the participants to trust their ears to recognize conventional grammar. Ask the participants to shape the improvisation by setting the tone of the sentence. 
DURATION: 30 minutes (variable depending on number of participants)

Overview: Describe the history of an object backwards.

Objective: Participants will develop improvisational skills, spontaneity, collaboration and cooperation with others. This activity also encourages participants to imagine and enhances their listening skills.

\section{Creative Asset 7: Know how to tell a story}

Creative Asset 8: Know that she has things in common with others in her community

Source: Developed by storyteller/author Heather Forest for her storytelling workshops with participants, teachers, and librarians.

http://www.heatherforest.com/

\section{Instructions:}

1. Collect an assortment of "things:" For example: Piece of Paper, Shoe, Sneaker, Match, Rubber Band, Paper Clip, Socks

2. Explain to participants that everything has a story! Everything comes, in its elemental origin, from the Earth. Ask the participants to imagine the life story of each of those "things." Describe their history backwards through the personal use, purchase, manufacture, to original natural resources from which it or its components were made. Personify the thing and tell its story like an autobiography.

3. Provide an example of: The tale of a piece of newspaper back to the tree in the forest. The tale of a plastic toy's life, tracing its history back to the oil that became plastic and then back to the prehistoric plants that created the oil.

4. Ask the participants to volunteer to come up with their own examples and share them. Participants can come up with their own objects or use objects that are in the room or space that you have collected. 
DURATION: 30 minutes (variable depending on number of participants)

Overview: Thought-provoking proverbs can suggest a larger scenario. This is an opportunity to look at proverbs creatively and imagine the story the proverb suggests.

Objectives: Participants will develop improvisational skills, spontaneity, collaboration and cooperation with others. This activity also encourages participants to imagine and enhances their listening skills. Participants will gain insights into different traditions and values universal life experiences, understand how wisdom is common to all peoples/all cultures, and observe differences and commonalties of individuals communities, and cultures around the world.

\section{Instructions:}

1. Ask participants to choose a familiar proverb and develop a story that can surround and carry that thought. Multicultural proverbs offer interesting insights into the universality of wisdom.

2. You can give the following examples which are some selected proverbs from Wisdom Tales from Around the World by Heather Forest, August House Publishers:

- One finger cannot lift a pebble. (Iranian)

- No matter how full the river, it still wants to grow. (Congolese)

- The laughter of a child lights up the house (Swahili)

- The sieve says to the needle - "You have a hole in your tail" (Pakistan)

- Talk does not cook rice (Chinese)

- If you educate a woman you educate a family (Fanti)
- All monkeys cannot hang on the same branch (Kenyan)

- There are no shortcuts to the top of the palm tree (Cameroonian)

- A fully grown up tree cannot be bent into a walking stick (Kenyan)

- Not all that is black is charcoal (Philippines)

- It is one thing to cackle and another to lay an egg. (Ecuador)
Creative Asset 7: Know how to tell a story

Creative Asset 8: Know that she has things in common with others in her community

Source: Developed by storyteller/author Heather Forest for her storytelling workshops with participants, teachers, and librarians. http://www.heatherforest.com/
- Every kind of animal can be tamed, but not the tongue of man (Philippines)

- The chameleon looks in all directions before moving (Ugandan)

- One dog barks because it sees something; a hundred dogs bark because they heard the first dog bark (Chinese)
- The jungle is stronger than the elephant (South African)

- A crooked branch has a crooked shadow (Japanese)

- A flea can trouble a lion more than a lion can trouble a flea (Kenyan)

- The more feathers a chicken has the bigger it looks (Zambian)

- The roaring lion kills no prey (Nigerian) 


\section{ACTIVITY 37: Painting with Love}

DURATION: 5 minutes

Overview: A group exercise of appreciation to be practiced regularly at the close of a session.

Objectives: Girls practice giving and receiving love.

\section{Instructions:}

At the close of a session or class gather the group into a circle. One participant is chosen to be "Girl of the Day." She goes into the middle of the circle. Instruct:

1. Encircle her and to begin dipping imaginary paint brushes into imaginary cans of golden colored paint.

2. Make long sweeping brush strokes about 6 inches from the "Girl of the Day."

3. The "Girl of the Day" has her eyes closed for this.

4. The rest of the group showers her with affirmation, good wishes, and appreciation. For example, "Thanks for being such a good friend" or "I like playing with you" or "We like having you in the group."

5. Every day a different girl is selected to be the "Girl of the Day."
Creative Asset 13: Be able to identify what beauty means to her

Source: 100 Ways to Build Self-Esteem and Teach Values by Diana Loomans https://www.dianaloomans.com/child.htm with Julia Loomans 


\section{ACTIVITY 38: Tree Pose}

DURATION: 20 minutes

MATERIALS: 5-10 photographs of a variety of types of trees (see examples). It is helpful to have copies of this set of 5-10 photographs so that each group can have their own set.

TO PREPARE: Collect 5-10 photographs of a variety of trees.

Objectives: Girls practice embodying different feelings and communicating these to others.

\section{Instructions:}

1. Introduce the concept of the secret life of trees to the group: Trees are some of the oldest living organisms on earth. Even though they are silent, they seem to communicate different emotions, and thoughts and moods. When we look at the photographs that will be passed around try to imagine the trees as living beings, and then imagine what kind of character they would be, and what they might say to you.

2. Ask participants to form groups of 4-6.

3. Instruct the group: Each participant in each group will take a turn creating a physical gesture that looks like one of the trees in the photographs and the rest of the group has to guess which tree they resemble. Remind the participants that when they choose one to imitate to imagine the tree as an alive character. Remind the participants that whoever's turn it is has to merely look at the tree photo to choose it but should be careful not indicate to the others in the group which one she is looking at.

4. Distribute a set of 5-10 photographs of trees to each group.

5. Ask each group to form a semi-circle or circle with the images of trees in the center.

6. Instruct the groups to have each participant take a turn.
Creative Asset 2: Know how to express feelings with and without words

Source: Adapted from the Healing Arts Toolkit Warm-Ups: First Aid Arts https://www.firstaidarts.org/

Overview: Form groups of 4 to 6 participants, taking turns to create a physical gesture that looks like one of the trees in the photographs. The rest of the group has to guess which tree they resemble.

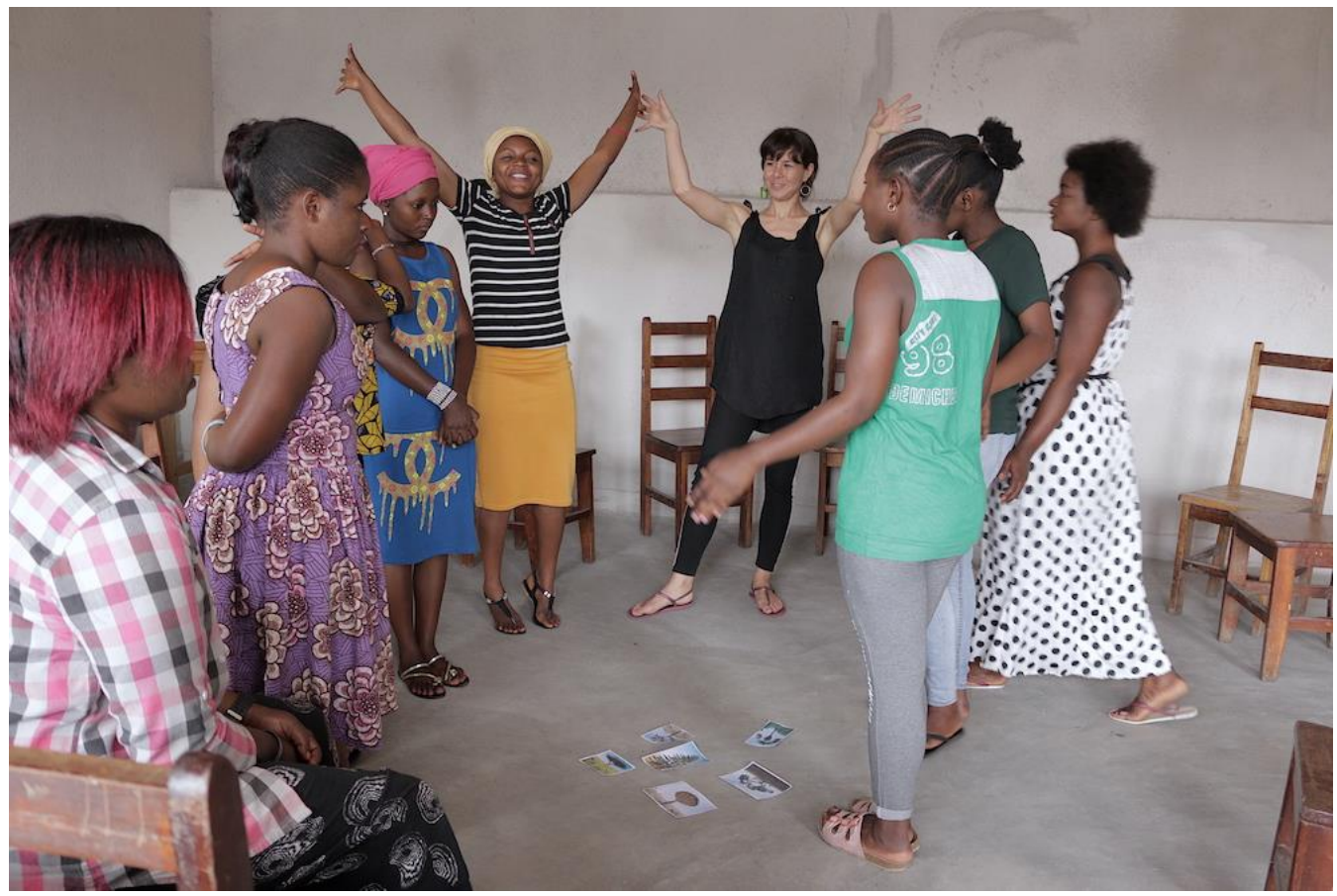

Participants practicing the tree pose with photos of different trees during Colors of Connection's Tunaweza Portraits Project, Goma, DRC 2019. Photo Credit: Bernadette Vivuya. 
7. Discussion about the activity can include asking participants: What was your favorite tree? Can you describe what trees had what emotions, thoughts or moods? Was it difficult or easy to express things with your body without talking? Do you feel differently after the activity and if so in what way?

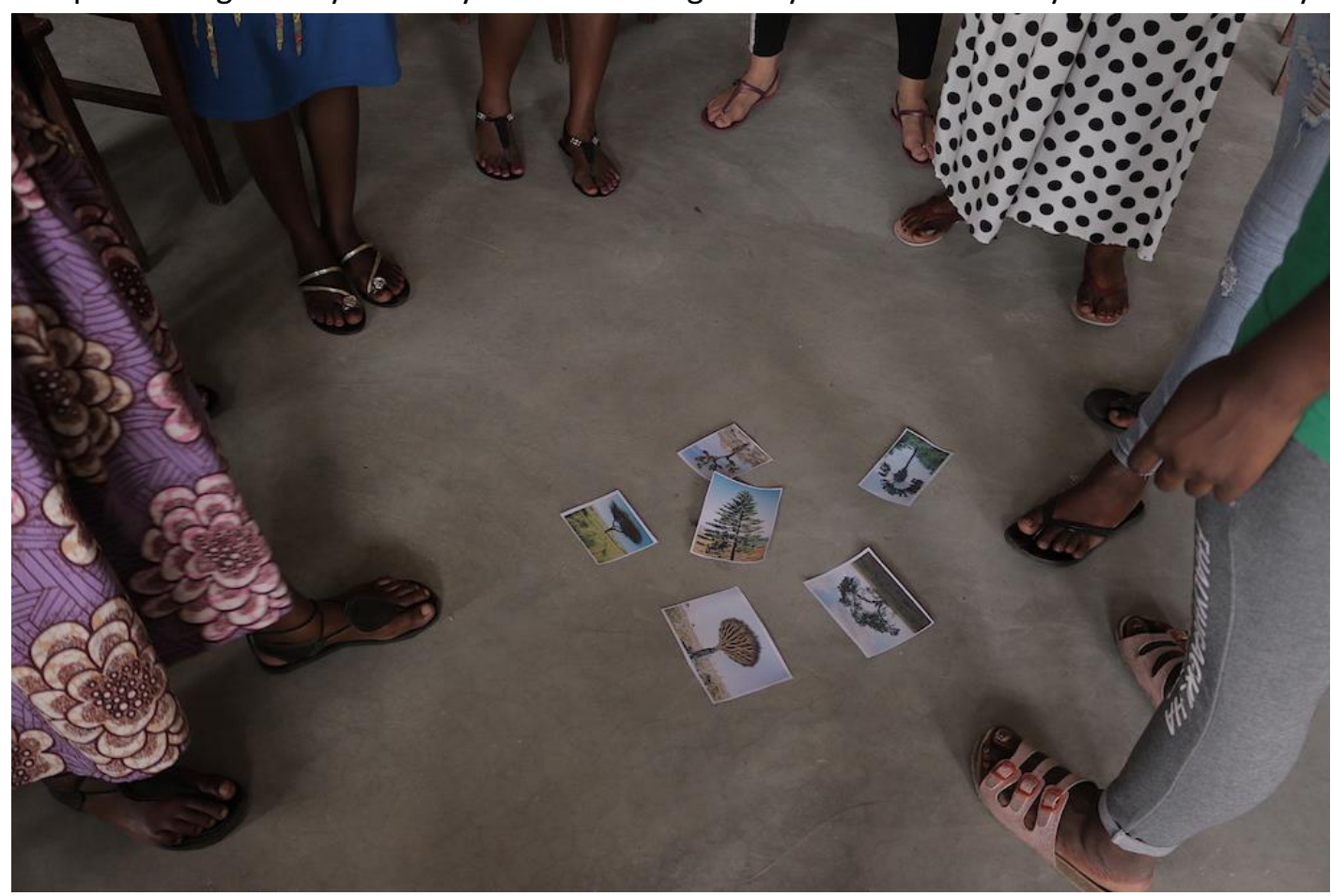

Participants observing different photos of trees before choosing one to imitate during Colors of Connection's Tunaweza Portraits Project, Goma, DRC 2019. Photo Credit: Bernadette Vivuya. 


\section{ACTIVITY 39: High Power Poses}

DURATION: 5 minutes

MATERIALS: Photos of different high and low power poses.

TO PREPARE: Gain familiarity with the various high and low power poses.

See examples of power poses in this article https://iamesclear.com/bodylanguage-how-to-be-confident

and download different photo of examples that will be relatable to participants
Creative Asset 11: Know how to occupy and hold space physically, and psychologically

Source: Amy Cuddy, https://www.amycuddy.com/

Ted Talk: Your Body Language Shapes Who You Are https://www.youtube.com/watch?v=Ks- Mh1QhMc

Overview: Participants practice taking high power poses to boost confidence, energy, and become aware of low power poses.

Objectives: Participants will increase confidence and awareness of expressing their confidence through their body posture.

Note: Adaptations may be needed depending on the culture or context of the participant group. Review the examples of the power poses shared by Amy Cuddy keeping in mind that some poses may be interpreted differently according to the context and culture.

\section{Instructions:}

1. Explain to participants that the way they position their body can affect the way they feel and that it can also affect how others interact with them.

2. Link this to a conversation about safety and self-protection. When are instances in their daily lives that participants may need to feel confident in their bodies? If this is difficult for participants to think of examples you can ask them to tell you what different activities they do on a normal day and find examples in their daily routines, such as taking public transportation, fetching water, or selling produce at the market.

3. Experiment with the participants in taking high and low power poses. Engage participants in a discussion by asking them to share how they feel in the different poses.

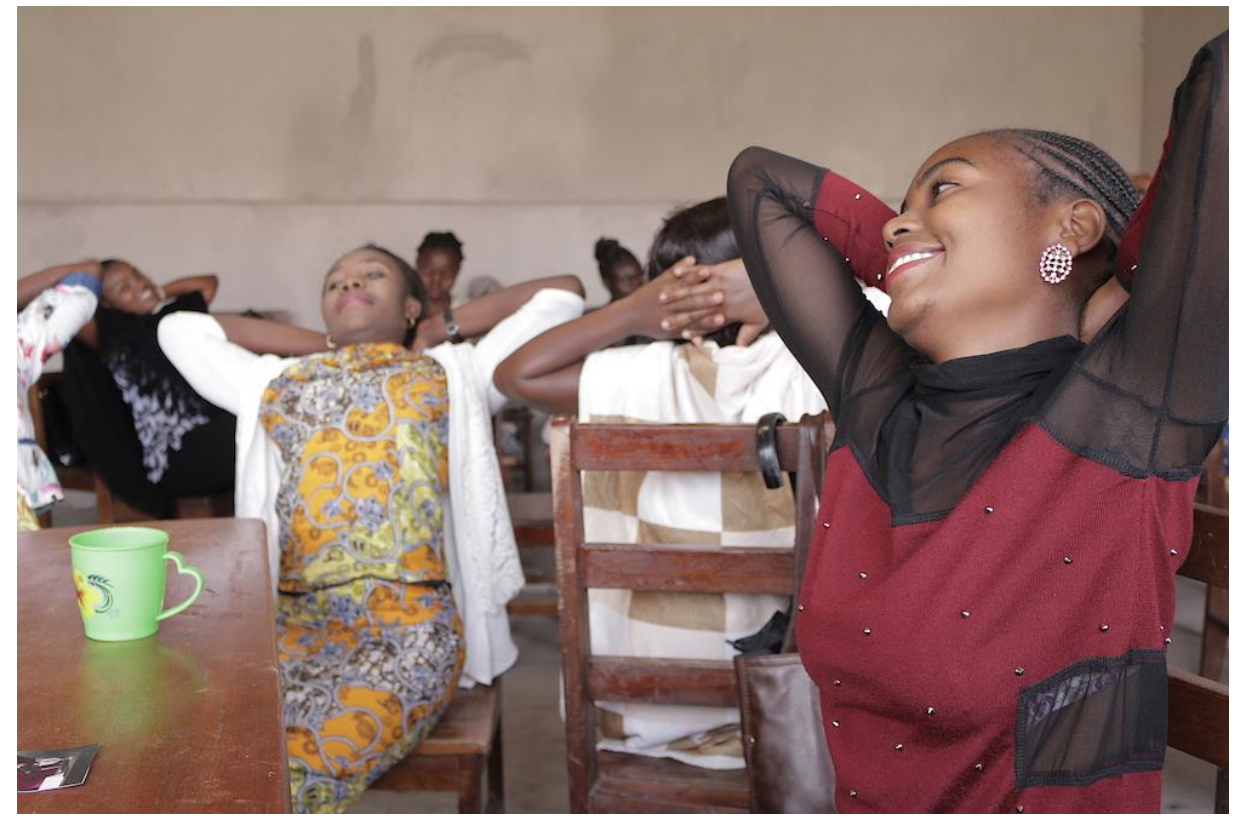

Participants practicing a power pose during Colors of Connection's Tunaweza Portraits Project Goma, DRC 2019. Photo Credit: Bernadette Vivuya. 


\section{ACTIVITY 40: Raising Your Voice and Expanding your Body with Musical Chairs}

\section{DURATION: 15 minutes}

MATERIALS: Music that can easily be turned on and off

TO PREPARE: Arrange chairs or seats in a circle with one less chair than the total group number.

Overview: Participants practice raising their voices from low to high volume and making small to large body movements. The combination of movement with sound integrates the experiences into the body. The musical chairs aspect brings an aspect of spontaneity that relieves stress, self-consciousness, and helps participants access other unknown parts of themselves. Girls then share their experiences of engaging with the activity at the end with the group.

\section{Instructions:}

1. Explain to participants that having a loud voice and expansive body language is a way to express and feel confident.

2. Using the musical chairs model, the person who is left standing once the music stops responds to the following directives. Give several people and/or everyone in the group a chance to respond to the same directive before moving onto the next one. Tell them that they can use real sounds from life for example sounds you hear in the street

\section{Directives:}

a. Make the quietest sound you can with a corresponding body movement

b. Make a medium-sized sound with a corresponding body movement

c. Make a loud sound with a corresponding body movement

3. Open it up for discussion: ask the participants what it was like to make the different sounds, if it felt different in their bodies.
Creative Asset 8: Know that she has things in common with others in her community

Creative Asset 10: Know how to establish a safe space for a group

Creative Asset 11: Know how to occupy and hold space physically, and psychologically

Source: Bonnie Hirschhorn, LCSW, LCAT, ATR-BC, NCPsyA

http://www.bonniehirschhorn.com/

Objectives: Participants will experience a form of taking up space through their voices and bodies, become aware of ways to express themselves with confidence, and build a sense of solidarity.

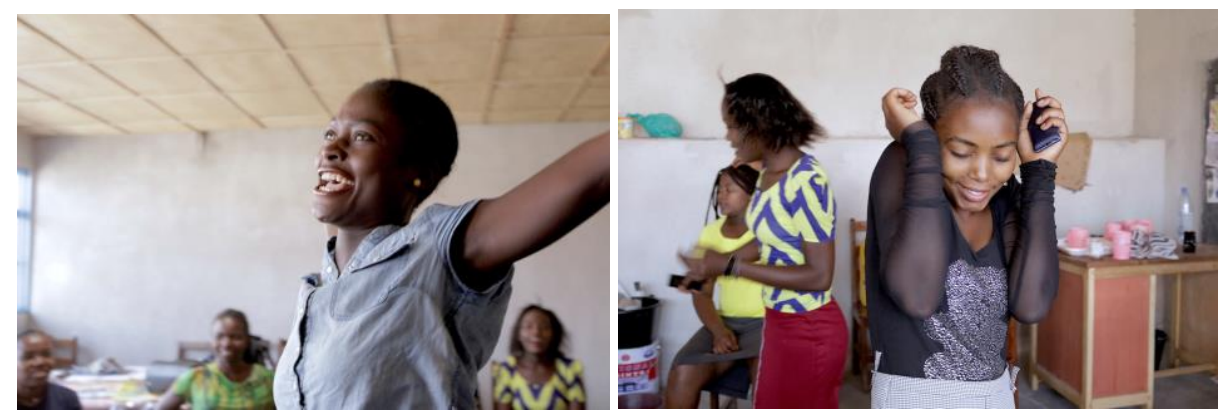

Participants engaging in small and large sounds and gestures for the activity during Colors of Connection's Tunaweza Portraits Project, Goma, DRC 2019. Photo Credit: Bernadette Vivuya. 


\section{ACTIVITY 41: Warm Up}

DURATION: 10 minutes

MATERIALS: Upbeat music that the participants like is recommended but not required.

Overview: This is a group activity in which participants dance the same movement combination together.

Objectives: Participants will build awareness of their bodies and the surrounding environment. Participants will build boundaries and practice respecting others' movements and boundaries.

\section{Instructions:}

Instruct participants in the following demonstrating the movement first:

3. Standing with your legs wider than your shoulders and your feet facing forward, inhale, arms up through the side to above your head, and exhale, bend legs, bring arms down and bend forward (x8)

4. Standing with your legs wider than your shoulders and your feet facing forward, put your hands on the floor and do quick knee bends (x8)

5. Standing with your legs wider than your shoulders and your feet facing forward, put your hands on the floor, lift your heels up and down ( $x 8$ )

6. Standing with your legs wider than your shoulders, right to left arm swinging motion ( $x 8$ )

7. Same pattern is repeated six times, four times, two times, then once.
Creative Asset 9: Know how to establish a safe space for her to be creative

Creative Asset 11: Know how to occupy, hold and defend space physically, socially and psychologically

Source: MindLeaps https://mindleaps.org/ 


\section{ACTIVITY 42: Opposites Improvised Movement}

DURATION: $10-15$ minutes

MATERIALS: Using music is recommended, paper and pencils or pens.

Overview: This is a group activity using language and movement.

Objectives: Participants will recognize their own emotions and the emotions of others. Participants will gain the skills to build group relationships.
Creative Asset 2: Know how to express feelings with and without words Creative Asset 8: Know that she has things in common with others in her community

\section{Source: MindLeaps https://mindleaps.org/}

Note: This exercise can be adapted to illiterate populations by having the facilitator write everything down.

\section{Instructions:}

1. As a group, write down a list of simple opposites (e.g.: "up and down," "sharp and smooth," "small and large.")

2. Say one word out loud. Let participants improvise across the floor with a movement related to that word. Continue this pattern until all words have been used.

3. Assign one word to each participant. For now, have participants keep their word silent.

4. Participant should create 4 counts of movement that describes the word. For example, if a participant's word is "up," her 4 counts of movement should all be up. She should borrow ideas from the "up" improvisation exercise.

5. Next, each participant shows their movements and the group tries to guess what word the movement is describing. Continue this pattern until all participants have shown their movements.

6. Tell participants to find the person with their opposite word. 


\section{ACTIVITY 43: Yoga Balancing Challenge}

DURATION: 15 minutes, modifying to shorter or longer periods as desired.

TO PREPARE: Practice yoga poses: tree pose, warrior , handstand hops, dancer's pose, and side plank.

Objectives: Participants will learn to take risks safely and embrace their mistakes as part of a learning process.

\section{Instructions:}

1. Explain to participants: Balancing poses in yoga encourage taking risks and embracing mistakes as part of a learning process.

2. Choose any of the following poses to practice with participants: Tree pose, warrior 3, handstand hops, dancer's pose, and side-plank. These poses can be made more challenging if already mastered by asking participants to close their eyes.

3. Explain to participants that is it okay to experience moments of imbalance, encourage laughter, and to try the pose again when balance is lost.
Creative Asset 5: Know that making mistakes is an opportunity to learn

Source: Jordan Ashley, SoulJourn Yoga Foundation

http://www.souljournyoga.com/

Note: Illustrations or photos are needed for this activity. This activity can be done individually or in a group.

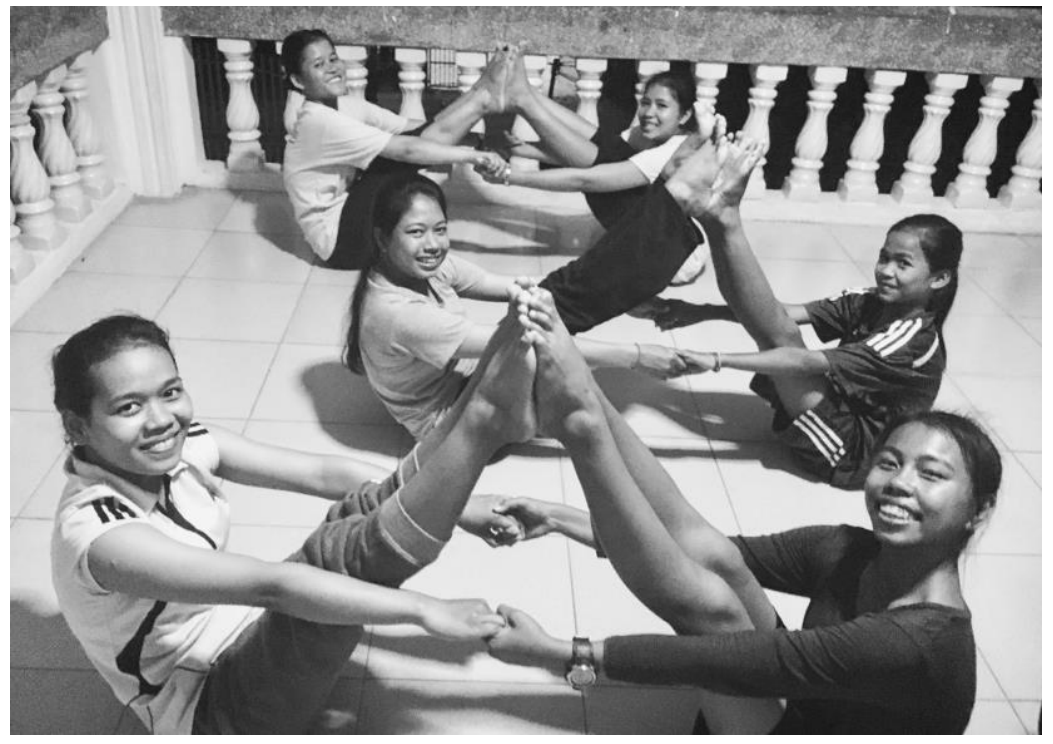

Photo

Yoga creates balance and connection for girls in Cambodia, SoulJourn Yoga Foundation.

Credit: SoulJourn Yoga Foundation Foundation 


\section{ACTIVITY 44: Restorative Yoga Poses}

DURATION: 15 minutes

MATERIALS: Blankets, pillows and blocks

TO PREPARE: Practice yoga poses in activity

Overview: A group exercise of appreciation to be practiced regularly at the close of a session.
Creative Asset 3: Know how to self-soothe

Source: https://greatist.com/fitness/restorative-yoga-infographic

Recommended by yoga instructor Rebecca Pacheco.

Objectives: Participants will be able to calm the mind and body, and reduce stress.

\section{Instructions:}

Lead participants through the following poses. Encourage them to practice these at home in times of stress.

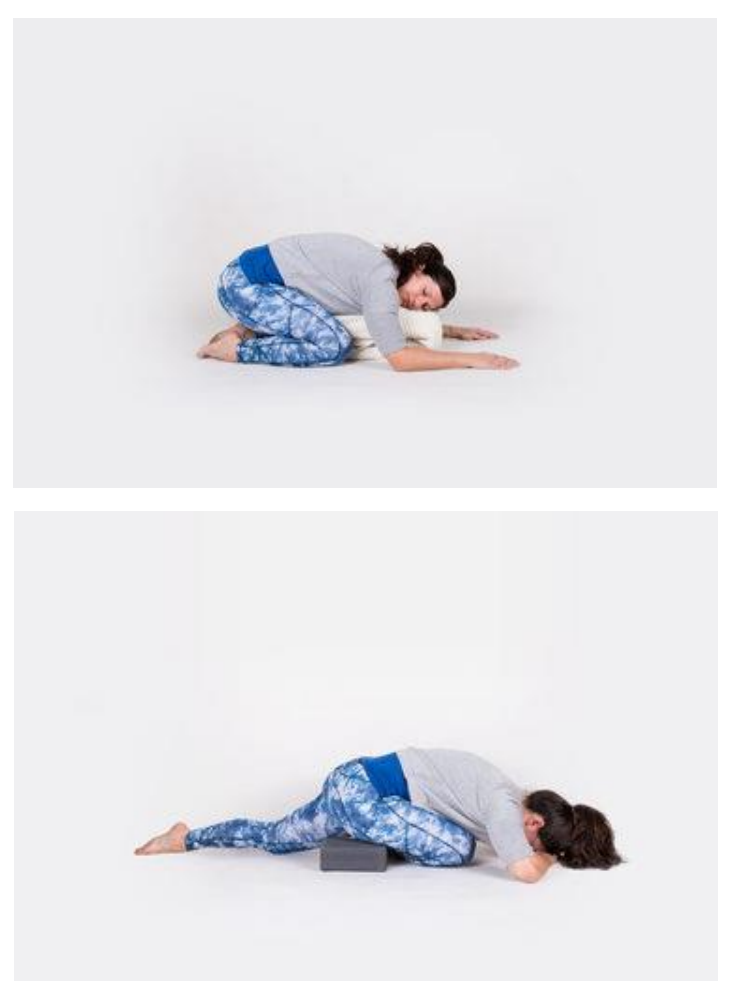

\section{Child's Pose}

Create a support of firm pillows or blankets, lengthwise in front of you. Lower onto your knees, placing them on either side of the support, toes together. Fold your upper body over the length of the support. Rest your forearms on the floor along the support and turn to one side (or rest your forehead on the support-whatever is most comfortable).

Let gravity pull your legs toward the earth as you soften the lower back and release the tailbone toward the heels. Halfway through, turn your head to the other side as the body continues to settle into the pose.

\section{Half-Pigeon Pose}

Start on all fours and slide your right knee forward toward your right hand. Lower your right hip onto a pillow, or folded blanket as you extend your left leg back, toes pointed. Remain upright using hands to support you, or lower down to forearms.

Breathe into the pose as you inch your right shin closer to the mat. Carefully come up to downward-facing dog, then lower onto your left hip to repeat on other side. 

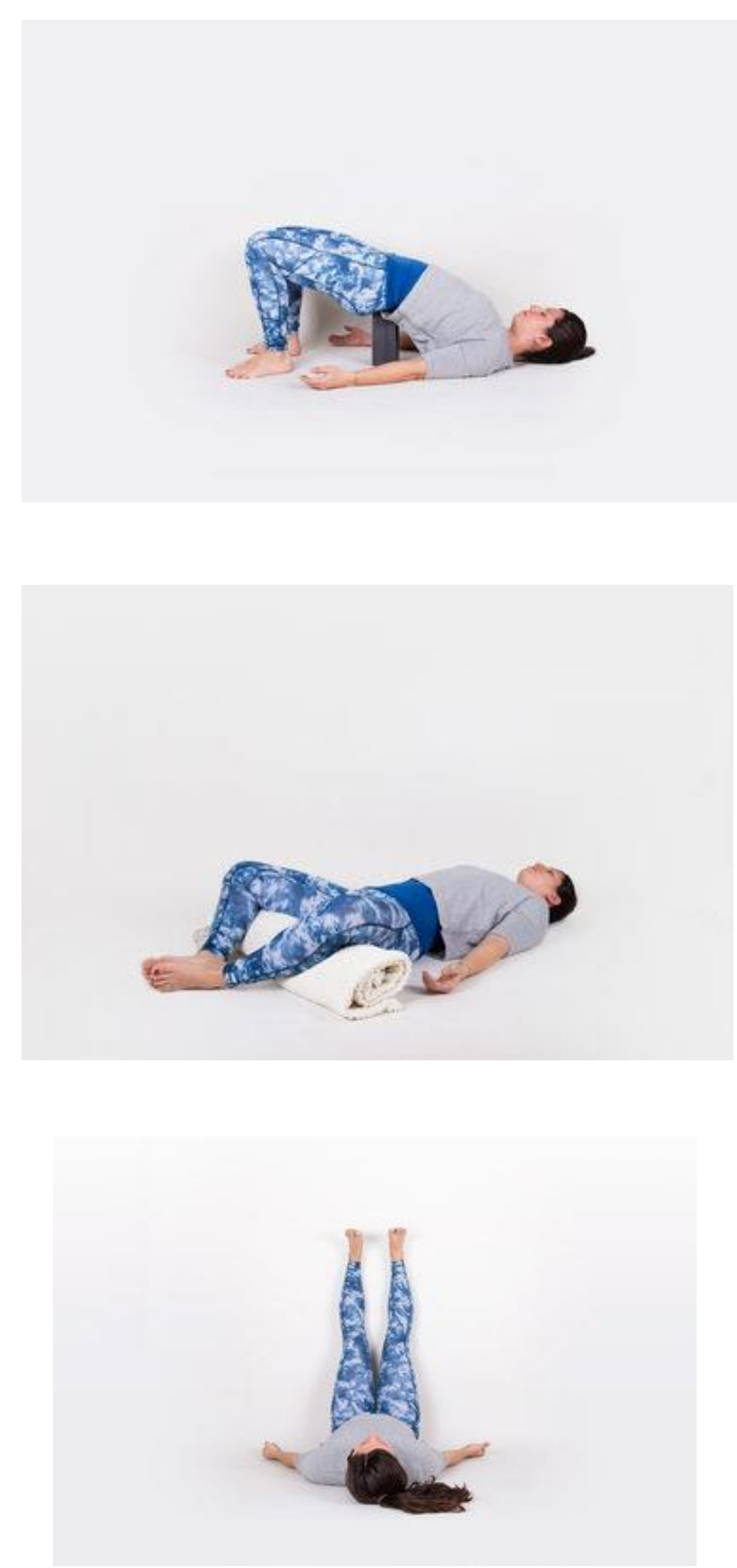

\section{Supported Bridge Pose}

Lie on your back with your knees bent, feet planted flat on the floor, hip-distance apart. Extend your arms by your sides and roll your shoulder blades in toward one another to feel a slight lift in the chest. Carefully lift your hips off the ground and slide a block directly under your sacrum, the large, triangular bone at the base of your spine. (Note: Always come into bridge pose before you place the block under your sacrum - don't try to lie directly on the block.)

As you rest here, arms can remain by your sides, stretched overhead, or straight out in a T-shape. Beginners can keep the block at its lowest height, then rotate it for a higher lift. To come out of the pose, press down into your feet and lift your hips. Remove the block and gently lower back to the floor.

\section{Reclining Bound Angle Pose}

Lie on your back with the soles of your feet together, knees pointing outward, and slide heels as close to the groin as is comfortable. Place your arms at 45-degree angles to your torso with the palms facing up, or place one hand on your low belly and one on your chest to help focus on the breath.

With each exhale, let your knees float toward the ground, creating a stretch across the inner thighs and groin. If the stretch is too deep, place additional blankets or cushions under your knees for support. To come out of the pose, gently roll to your right side with knees drawn to your heart, and gradually prop yourself up to sitting.

\section{Legs-up-the-Wall Pose}

To perform this passive inversion, start seated on the floor with your right shoulder, hip, and thigh against a wall. Keep a bolster within reach, and exhale as you lower your back while swinging your legs up onto the wall. Extend arms out to the sides or in cactus (upper arms perpendicular to torso, elbows bent at 90 degrees, palms facing up). Place a small roll (like a rolled-up towel) under your neck if extra support is needed. Rest passively.

Gently lower your pelvis to the floor, roll to the right side, and use your hand to press yourself back up to sitting. 


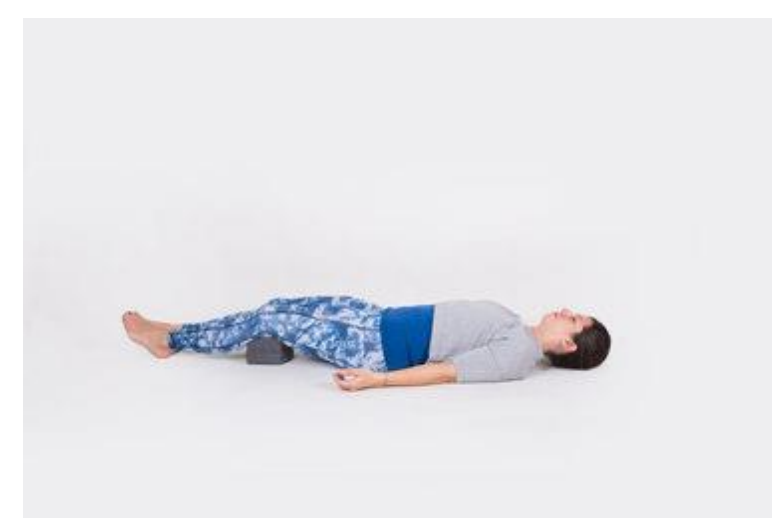

\section{Corpse Pose}

Seated on the floor with legs slightly bent, slide a bolster beneath your knees and slowly lower your back, neck, then head onto the floor. Let your arms rest comfortably by your sides, palms facing the sky. Close your eyes, become aware of your natural breath, and let go. When ready to come out of the pose, roll onto your right side, take 2 to 3 breaths, and gently press your hands into the ground to lift you back to sitting.

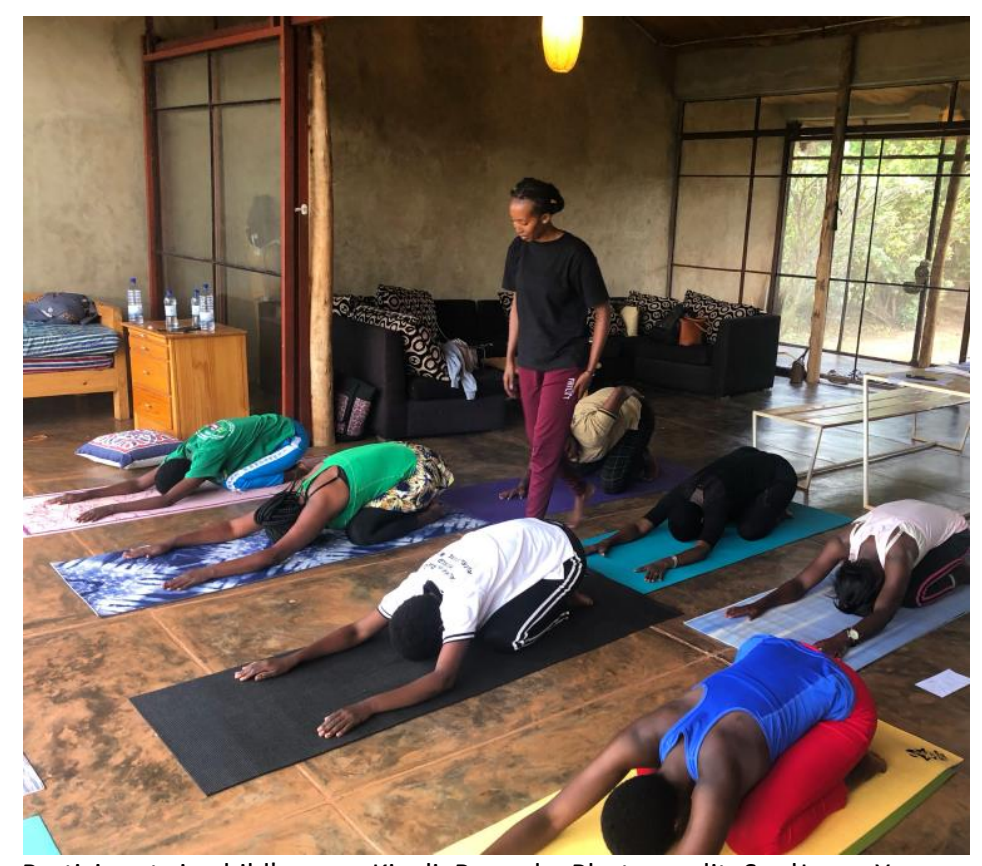

Participants in child's pose, Kigali, Rwanda. Photo credit: SoulJourn Yoga Foundation

Foundation.

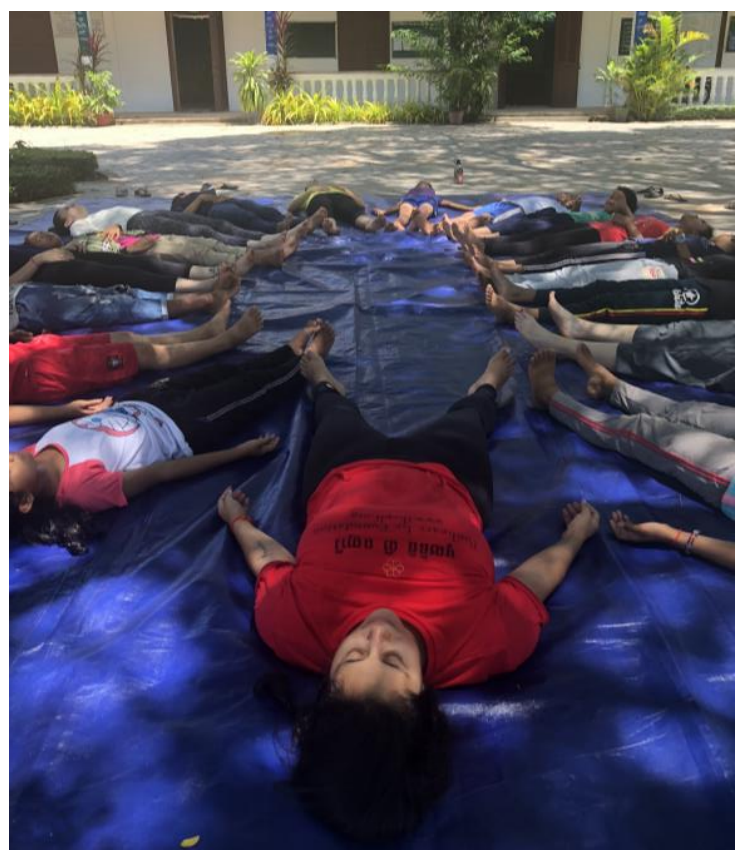

Participants in corpse pose. Photo credit: SoulJourn Yoga Foundation Foundation. 


\section{ACTIVITY 45: Spectrogram Warm-Up}

\section{DURATION: $15 \mathrm{~min}$}

Overview: The group arranges themselves in the room in relationship to each other based on a variety of directions from the facilitator.

Objectives: Participants will experience spontaneity and connection with others, are brought into the present moment, recognize commonalities and differences, and feel more comfortable with themselves and with others. It can also be a tool to help girls identify and clarify issues.
Creative Asset 8: Know that she has things in common with others in her community

Creative Asset 10: Know how to create a safe space for a group

Source: Bonnie Hirschhorn,, LCSW, LCAT, ATR-BC, NCPsyA

http://www.bonniehirschhorn.com/ based on techniques of Jacob L. Moreno.

For more information: http://moreno.com.au/spectrogram-psychodrama

\section{Instructions:}

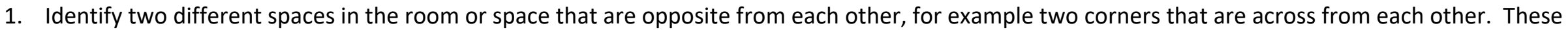
will be the two ends of the spectrum.

2. Explain to the participants that they will arrange themselves along a line between the two chosen points depending on how they respond to the questions. Since all participants will be placing themselves along this line, they will also need to place themselves in relationship to each other.

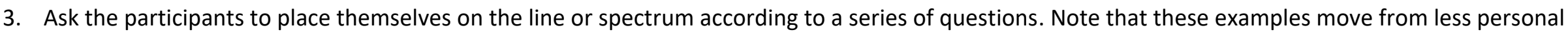

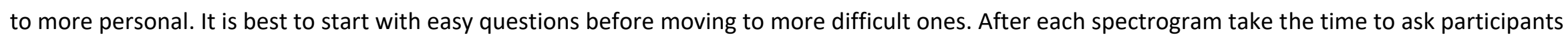
to volunteer to explain why they placed themselves where they did on the line. You can have the group take notice of who is at the extremes, who is in the middle, who learned something new about someone.

The following questions are used to encourage spontaneity and connection with others. To recognize commonalities and differences.

- Where you were born in relationship to this exact place that you are all located today. The person who comes from the furthest away will be on one end of the spectrum and the person who comes from the closest place will be on the other end.

- How long you have lived in this community (0- whomever has lived here the longest).

- How many siblings you have (0- whomever has the most).

- How many years of schooling you have had (0- whomever has the most).

- How close you live to a water pump

- How happy or sad you feel.

- If you feel like part of a group. 
- How uncomfortable or comfortable you feel in this moment.

You can also use this activity to get information on how members of the group feel or are affected by particular themes and issues. It's possible to gage group attitudes and individuals' differences through the spectrogram as well as varying perceptions on issues. For example, you could ask first how safe girls feel, and then ask them to place themselves based on how safe the community thinks they are. This could generate information on the different perspectives of different groups and be a launching point for an in-depth discussion.

4. If this activity is used as a warm-up you can close the activity by asking participants to share new things they learned about each other, things they have in common and ways they are different, if they talked to someone they hadn't talked to before. 


\section{ACTIVITY 46: Exploring Movement in the Kinosphere}

DURATION: 15 minutes

Overview: Exploring personal and public space

Objectives: Participants will experience increased spontaneity, and reduced anxiety, bringing them into the present moment, and developing a sense of their bodies in personal and public space, and connecting with others.
Creative Asset 11: Know how to occupy, hold and defend space physically socially and psychologically

\section{Source: Bonnie Hirschhorn, LCSW, LCAT, ATR-BC, NCPsyA}

http://www.bonniehirschhorn.com/

\section{Instructions:}

1. Ask participants to move around the room in a random fashion. You will begin calling out the directions, giving space between each one for the

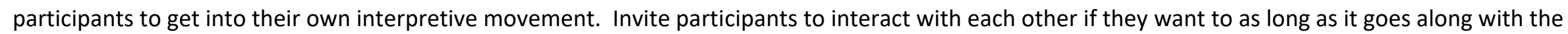

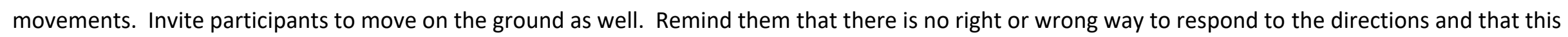
is a silent exercise.

\section{Directions:}

Move as if you were: a worm or starfish.

Freeze.

Move quickly.

Move slowly.

Move as if you were walking in deep water. . . . as if you were in quicksand.. . . as if you were on a hot sandy beach.

Move holding your breath, and then move breathing freely.

Move as if you were in one of the following roles: Outgoing, introverted, an authority, a new student, in kindergarten.

Choose a role you would like to acquire. Move around the room in that role.

Connect with someone else and partner with them. Take turns mirroring each other.

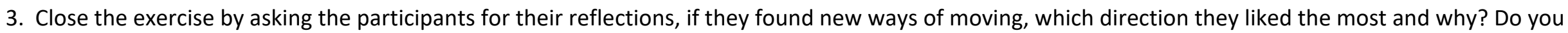
feel different in the space than you did before the activity? If so, what is different? 


\section{ACTIVITY 47: The Circle Game}

\section{DURATION: $10 \mathrm{~min}$}

Overview: Collective group movement in a circle.

Objectives: Participants will foster connection, coordination, and interconnectedness

\section{Instructions:}

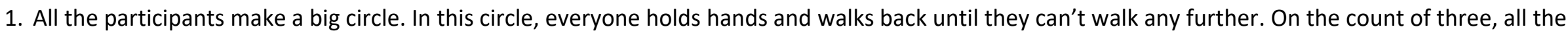
participants must sit on the ground and try to stand back up. They will see that this is very difficult.

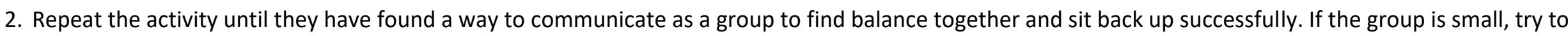
do this with one leg up, or eyes closed.
Creative Asset 10: Know how to establish a safe space for a group

Source: Momentom Collective https://www.momentomcollective.com/ 


\section{ACTIVITY 48: The Shape Game}

DURATION: 30 minutes

TO PREPARE: Review the activity before facilitating to be able to give several examples to the group.

Overview: In this game, participants are asked to create shapes in three different levels.
Creative Asset 2: Know how to express feelings with and without words

Creative Asset 8: Know that she has things in common with others in her community

Source: Momentom Collective https://www.momentomcollective.com/

Objectives: Participants will foster interconnectedness, communication, and personal expression

\section{Instructions:}

1. Divide the group into pairs.

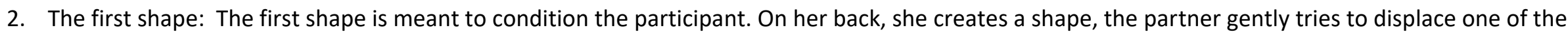
limbs or rock her from side to side, and the one holding the shape is challenged is to stay in the same shape.

3. The second shape: On her feet, one participant stands in mountain pose, also called Tadasana. The partner is instructed to create a statue of her partner by moving the body in whichever way she wants. The pair then showcase their statue to the rest of the group. Then The pairs switch roles.

4. The third shape: Starting again in mountain pose or Tadasana, the one starts moving her body slowly and the partner must mirror her movement. After a few minutes the pairs switch roles.

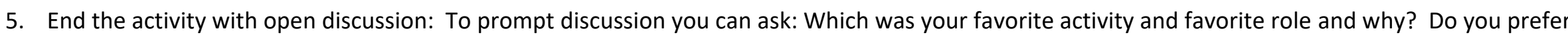
leading or following? Did some of the movements and poses have an emotion or attitude that you can name? 


\section{ACTIVITY 49: The Opposite Game}

\section{DURATION: 15 minutes}

Overview: Participants do the opposite movement that the facilitator calls out.

Objectives: Girls foster listening skills, coordination, perseverance after making mistakes

Instructions:

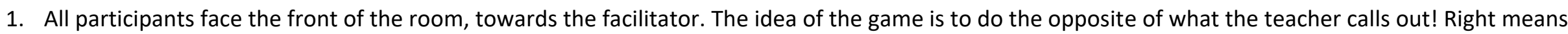

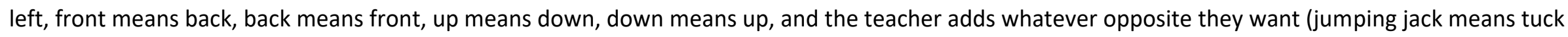
etc.).

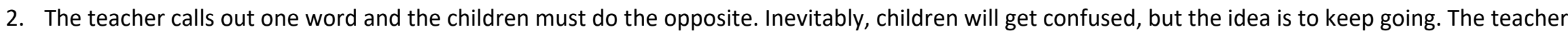
goes faster and faster, and the game becomes more and more fun!

\section{Creative Asset 5: Know that making mistakes is an opportunity to learn}

Source: Momentom Collective https://www.momentomcollective.com/ 


\section{ACTIVITY 50: The Taxi Driver Game}

\section{DURATION: $30 \mathrm{~min}$}

Overview: Working in pairs participants practice guiding each other, with the one being guided having their eyes closed.

Objectives: Girls Build trust, non-visual space awareness, and communication with others.
Creative Asset 4: Know how to gain understanding of herself, her relationships and her experiences

Creative Asset 10: Know how to establish a safe space for a group

Source: Momentom Collective https://www.momentomcollective.com/

\section{Instructions:}

1. Participants are paired in two.

2. Both facing forward, one participant closes her eyes and will be the "taxi" while the other places her hands on her partner's shoulders, arms straight. The participant in the back is the "taxi driver."

3. Without opening her eyes, the participant in front must trust the taxi driver to drive her. In turn, the taxi driver must make sure there is no collision. The facilitator gradually asks the participants to go a bit faster around the room. After a minute or two, the facilitator asks the participants to stop and for the participant in front to keep her eyes closed. The taxi driver than turns her partner a few times in a circle, keeping the eyes closed, and when the facilitator says 'ready', the participant in front makes a T with their arms (either side) keeping their arms engaged. When the facilitator claps, the participant in front must drop backwards and the taxi driver will catch her.

4. Repeat with the partners switching roles.

5. End the activity with an open discussion about what the experience was like. Was it helpful to be able to switch roles? Did it help you understand the other person's experience? Was it difficult or easy to trust your partner when you had your eyes closed? What made it easier or harder to trust your partner? Can any of the things brought up in the discussion be applied to relationships in real life? 


\section{References}

Austrian, K. \& Ghati, D. (2010). Girl Centered Program Design: A Toolkit to Develop, Strengthen and Expand Adolescent Girls Programs. Population Council, 42.

Barndt, D. "Touching minds and hearts: Community arts as collaborative research," Handbook of the Art in Qualitative Research, In Knowles, eds. G. J., \& Cole, A. L., (California: Sage Publications 2008), 351-362.

Bath, H. (2008). The three pillars of trauma-informed care, Reclaiming Children and Youth. 17(3). 18.

Bruce, Judith, Sarah Engebretsen, and Kimberly Glazer. 2015. "Building Assets Toolkit: Developing Positive Benchmarks for Adolescent Girls—Resource Manual." New York: Population Council.

Bruce, J., \& Hallman, K. (2008). Reaching the girls left behind, Gender \& Development. 16 (2). 227-24

Colors of Connection (2016). Courage in Congo Evaluation Report. https://colorsofconnection.org/wp-content/uploads/2019/08/Courage-in-Congo-FinalReport.pdf.

Denborough, D. (2014). Retelling the stories of our lives: everyday narrative therapy to draw inspiration and transform experience. New York, NY: W.W. Norton \& Company, Inc.

Denner, J., Meyer, B., \& Bean, S. (2005). Young women's leadership alliance: youth-adult partnership in an all-female after-school program. Journal of Community Psychology, 33(1) 88.

Eisner, E., “Art and Knowledge," Handbook of the Art in Qualitative Research, eds. In Knowles, G. J., \& Cole, A. L., (California: Sage Publications 2008$), 3-12$.

Emunah, R. (1990). Expression and expansion in adolescence: the significance of creative arts therapy. The Arts in Psychotherapy, 17,101-102.

Feinstein International Center (2017). The impact of mental health and psychosocial support interventions on people affected by humanitarian emergencies, Tufts University. Available at: http://fic.tufts.edu/assets/Mental-Health-Evidence-Brief.pdf.

Greene, M. (1995). Releasing the imagination: Essays on education, the arts and social change. San Francisco: Jossey-Bass Publishers.

Guetzkow, Joshua, "How the Arts Impact Communities: An Introduction to the Literature on Arts Impact Studies," Working Paper Series 20 (2002), (Center for Arts and Cultural Policy Studies, Woodrow Wilson School of Public and International Affairs, New Jersey: Princeton): 3

Hallman, K., Kenworthy, N.J., Diers, J.,Swan N., \& Devnarain, B. (2015). The shrinking world of girls at puberty: violence and gender-divergent access to the public sphere among adolescents in South Africa. Global Public Health, 10(3), 284-291. 
Huss, E., Elhozayel E., \& Marcus E. (2012). "Art in Group Work as an Anchor for Integrating the Micro and Macro Levels of Intervention with Incest Survivors". Clinical Social Work Journal, 40, 401-411.

Kaschak, E. (1992) Engendered lives. New York, NY: Basic Books, 131-148.

Kira, I.A., Ahmed, A., Wasim, F., Mahmoud, V., Coltrain, J., \& Rai, D. (2012). "Group therapy for refugees and torture survivors: treatment model innovations." Int J. Group Psychother 62(1), 69-88.

Johnson, D.R. (1990). Introduction to the special issue on the creative arts therapies with adolescents. The Arts in Psychotherapy, $17,97$.

Lander, D. A., \& Graham-Pole, J. R. (2008). Art as a Determinant of Health. Antigonish, Nova Scotia: National Collaborating Centre, Determinants of Health. Lewis, K.M., Robkin, N., Gaska, K., \& Njoki, C. (2011). Investigating Motivations for Women's Skin Bleaching in Tanzania. Psychology of Women Quarterly, 35(1) 29.

MacNaughton, J., White, M., \& Stacy, R. (2005). “Researching the benefits of arts in health." Health Education, 105(5), $332-339$.

Malchiodi, C.A. (2012). The Healing Arts After a Crisis: 7 Essential Practices. Psychology Today. Retrieved from: https://www.psychologytoday.com/blog/artsand-health/201207/the-healing-arts-after-crisis-7-essential-practices.

Malchiodi, C.A. (2008). Creative interventions with traumatized children. New York, NY: Guilford Press

Malchiodi, C.A. (2007). The Art Therapy Sourcebook. New York, NY: McGraw-Hill. 14-15.

Malchiodi, C.A. (2005). Expressive therapies: history and practice. In C.A. Malchiodi (Ed) Expressive therapies (1-16). New York, NY: The Guildford Press.

Mayer, R. E. (2002). Rote vs. Meaningful Learning. Theory into Practice. 41 (4). 226-233.

McCarthy, K; Brady, M., \& Hallman, K. (2016). Investing when it counts: Reviewing the evidence and charting a course of research and action for very young adolescent. New York: Population Council. 4,7.

Mensch, Barbara S. Erica Soler-Hampejsek, Christine A. Kelly, Paul C. Hewett, Monica J. Grant. 2013. “Challenges in measuring the sequencing of life events among adolescents in Malawi: A cautionary note." Demography. In press.

Mensch, Barbara S., Erica Soler-Hampejsek, Christine Kelly, Paul C. Hewett and Monica J. Grant. 2011. "Transitions to adulthood in rural Malawi: Estimating the sequencing of sexual initiation, school leaving and marriage." Paper presented at the 2011 Annual Meeting of the Population Association of America, Washington DC, 1 April.

Pecora, N., \& Mazzarella, S.R. (2001). Introduction. In S.R. Mazzarella \& N. Pecora (Eds.), Growing up girls: Popular culture and the construction of identity (pp. 121). New York: Peter Lang.

Pennebaker, J.W., \& Seagal, J.D. (1999). Forming a story: the health benefits of narrative. Journal of Clinical Psychology, 55(10) $1243-1252$. 
Perry, B. (2006). Applying Principles of Neurodevelopment to Clinical Work with Maltreated Children. In Webb, N.B. (Ed.), Working with Traumatized Youth in Child Welfare (pp. 27-52). New York: NY: The Guilford Press.

Peterson, J. (1982). The marriage of art therapy and psychodrama. New York, NY: Institute of Sociotherapy Inc.

Professor Bonnie Hirschhorn, personal communication, Oct. 8, 2016.

Schutte, N.S., Malouff, J.M., Simunek, M., McKenley, J., \& Hollander, S. (2002). Characteristic emotional intelligence and emotional well-being, Cognition and Emotion, 16(6), 769-785, DOI: 10.1080/02699930143000482.

Stankiewicz, J.M. \& Rosselli, F. (2008). Women as sex objects and victims in print advertisements. Sex Roles, 58. 579-582.

Steele, C.M. (1997). A threat in the air: how stereotypes shape intellectual identity and performance. American Psychologist, $52(6), 613$.

Women's Refugee Commission (2017, March). Expanding GBV prevention and response programming in Santo Domingo to benefit adolescent refugee girls, Women's Refugee Commission, 4-7. Retrieved from: http://reliefweb.int/sites/reliefweb.int/files/resources/Expanding-GBV-Prevention-Ecuador-March2017.pdf. Woolf, V. (1929). A room of one's own. New York, NY: Harcourt, Inc., 4.

World Health Organization. (2013). Global and regional estimates of violence against women: prevalence and health effects of intimate partner violence and nonpartner sexual violence, 2. Retrieved from: http://apps. who.int/iris/bitstream/10665/85239/1/9789241564625 eng.pdf?ua=1.

World Health Organization. (2014). Global Status Report on Violence Prevention 2014, viii. Retrieved from: http://www.who.int/violence injury prevention/violence/status report/2014/report/report/en/.

Yacine, T. (2001). Women, their space and creativity in Berber society, Race, Gender \& Class. 8(3). 104-106. 
